\title{
Damped Lyman-alpha absorption by disk galaxies with large redshifts. III - Intermediate-resolution spectroscopy
}

\section{Citation}

Turnshek, David A., Arthur M. Wolfe, Kenneth M. Lanzetta, Frank H. Briggs, Ross D. Cohen, Craig B. Foltz, Harding E. Smith, and Belinda J. Wilkes. 1989. “Damped Lyman-Alpha Absorption by Disk Galaxies with Large Redshifts. III - Intermediate-Resolution Spectroscopy." The Astrophysical Journal 344 (September): 567. doi:10.1086/167827.

\section{Published Version}

doi:10.1086/167827

\section{Permanent link}

http://nrs.harvard.edu/urn-3:HUL.InstRepos:30212174

\section{Terms of Use}

This article was downloaded from Harvard University's DASH repository, and is made available under the terms and conditions applicable to Other Posted Material, as set forth at http:// nrs.harvard.edu/urn-3:HUL.InstRepos:dash.current.terms-of-use\#LAA

\section{Share Your Story}

The Harvard community has made this article openly available.

Please share how this access benefits you. Submit a story.

Accessibility 
THE ASTROPHYSICAL JOURNAL, 344:567-596, 1989 September 15

(C) 1989. The American Astronomical Society. All rights reserved. Printed in U.S.A.

\title{
DAMPED LYMAN-ALPHA ABSORPTION BY DISK GALAXIES WITH LARGE REDSHIFTS. III. INTERMEDIATE-RESOLUTION SPECTROSCOPY ${ }^{1}$
}

\author{
David A. TurnsheK, ${ }^{2,3,4}$ Arthur M. Wolfe, ${ }^{3,4,5}$ Kenneth M. Lanzetta, ${ }^{3,4,5}$ Frank H. Briggs, ${ }^{3,5}$ \\ Ross D. Cohen, ${ }^{6}$ Craig B. Foltz, ${ }^{7}$ Harding E. Smith, ${ }^{3,6}$ and Belinda J. Wilkes ${ }^{8}$ \\ Received 1988 August 9; accepted 1989 March 2
}

\begin{abstract}
New intermediate-resolution spectroscopy for certain members of a sample of 68 moderate- to high-redshift QSOs are presented. These new data have been obtained to supplement previously published lower resolution survey data, which we refer to as the Lick survey, in order to identify $\mathrm{H}$ I disk galaxies with large redshifts that give rise to absorption lines in the spectra of background QSOs. Data have been obtained with the MMT and the CTIO $4 \mathrm{~m}$ telescope for six QSOs at 1.0-2.4 $\AA$ resolution. Absorption-line lists are presented, and evidence supporting the identification of seven damped Ly $\alpha$ absorption systems in the six QSOs is discussed. Six of the seven damped Ly $\alpha$ systems have neutral hydrogen column densities $N(\mathrm{H} \mathrm{I}) \geq 2 \times 10^{20}$ $\mathrm{cm}^{-2}$. The damped Ly $\alpha$ systems have absorption properties which include those expected when high-redshift galaxy disks cosmologically intervene along our sight line to QSOs. Therefore, these systems are sometimes termed "Ly $\alpha$ disk" systems. The properties of the damped Ly $\alpha$ systems are discussed and contrasted with other QSO absorption-line systems. About half of the damped Ly $\alpha$ systems would have been missed if they had been searched for on the basis of a spectroscopic survey for C IV absorption alone. Damped Ly $\alpha$ systems are distinguished because they have low ions (i.e., low ionization species) which are generally stronger than the high ions (i.e., high ionization species), they sometimes have other metal line systems clustering around them, and they are the only systems which exhibit $21 \mathrm{~cm}$ absorption. In order to investigate these systems further, a standard curve-of-growth analysis was performed when possible. We suggest that damped Ly $\alpha$ systems arise when a sight line intercepts a high-redshift protogalaxy disk containing a quiescent cloud component characterized by high column density and low effective velocity dispersion. At the same time, the sight line usually intercepts a broader turbulent component, which we identify as the halo, characterized by much lower column density and higher effective velocity dispersion. The turbulent component may contain both low and high ions but about half the time only the low ions are strong enough to be detected. In two of the damped Ly $\alpha$ systems only a quiescent component, with effective velocity dispersion $\sigma<10 \mathrm{~km} \mathrm{~s}^{-1}$, appears to be present. Based on the restrictive assumption that these are single quiescent components, abundances between $4 \times 10^{-3}$ and 1 times solar values are derived. These determinations should be considered strictly lower limits, provided that our metal line identifications and measurements (sometimes in the presence of Ly $\alpha$ forest absorption) are substantially correct. A summary of the incidence of damped Ly $\alpha$ absorption in the Lick survey sample of 68 QSOs is also presented. At least 16 damped Ly $\alpha$ systems with column densities $N(\mathrm{H} \mathrm{I}) \geq 2 \times 10^{20}$ are found over a cosmological path length $\Delta z=56(d N / d z \geq 0.29 \pm 0.07)$ at a mean redshift $z=2.24$.
\end{abstract}

Subject headings: cosmology - galaxies: abundances - galaxies: formation - galaxies: redshifts - quasars

\section{INTRODUCTION}

This is the third in a series of papers describing a survey for dampled Ly $\alpha$ absorption by disk galaxies with large redshifts. Paper I (Wolfe et al. 1986) presented the results of the Lick survey, an unbiased low-resolution ( $\sim 10 \AA$ FWHM) spectroscopic survey of the Ly $\alpha$ forest region in 68 QSOs. The aim of the Lick survey was to identify candidates for galactic $\mathrm{H}$ I disks, i.e., $\mathrm{H}$ I layers with column densities $N(\mathrm{H} \mathrm{I}) \geq 2 \times 10^{20} \mathrm{~cm}^{-2}$.

\footnotetext{
${ }^{1}$ The optical observations presented here were obtained with the Multiple Mirror Telescope (MMT) and the Cerro Tololo Inter-American Observatory (CTIO) $4 \mathrm{~m}$ telescope. The MMT Observatory is a joint facility of the University of Arizona and the Smithsonian Institution; the CTIO is operated by the Association of Universities for Research in Astronomy, Inc. (AURA), under contract with the National Science Foundation.

${ }^{2}$ Space Telescope Science Institute, operated by AURA under contract with the National Aeronautics and Space Administration.

${ }^{3}$ Visiting Astronomer, MMT Observatory.

${ }^{4}$ Visiting Astronomer CTIO, National Optical Astronomy Observatories.

${ }^{5}$ University of Pittsburgh.

${ }^{6}$ University of California, San Diego.

${ }^{7}$ MMT Observatory.

${ }^{8}$ Smithsonian Astrophysical Observatory.
}

This goal was accomplished by searching for the primary spectroscopic signature of an $\mathrm{H}_{\mathrm{I}}$ disk: a $\mathrm{Ly} \alpha$ absorption line broadened by radiation damping to rest-frame equivalent widths of $W(\operatorname{Ly} \alpha) \geq 10 \AA$. Analysis of the Lick data resulted in the selection of 47 strong absorption features which, if they are identified with Ly $\alpha$, have rest-frame equivalent widths $W(\operatorname{Ly} \alpha) \geq 5 \AA$ (the $10 \AA$ minimum absorption equivalent width criterion was relaxed to account for measurement errors) and are distributed over a total redshift path $\Delta z=56$.

The detection of so many strong Ly $\alpha$ absorption features in the low-resolution data was unexpected. Extrapolation of the observed Ly $\alpha$ equivalent width distribution derived for "metal-free" Ly $\alpha$ forest clouds (i.e., the Ly $\alpha$-only population) with $W<3 \AA$ (Sargent et al. 1980, hereafter SYBT) reveals that fewer than $\sim 0.1$ single lines with $W>5 \AA$ should have been found along the redshift path of the Lick survey. Furthermore, extrapolation of the SYBT equivalent width distribution derived at $W<3 \AA$ for the $L y \alpha$ forest clouds containing metals (i.e., the Ly $\alpha$-metal population) suggests that fewer than five lines with $W>5 \AA$ should have been found. Of course, the features need not arise from line broadening by radiation 
damping in a single component, but rather could arise from Doppler broadening, presumably caused by blends of multiple narrow components that span velocity intervals exceeding $\sim 1000 \mathrm{~km} \mathrm{~s}^{-1}$. However, simulations of low-resolution data with the SYBT equivalent width distribution are unable to reproduce more than about one-third of the candidate features (Wolfe et al. 1990, hereafter Paper II $^{9}$ ). Because the spectra of Paper I are inadequate for discriminating damped lines from blends of multiple narrow components, a program of follow-up spectroscopy at higher resolution and signal-to-noise ratio was initiated. While the principal aim was to find damped Ly $\alpha$ lines, another motivation was to determine the nature of all 47 absorption features.

To that end, spectra with improved accuracies for 12 QSOs with candidate disk absorption were acquired. The results for six of these QSOs are presented in this paper; results for the remaining six QSOs are presented in Wolfe, Turnshek, and Lanzetta (1989, hereafter Paper IV). The data set presented here comprises spectra of six QSOs obtained at blue wavelengths with the MMT and spectra of four of these same QSOs obtained at red wavelengths with the CTIO $4 \mathrm{~m}$ telescope. The MMT data were obtained with two goals in mind. The first was to test the damping hypothesis with detailed studies of the Ly $\alpha$ profiles. The second was to detect metal lines at the Ly $\alpha$ redshifts in order to confirm the $\mathrm{Ly} \alpha$ identifications and to survey the metal line properties in order to pave the way for future high-resolution studies of the physical conditions and metal content of the gas. Moreover, comparison between Ly $\alpha$ and metal line velocity profiles provides an independent test of the damping hypothesis. The CTIO data were generally acquired at wavelengths between $\sim 5700$ and $8600 \AA$, in wavelength regions outside the $\mathrm{Ly} \alpha$ forest; the Fe II multiplets and $\mathrm{Mg}$ II absorption doublet are predicted to lie in these unconfused wavelength regions. These data were needed to supplement the MMT spectra because many of the shorter wavelength metal lines are predicted to lie shortward of Ly $\alpha$ emission, where metal lines are difficult to extract due to the confusion noise of the Ly $\alpha$ forest.

Although blending is found to be more extensive than predicted by the simulations, the evidence presented here demonstrates that more than $40 \%$ of the Ly $\alpha$ forest absorption features identified in the Lick survey with $W \geq 5 \AA$ do not arise from Doppler motions, but instead result from quantummechanical radiation damping. These features are generally consistent with having at least one absorption component that is characterized by two main properties which can be inferred from the intermediate-resolution optical spectroscopy alone: (1) large neutral hydrogen column density, e.g., $N\left(\mathrm{H}_{\mathrm{I}}\right) \geq 10^{20}$ $\mathrm{cm}^{-2}$, and (2) low ions which dominate over high ions. ${ }^{10}$ Therefore, such systems have properties which are quite distinct from the QSO absorption-line systems which give rise to the $\mathrm{Ly} \alpha$-only and $\mathrm{Ly} \alpha$-metal populations as discussed by SYBT. (See also Tytler 1987 for a discussion of evidence which may suggest that the $L y \alpha$-metal and $L y \alpha$-only systems arise from a "single population.") Coupled with the quantitative statistical arguments given in Paper II, the observations presented here suggest the presence of a separate population of absorbers that contribute Ly $\alpha$ lines to the Ly $\alpha$ forest (see also Wolfe 1988). Sometimes systems in this population of

\footnotetext{
9 Paper II will be published after Papers III and IV.

10 "Low ions" and "high ions" denote respectively low-ionization species and high-ionization species.
}

absorbers are referred to as "Ly $\alpha$-disk systems," but we shall refer to them as "damped Ly $\alpha$ systems."

On the basis of $21 \mathrm{~cm}$ absorption-line studies of a few of these systems (cf. Briggs 1988 and Paper II) and results from curves of growth presented here, we suggest that a typical damped Ly $\alpha$ system is composed of two components: a quiescent component and a turbulent component. The quiescent component contains most of the $\mathrm{H}$ I which gives rise to damped $\mathrm{Ly} \alpha$ and $21 \mathrm{~cm}$ absorption, has an effective velocity dispersion $\sigma \sim 5-10 \mathrm{~km} \mathrm{~s}^{-1}$, and generally contains only lowionization states of abundant elements. The quiescent component is always present. The turbulent component contains a small fraction of the total $\mathrm{H} \mathrm{I}$, has an effective velocity dispersion of $\sigma>20 \mathrm{~km} \mathrm{~s}^{-1}$, and has multiple components that span a velocity interval $\Delta v_{\text {metals }} \geq 150 \mathrm{~km} \mathrm{~s}^{-1}$; and, while it may contain both low and high ions, the low-ion equivalent widths are larger. In fact, the high ions are not detected in about half the cases (see also Paper IV). The turbulent component may not be present in a small fraction of the damped Ly $\alpha$ systems. The turbulent component bears a resemblance to the "optically thick absorption systems" detected on the basis of Lyman limit absorption or Mg II absorption (cf. Lanzetta, Turnshek, and Wolfe 1987, hereafter LTW; Lanzetta 1988), but the quiescent component is distinct from most optically detected QSO absorbers, resembling instead those QSO absorbers detected in $21 \mathrm{~cm}$ absorption. Paper II elaborates the evidence that the quiescent components are drawn from a unique population of disklike structures that are the highredshift progenitors of galactic disks. The turbulent component may reside in halos which envelop the disks. From curve-of-growth analysis, two cases containing only a quiescent component have been tentatively identified here. Abundance derivations in the quiescent and turbulent components are discussed. Evidence that metal line systems cluster around the damped Ly $\alpha$ redshift is also summarized.

This paper is organized as follows. The new intermediateresolution spectroscopy and absorption-line lists for six QSOs are presented in $\S$ II. In $\S$ III evidence is presented which indicates that seven strong absorption features in the spectra of the six QSOs are due to damped Ly $\alpha$ absorption. A standard curve-of-growth analysis on five of the damped systems is performed, and relevant properties are tabulated and discussed. Six of the seven damped Ly $\alpha$ systems studied have $\mathrm{H}$ I column densities $N(\mathrm{H} \mathrm{I}) \geq 2 \times 10^{20} \mathrm{~cm}^{-2}$, while the remaining system has $N(\mathrm{H} \mathrm{I}) \simeq 10^{20} \mathrm{~cm}^{-2}$. These results are examined in $\S \mathrm{IV}$, where the properties, the differences between the damped Ly $\alpha$ systems and other types of narrow metal line absorption systems, a qualitative model for the absorption, and the derivation of abundances are discussed. The status of candidates for damped Ly $\alpha$ absorption in the Lick survey sample are also reviewed. The conclusions are summarized in $\S \mathrm{V}$.

\section{OBSERVATIONS}

Table 1 summarizes the journal of observations for the six QSOs. This new intermediate-resolution (1.0-2.4 $\AA$ FWHM) optical spectroscopy was obtained because these QSOs were identified as having candidate damped Ly $\alpha$ absorption in Paper I. The wavelength ranges in Table 1 were chosen to include the candidate damped Ly $\alpha$ features and predicted corresponding metal lines. In this section data acquisition, reduction, and analysis procedures which resulted in the absorption-line lists are described. 
TABLE 1

JOURNAL OF OBSERVATIONS

\begin{tabular}{|c|c|c|c|c|c|c|c|}
\hline $\begin{array}{l}\text { Coordinate } \\
\text { Designation }\end{array}$ & $\begin{array}{l}\text { Alternate } \\
\text { Name }\end{array}$ & $z_{\mathrm{em}}$ & Telescope & $\begin{array}{l}\text { UT } \\
\text { Date }\end{array}$ & $\begin{array}{c}\text { Exposure } \\
\text { Time } \\
\text { (minutes) }\end{array}$ & $\begin{array}{l}\text { Wavelength } \\
\text { Region } \\
(\AA)\end{array}$ & $\underset{(\AA}{\text { Resolution }}$ \\
\hline $\mathrm{Q} 0836+113 \ldots \ldots$ & Hazard & 2.67 & $\begin{array}{l}\text { MMT } \\
\text { MMT } \\
\text { MMT } \\
\text { CTIO } 4 \mathrm{~m} \\
\text { CTIO } 4 \mathrm{~m}\end{array}$ & $\begin{array}{l}1986 \text { Mar } 6 \\
1986 \text { Dec } 2 \\
1986 \text { Dec } 3 \\
1987 \text { Feb } 6 \\
1987 \text { Feb } 7\end{array}$ & $\begin{array}{r}80 \\
180 \\
60 \\
100 \\
60\end{array}$ & $\begin{array}{l}3700-5600 \\
3700-5600 \\
3700-5600 \\
7850-8550 \\
7850-8550\end{array}$ & $\begin{array}{l}2.0 \\
2.0 \\
2.0 \\
2.4 \\
2.4\end{array}$ \\
\hline $\mathrm{Q} 1136+122$. & Hazard & 2.90 & $\begin{array}{l}\text { MMT } \\
\text { CTIO } 4 \text { m }\end{array}$ & $\begin{array}{l}1985 \text { Mar } 18 \\
1987 \text { Feb } 8\end{array}$ & $\begin{array}{l}210 \\
180\end{array}$ & $\begin{array}{l}3300-4050 \\
6150-6850\end{array}$ & $\begin{array}{l}1.0 \\
2.4\end{array}$ \\
\hline $\mathrm{Q} 1151+068 \ldots \ldots \ldots \ldots \ldots \ldots \ldots$ & Hazard & 2.76 & $\begin{array}{l}\text { MMT } \\
\text { MMT } \\
\text { MMT } \\
\text { CTIO } 4 \mathrm{~m} \\
\text { CTIO } 4 \mathrm{~m}\end{array}$ & $\begin{array}{l}1984 \text { Jan } 2 \\
1986 \text { Mar } 7 \\
1986 \text { Dec } 3 \\
1987 \text { Feb } 6 \\
1987 \text { Feb } 7\end{array}$ & $\begin{array}{r}100 \\
100 \\
90 \\
140 \\
160\end{array}$ & $\begin{array}{l}3250-4100 \\
3250-4100 \\
4175-5125 \\
6200-6900 \\
7150-7850\end{array}$ & $\begin{array}{l}1.0 \\
1.0 \\
1.0 \\
2.4 \\
2.4\end{array}$ \\
\hline $\mathrm{Q} 1337+113 \ldots \ldots \ldots \ldots \ldots \ldots$ & Hazard & 2.92 & $\begin{array}{l}\text { MMT } \\
\text { MMT } \\
\text { MMT } \\
\text { MMT } \\
\text { CTIO } 4 \mathrm{~m} \\
\text { CTIO } 4 \mathrm{~m} \\
\text { CTIO } 4 \mathrm{~m}\end{array}$ & $\begin{array}{l}1982 \text { Jun } 22 \\
1982 \text { Jun } 23 \\
1982 \text { Jun } 23 \\
1982 \text { Jun } 24 \\
1985 \text { Jun } 16 \\
1985 \text { Jun } 17 \\
1986 \text { Aug } 6\end{array}$ & $\begin{array}{r}60 \\
60 \\
40 \\
100 \\
30 \\
140 \\
80\end{array}$ & $\begin{array}{l}4450-5250 \\
4450-5250 \\
4650-5450 \\
4450-5250 \\
5750-6425 \\
5750-6425 \\
6300-7000\end{array}$ & $\begin{array}{l}2.0 \\
2.0 \\
2.0 \\
2.0 \\
2.4 \\
2.4 \\
2.4\end{array}$ \\
\hline $\mathrm{Q} 1374+112 \ldots \ldots \ldots \ldots \ldots \ldots$ & Hazard & 2.71 & MMT & 1985 Mar 18 & 120 & $4100-5900$ & 2.0 \\
\hline $\mathrm{Q} 2348-011 \ldots \ldots$ & UM 184 & 3.03 & MMT & 1986 Dec 4 & 180 & $3900-5800$ & 2.0 \\
\hline
\end{tabular}

\section{a) Data Acquisition and Reduction Procedures}

Spectroscopy covering blue wavelengths was acquired for the six QSOs with the MMT $4.5 \mathrm{~m}$ and MMT spectrograph at resolutions of $1 \AA$ (FWHM) in two cases and $2 \AA$ (FWHM) in four cases. For the $1 \AA$ resolution data, an 832 line $\mathrm{mm}^{-1}$ grating was used in second order. When near-ultraviolet observations were made, $\mathrm{a} \mathrm{CuSO}_{4}$ filter was employed to block the first-order spectrum. For the $2 \AA$ resolution data, in all but one case an 800 line $\mathrm{mm}^{-1}$ grating was used in first order. For the remaining $2 \AA$ resolution observations (the Q1337+113 observations covering $4450-5450 \AA$ ), a 600 line $\mathrm{mm}^{-1}$ grating was used in second order. For most cases the "image stacker" which incorporates two sets of six 2".5 circular apertures was used. The detector for the MMT spectrograph is an intensified photon-counting Reticon mated to an image intensifier package consisting of a three-stage, magnetically focused ITT image tube directly coupled to a Varo microchannel plate intensifier. In the configuration normally utilized, quarter-pixel substepping allows the detector to collect data into two linear arrays that are effectively 4096 pixels each, one for the objectplus-sky spectrum and one for the sky spectrum $36^{\prime \prime}$ away. This configuration yields 4 pixels per resolution element. The data were obtained in the standard way, moving the object back and forth between apertures every 15 or 20 minutes so that accurate sky subtraction could be achieved. Flux standards were not usually observed. The data were divided by a high signal-to-noise normalized spectrum of an internal quartz lamp, obtained during the day in order to remove any highfrequency variations in sensitivity. Because of the use of an image tube, which introduces spatial distortions, and because of flexure in the spectrograph, which can cause the spectrum to drift systematically in wavelength by as much as 1 resolution element every $1.5 \mathrm{hr}$, care had to be taken in performing the wavelength calibrations. Wavelength calibrations are ideally accomplished using exposures of a $\mathrm{He}-\mathrm{Ar}-\mathrm{Hg}-\mathrm{Cd}$ comparison lamp and a seventh-order polynomial to map the pixel-towavelength transformation. However, in many instances the Cd lamp was not available and the calibrations of the nearultraviolet wavelength portions of certain spectra were uncertain. When necessary, long exposures of the comparison lamp were taken during the day in order to ensure reasonable signalto-noise ratio in the near-ultraviolet parts of certain spectra. In addition, comparison lamp spectra were taken frequently during the course of observations for the purpose of both performing the wavelength calibration and tracking the drift in the calibration due to spectrograph flexure. Usually, observations of an object spanning the course of an hour or more had to be shifted in pixel space before performing object addition and sky subtraction so that the resolution could be maintained. Typically, errors in the wavelength calibrations had rms residuals of about $20 \%$ of a resolution element, but the errors are sometimes worse than this near the ends of the spectra. After the spectra were reduced in this manner, they were resampled onto a linear wavelength scale at intervals corresponding to one-quarter of the resolution element so that they could be co-added with other spectra, in accordance with the signal-to-noise ratio of the data, before analysis. Finally, in all but one case the data were placed on a relative $F_{\lambda}$ flux scale using the spectrophotometry of Lanzetta et al. (1988). The data were placed on a flux scale primarily for the purpose of display, rather than to facilitate any type of analysis.

Spectroscopy covering red wavelengths was acquired for four of the six QSOs with the CTIO $4 \mathrm{~m}$ telescope, the RitcheyChrétien spectrograph and a GEC CCD detector. A 790 line $\mathrm{mm}^{-1}$ grating blazed at $8500 \AA$ (designated KPGLD) was used in first order with a 1.5 wide slit in order to obtain a spectral resolution of $2.4 \AA$ (FWHM). When necessary, an RG610 filter was employed to block the second-order spectrum. The CCD detector is two-dimensional, and a long slit was used. The telescope was guided so that the object remained approx- 
imately at a single position on the slit. The CCD was configured for on-chip binning of 3 pixels to 1 perpendicular to the dispersion; this allowed the spectrum of the object to be confined to roughly two columns and effectively reduced the readout noise. The slit width used guaranteed a resolution of 2 pixels, providing adequate sampling along the dispersion direction. A preflash was used to minimize charge transfer effects, and this resulted in increasing the noise due to the background. The objects were each observed in several exposures of typically 20 minutes duration, and standard stars were observed at intervals throughout the course of the observations for the purpose of later removing telluric absorption features. The data were bias-subtracted and flat-fielded in the usual manner; the flat fields were normalized spectra of a white screen on the dome illuminated by a quartz lamp. A onedimensional spectrum for each object was extracted from each two-dimensional CCD image using an optimal extraction algorithm similar to the one discussed by Horne (1986). Wavelength calibrations were carried out using exposures of a $\mathrm{He}-\mathrm{Ar}-\mathrm{Ne}$ comparison lamp taken throughout the night and a quadratic polynomial to map the pixel-to-wavelength transformation. The rms residuals in the wavelength calibration were always less than $0.2 \AA$, and corrections for wavelength shifts due to spectrograph flexure during the course of an observation were never necessary. Telluric absorption features were removed by dividing the spectra by standard star spectra for which the continuum level had been normalized to unity; this procedure worked well for the majority of the cases, although in a few instances the strong telluric $\mathrm{O}_{2}$ band near $7600 \AA$ was poorly corrected for. After the spectra were reduced, they were resampled onto a linear wavelength scale at intervals corresponding to one-half of the resolution element $(1.2 \AA)$ so that they could be co-added with other spectra according to signal-to-noise ratio before analysis. The small
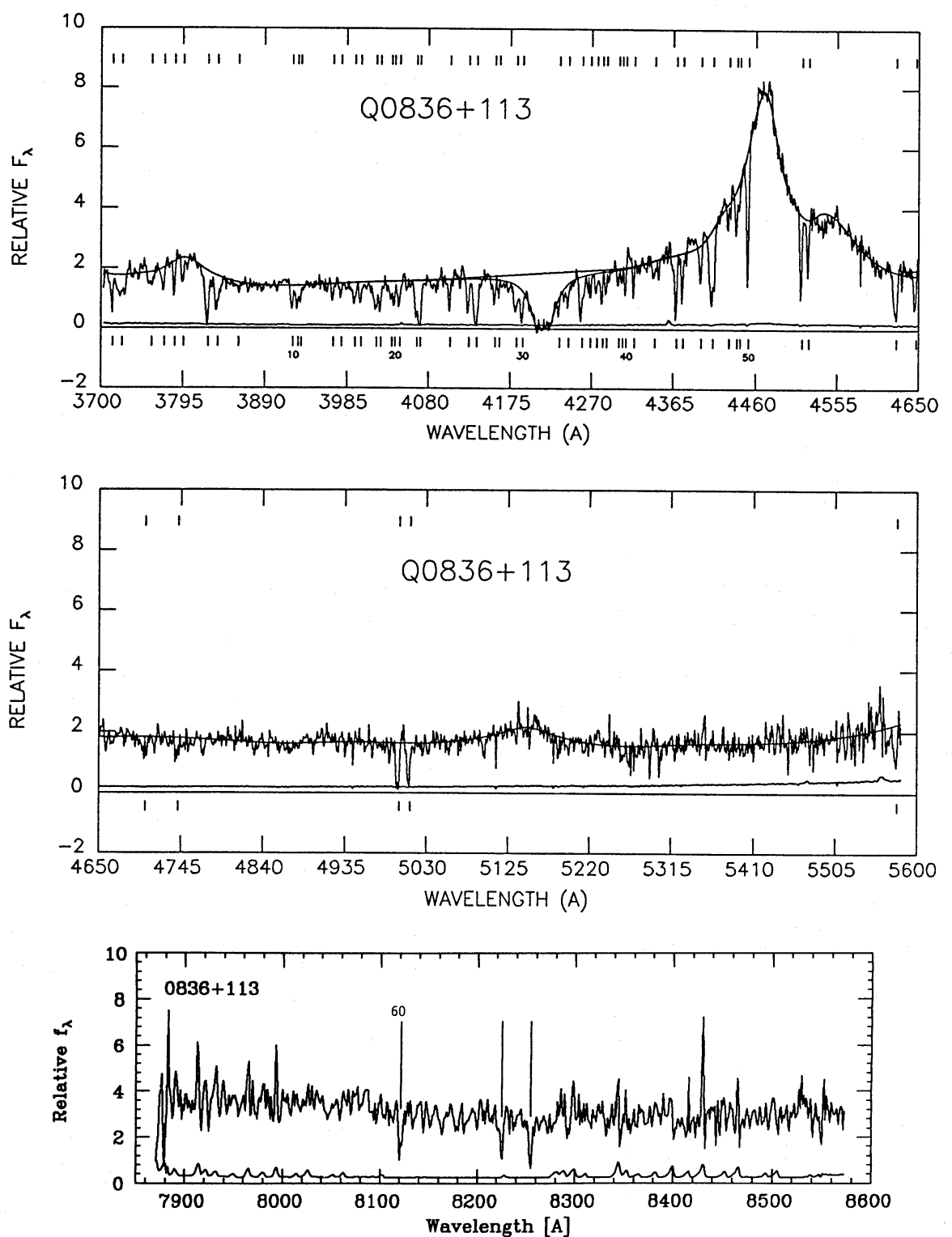

FIG. 1.-Spectrum of Q0836+113 obtained at $2.0 \AA$ resolution with the MMT $(\lambda<5600 \AA)$ and at $2.4 \AA$ resolution with the CTIO $4 \mathrm{~m}$ telescope $(\lambda>7850 \AA)$. The spectrum is unsmoothed, plotted with 2 pixels per resolution element. The $1 \sigma$ error array per pixel in the flux, the adopted continuum, and the fit to the damped Ly $\alpha$ profile are also shown. 
slit that was used rules out spectrophotometry, so the data were not corrected for atmospheric extinction, nor were they flux-calibrated. In any case, the flat response of the detector over the wavelength range covered makes this step unnecessary even for the purpose of displaying the data.

The spectroscopic observations are displayed in Figures 1-6. The data represent approximately $38 \mathrm{hr}$ of on-object exposure, some of which occurred during inclement weather. The data illustrated in the figures are presented unsmoothed, but rebinned at 2 pixels per resolution element. Note that analysis of the MMT data was performed at 4 pixels per resolution element. In all cases, the error in flux, which is also shown in each figure, was determined from photon-counting statistics. In the case of the CTIO $4 \mathrm{~m}$ CCD data, the error in the background, which included the CCD readout noise and the noise produced by preflashing the $\mathrm{CCD}$ detector, was taken into account. For both the MMT and the CTIO data the observations used to flat-field the data had sufficient accuracy so that this error could be neglected.

\section{b) Absorption-Line Lists}

Since the number of photons detected is known, a technique similar to the one employed by Young et al. (1979), and more recently utilized by LTW, was used to determine the absorption-line lists. However, the spectral regions studied in these six QSOs vary considerably in signal-to-noise ratio, and therefore caution should be exercised when using this sample, which is biased for including strong Ly $\alpha$ absorption, for statistical purposes. In particular, one would have to eliminate the strong Ly $\alpha$ systems because of selection bias and either (1) establish a minimum detectable equivalent width which pertains to specified wavelength ranges in the spectra which are better than some minimum quality (e.g., as in SYBT or LTW) or (2) conduct an analysis which can be interpreted on the basis of simulated spectra (e.g., Paper II performs such an analysis on the sample of spectra presented in Paper I).

In each object, Ly $\alpha$ emission is the dominant feature, separating the region of high absorption-line density shortward of $\operatorname{Ly} \alpha$ emission (the Ly $\alpha$ forest) from the region longward of Ly $\alpha$ in which absorption lines are relatively rare. The existence of $\mathrm{Ly} \beta / \mathrm{O}$ VI emission in the $\mathrm{Ly} \alpha$ forest region often complicates the derivation of absorption-line lists. Emission lines due to $\mathrm{N}$ v, $\mathrm{O}$ I, $\mathrm{Si}$ IV/O IV], and $\mathrm{C}$ IV are often present in the region longward of $\mathrm{Ly} \alpha$ emission. The algorithm used to construct the absorption-line lists is explicitly detailed in Paper IV; in brief, it works as follows. A spline is fitted to each spectrum and interpolated across the regions where candidate damped Ly $\alpha$ absorption lines appear. Normally the spline provides an adequate fit to the emission lines; in a few cases where emission lines were narrow, a Gaussian was used to fit the lines. An iterative technique is used to make successive fits, excluding regions of the spectrum where deep absorption lines occur. Voigt damping profiles are then fitted to the candidate damped Ly $\alpha$ absorption lines. Details of the algorithm used to obtain the Voigt profile fits are also discussed in Paper IV. The initial wavelength centroids of the fits to candidate damped Ly $\alpha$ absorption lines are set by the location of the wings in the observed profile, but the final wavelength centroids account for the redshift determined from associated metal lines. The continua, which include the fits to candidate damped Ly $\alpha$ absorption lines, are the ones used for the purpose of deriving the absorption-line lists. The equivalent widths of the absorption lines are measured directly, integrating over the region below the continuum. Blends of lines are broken up into multiple components only when a clear reversal in the absorption-line profile occurs (obviously this is subjective and depends on the signal-to-noise ratio of the data), when the individual com-
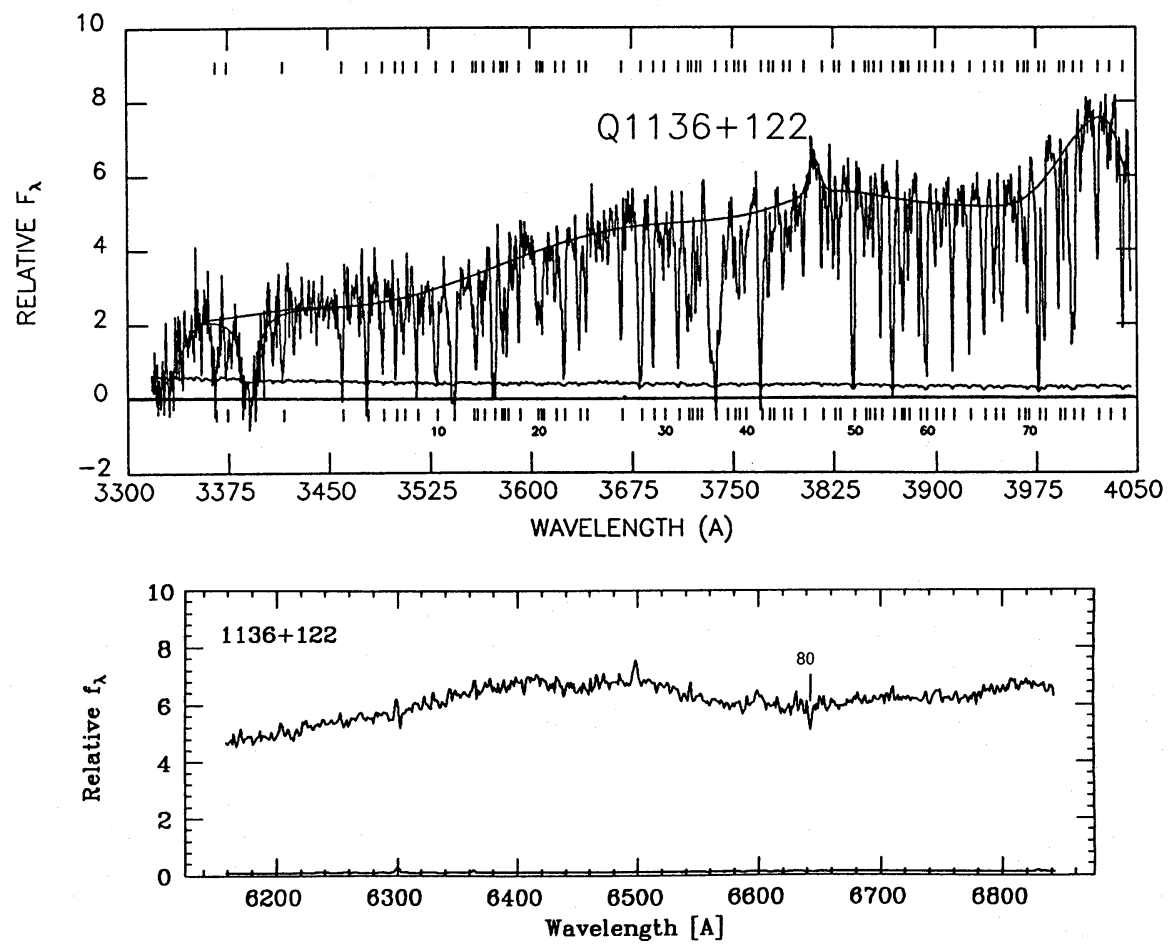

Fig. 2.-Spectrum of Q1136+113 obtained at $1.0 \AA$ resolution with the MMT $(\lambda<4050 \AA)$ and at $2.4 \AA$ resolution with the CTIO $4 \mathrm{~m}$ telescope $(\lambda>6150 \AA)$. The spectrum is unsmoothed, plotted with 2 pixels per resolution element. The $1 \sigma$ error array per pixel in the flux, the adopted continuum, and the fit to the damped Ly $\alpha$ profile are also shown. 

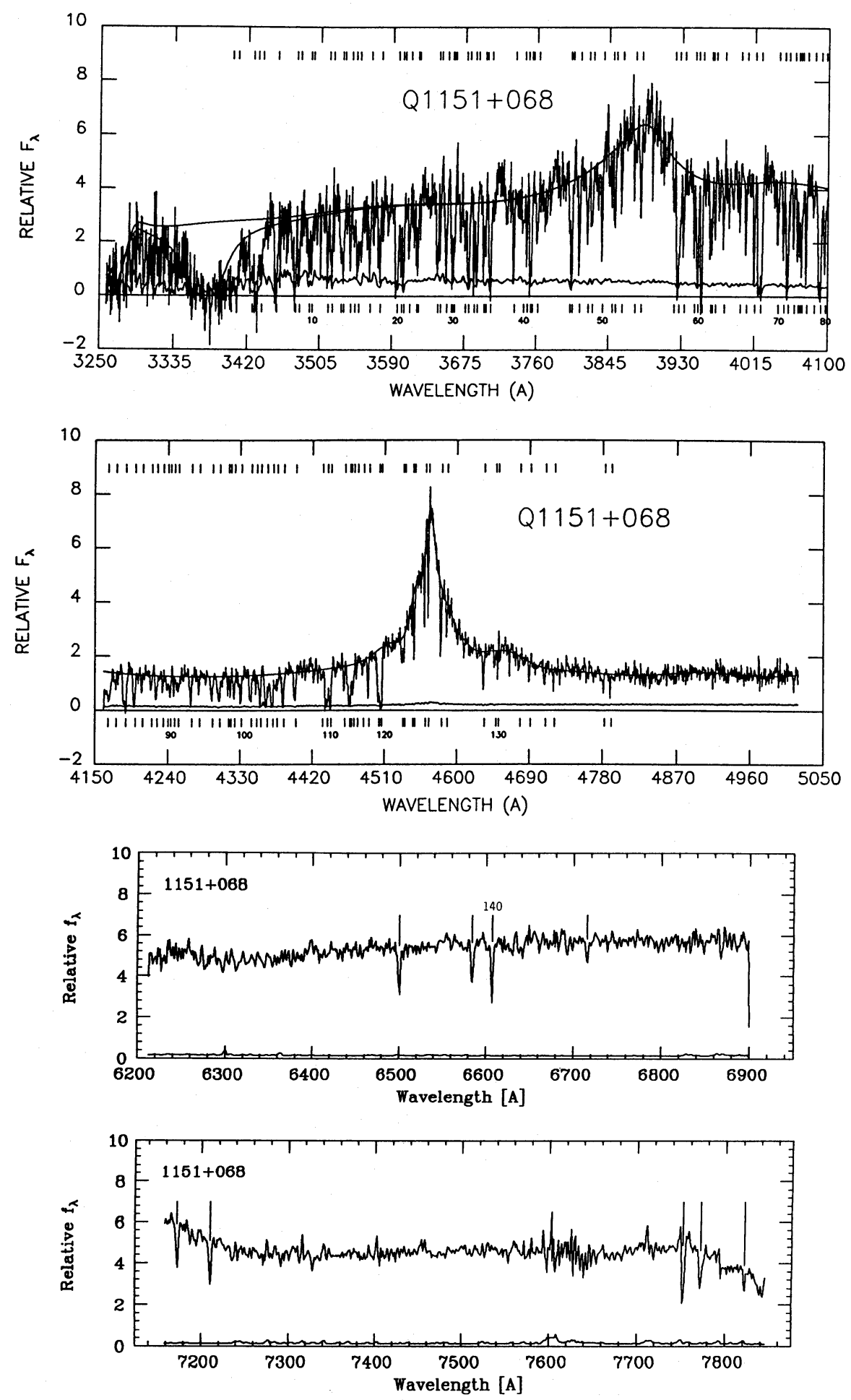

Fig. 3. - Spectrum of Q1151+068 obtained at $1.0 \AA$ resolution with the MMT $(\lambda<5125 \AA)$ and at $2.4 \AA$ resolution with the CTIO $4 \mathrm{~m}$ telescope $(\lambda>6200 \AA)$. The spectrum is unsmoothed, plotted with 2 pixels per resolution element. The $1 \sigma$ error array per pixel in the flux, the adopted continuum, and the fit to the damped Ly $\alpha$ profile are also shown.

ponents are separated by more than a resolution element, and when each of the multiple lines is statistically significant. Lines are rejected if they have a measured FWHM less than one-half of the resolution element. The wavelengths of the absorption lines are set equal to the centroids of Gaussians that are fitted to the absorption features. The errors in the equivalent widths are computed from the observed flux and associated errors with formulae derived from Poisson counting statistics (cf. Young et al. 1979); the errors in the wavelengths are derived from the least-squares fits, including both the error resulting from the initial wavelength calibration and the error derived from the Gaussian fitted to an absorption line. Tables 2-7 

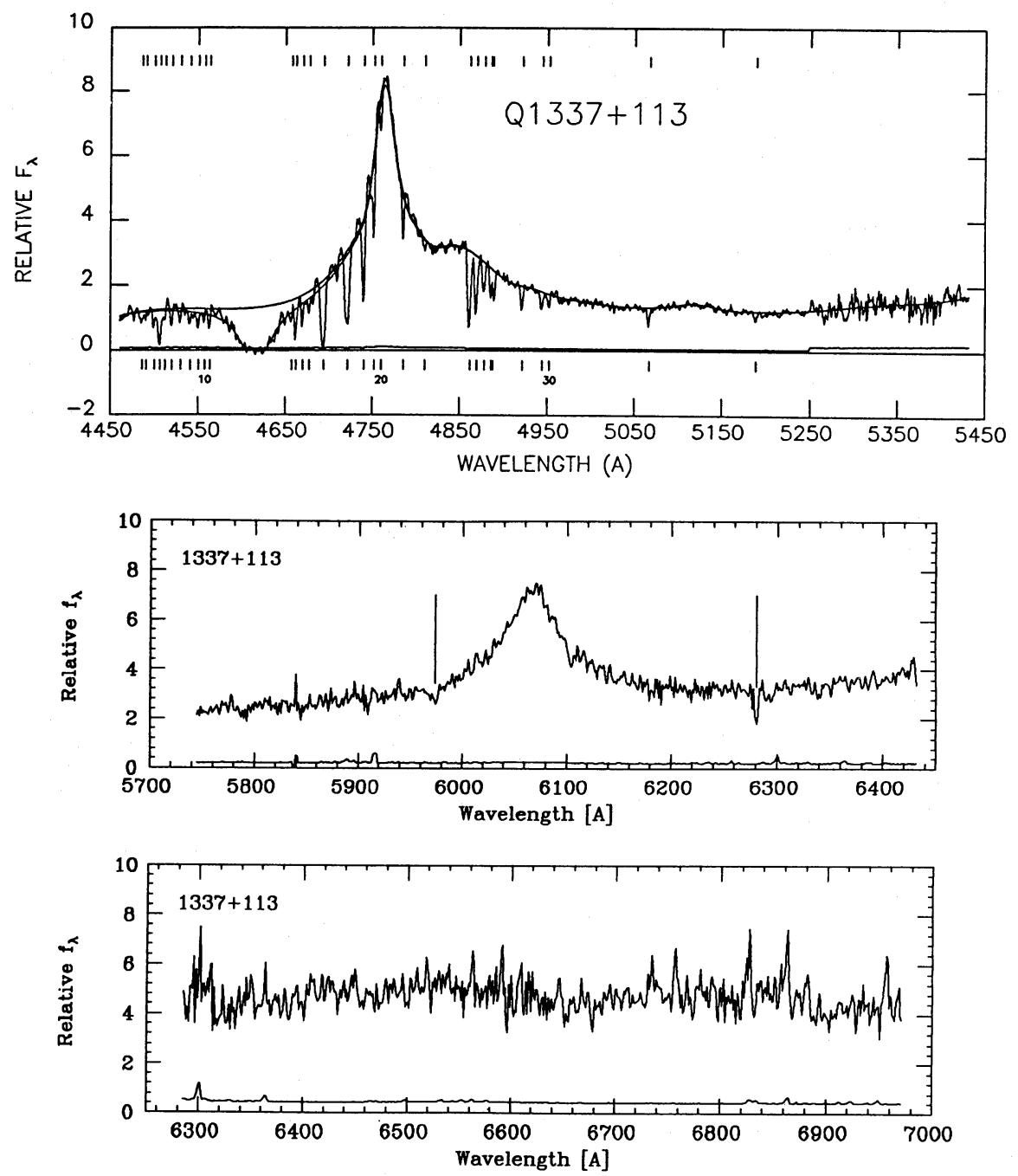

FIG. 4.-Spectrum of Q1337+113 obtained at $2.0 \AA$ resolution with the MMT $(\lambda<5450 \AA)$ and at $2.4 \AA$ resolution with the CTIO $4 \mathrm{~m}$ telescope $(\lambda>5750 \AA)$. The spectrum is unsmoothed, plotted with 2 pixels per resolution element. The $1 \sigma$ error array per pixel in the flux, the adopted continuum, and the fit to the damped Ly $\alpha$ profiles are also shown.

present the absorption-line lists for the six QSOs, listing all of these quantities along with the signal-to-noise ratio of the continuum which includes the fit to any candidate damped Ly $\alpha$ absorptions. The FWHM measurements are specified to the nearest quarter-pixel. Only lines which have a significance $\geq 4 \sigma$ in the MMT data and $\geq 5 \sigma$ in the CTIO data are labeled with a number in the line lists. These same lines are labeled on Figures 1-6. In some objects features with equivalent widths having a significance less than $4 \sigma$ (or $5 \sigma$ for the CTIO data) are included in the absorption-line lists (and denoted with a double asterisk instead of a number) if they appear to be present at wavelengths predicted on the basis of the candidate damped Ly $\alpha$ redshift. Some absorption features in the red-wavelength CTIO data with a formal significance $\geq 5 \sigma$ were disregarded because sky subtraction was deemed to give poorer accuracy than the noise array indicated (e.g., see the constraints imposed by LTW). All wavelengths listed have been reduced to heliocentric vacuum values.

In general, absorption features shortward of Ly $\alpha$ emission are "unidentified," except in cases where they coincide in wavelength with lines associated with identified metal line systems. Although the lines longward of Ly $\alpha$ emission are often metal lines associated with the damped systems, a number of cases exist where metal lines at unrelated redshifts are present. These redshift systems are noted in the tables but are not analyzed here. The "unidentified" absorption lines are mainly the Ly $\alpha$ forest lines, i.e., Ly $\alpha$ lines arising in gas apparently devoid of heavy elements (cf. SYBT). In the future we will analyze the statistical properties of the Ly $\alpha$ forest based on the new observations presented here and in Paper IV. The analysis will account for the biases caused by variable signal-to-noise ratio data and the presence of strong Ly $\alpha$ absorption. In the remaining sections of this paper, absorption-line systems which have tentatively been identified as containing damped Ly $\alpha$ absorption are dealt with exclusively.

\section{THE NATURE OF THE STRONG LYMAN-ALPHA ABSORPTION-LINE SYSTEMS}

a) Criteria for Damped Lyman-Alpha Absorption

Intermediate-resolution optical spectroscopy provides at least three tests of the damping hypothesis, as discussed below.

\section{i) Quality of the Fit}

For the damping hypothesis to be correct, the fit of a Voigt damping profile to the intermediate-resolution data must be 

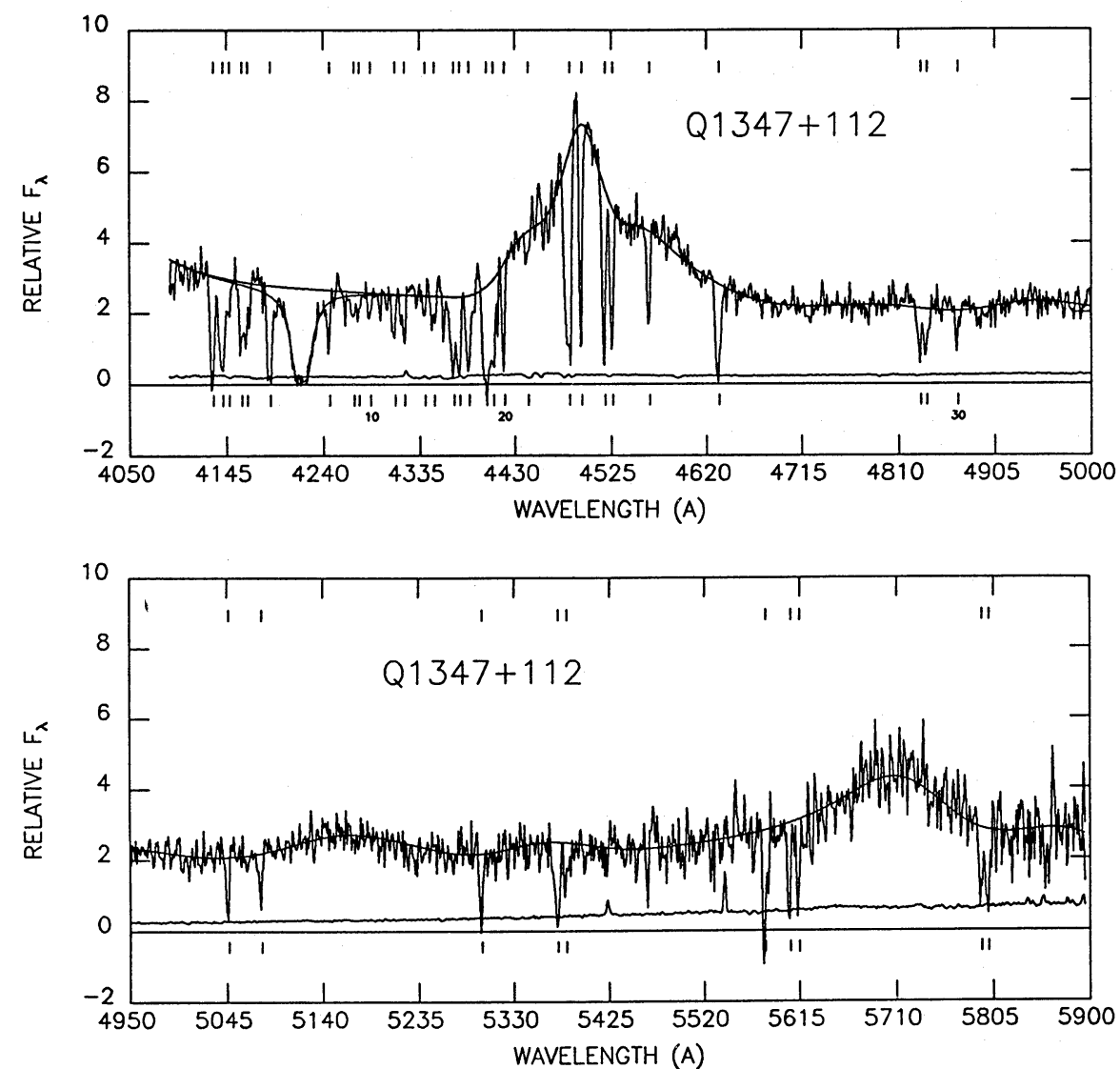

FIG. 5.-Spectrum of Q1347+112 obtained at $2.0 \AA$ resolution with the MMT. The spectrum is unsmoothed, plotted with 2 pixels per resolution element. The $1 \sigma$ error array per pixel in the flux, the adopted continuum, and the fit to the damped Ly $\alpha$ profile are also shown.

satisfactory. That is, except for the presence of weak Ly $\alpha$ forest absorption features and expected fluctuations due to finite signal-to-noise ratio data, the strong absorption feature must exhibit the divergent wings and black core characteristic of absorption lines broadened by radiation damping.

ii) Comparison of Metal Line and Inferred $\mathrm{H}$ I Velocity Profiles

If metal lines are present in a strong Ly $\alpha$ absorption system, comparison between their velocity profiles and the inferred velocity profile of $\mathrm{Ly} \alpha$ should allow a $\mathrm{Ly} \alpha$ line that is damped to be distinguished from one that arises in a velocitybroadened multicomponent system. In particular, the redshift of the metal lines should be in good agreement with the redshift of $\mathrm{Ly} \alpha$ if the $\mathrm{Ly} \alpha$ line is damped.

The expectation of comparing metal line and Ly $\alpha$ line redshifts is as follows. Multicomponent systems create broad Ly $\alpha$ lines through $\mathrm{H}$ I absorption by a series of narrow-line clouds that span the velocity interval $\Delta v_{\mathrm{HI}}>1200 \mathrm{~km} \mathrm{~s}^{-1}$. A metal line is produced by a subset of clouds that are optically thick in a specific resonance transition. These clouds span the velocity interval $\Delta v_{\text {metals }}$, where $\Delta v_{\text {metals }} \leq \Delta v_{\mathrm{H} \text { I }}$, and where the velocity intervals are defined as the FWHM of the appropriate profile. High-resolution spectroscopy of multicomponent systems shows that $v_{\text {diff }}$, the typical displacement between the velocity centroids measured from the Ly $\alpha$ and metal line profiles, is comparable to $\Delta v_{\mathrm{HI}}$ (Bechtold, Green, and York 1987; Bechtold 1988).

Now suppose that $N(\mathrm{H}$ I) in one of the clouds optically think in a metal line transition is so large that Ly $\alpha$ is optically thick in the damping wings. In that case Ly $\alpha$ absorption from the entire system will be dominated by a damping profile which is symmetric with respect to the velocity of the opaque cloud. For $N(\mathrm{H} \mathrm{I}) \geq 2 \times 10^{20} \mathrm{~cm}^{-2}, \Delta v_{\mathrm{H}}$ is set by the damping wings and will exceed $\sim 1750 \mathrm{~km} \mathrm{~s}^{-1}$, which is large compared with the $\sim 100-500 \mathrm{~km} \mathrm{~s}^{-1}$ that typifies $\Delta v_{\text {metals }}$ (cf. Young, Sargent, and Boksenberg 1982; Sargent, Boksenberg, and Steidel 1988). As a result, in a damped system the velocity centroids of the metal lines and $\operatorname{Ly} \alpha$ should coincide to within an accuracy given by $\Delta v_{\text {metals }}$. More specifically, if $z_{\text {metals }}$ is the redshift determined from the metal lines and $z_{\alpha}$ is an independent redshift determined by the Voigt damping profile fitted to Ly $\alpha$, the velocity difference between the two redshifts should be

$$
v_{\text {diff }} \simeq\left(z_{\text {metals }}-z_{\alpha}\right)\left(1+z_{\text {metals }}\right)^{-1} c<\Delta v_{\text {metals }} \ll \Delta v_{\mathrm{HI}} .
$$

Equation (1) ignores the measurement error in $z_{\alpha}$, which could be large given the confusion of the Ly $\alpha$ forest.

iii) Consistency with Higher Order Lyman Lines

If the $\mathrm{H}$ I column density is large enough, not only will damped $\operatorname{Ly} \alpha$ result, but $\operatorname{Ly} \beta$ and the other higher order Lyman lines will exhibit an appropriate Voigt profile. Therefore, if the Ly $\alpha$ line is damped, the pattern of equivalent widths and profiles for the observed Lyman lines should be consistent with (1) radiation damping for the stronger members of the Lyman series and (2) saturated absorption by a gas with an appropriate velocity dispersion for the weaker Lyman lines.

In principle these three criteria are rigorous tests of the damping hypothesis. They are not, however, always easy to 
TABLE 2

ABSORPTION-LINE LIST FOR Q0836+113

\begin{tabular}{|c|c|c|c|c|c|c|c|c|}
\hline Line No. & $\lambda(\AA)$ & $\sigma_{\lambda}(\AA)$ & $\mathrm{E}(\AA)$ & $\sigma_{E}(\AA)$ & FWHM $(\AA)$ & $\mathrm{STN}^{a}$ & Identification & $\mathbf{z}_{a b s}$ \\
\hline 1 & 3714.2 & 0.5 & 2.40 & 0.22 & 1.5 & 9.1 & ............. & ...... \\
\hline 2 & 3724.7 & 0.6 & 3.69 & 0.26 & 10.0 & 9.3 & ............ & ...... \\
\hline 3 & 3758.9 & 0.6 & 1.52 & 0.21 & 7.5 & 9.6 & ............. & $\ldots \ldots$ \\
\hline 4 & 3773.4 & 0.5 & 1.32 & 0.17 & 3.0 & 10.7 & ........... & ...... \\
\hline 5 & 3786.0 & 0.5 & 1.35 & 0.12 & 2.0 & 11.0 & ............ & ...... \\
\hline 6 & 3796.2 & 0.5 & 1.21 & 0.11 & 3.5 & 11.8 & .............. & ....... \\
\hline 7 & 3824.6 & 0.5 & 5.73 & 0.23 & 5.0 & 11.7 & MgII 2796.4 & 0.3677 \\
\hline 8 & 3835.9 & 0.5 & 3.80 & 0.21 & 4.5 & 10.5 & MgII 2803.5 & 0.3683 \\
\hline 9 & 3859.6 & 0.8 & 0.96 & 0.22 & 8.0 & 9.2 & $\ldots \ldots \ldots$ & ...... \\
\hline 10 & 3922.4 & 0.5 & 2.02 & 0.19 & 5.0 & 8.8 & ............ & ...... \\
\hline 11 & 3928.7 & 0.5 & 1.48 & 0.15 & 3.5 & 8.8 & $\ldots \ldots \ldots \ldots$ & $\ldots \ldots$ \\
\hline 12 & 3932.2 & 0.5 & 1.22 & 0.18 & 3.0 & 9.3 & ............. & ...... \\
\hline 13 & 3969.5 & 0.5 & 1.13 & 0.17 & 2.5 & 9.6 & FeII 1144.9 & 2.4671 \\
\hline 14 & 3979.3 & 0.5 & 1.04 & 0.17 & 2.5 & 8.7 & .............. & $\ldots \ldots$ \\
\hline 15 & 3994.8 & 0.5 & 2.18 & 0.24 & 6.0 & 9.0 & ............ & ...... \\
\hline 16 & 4002.0 & 0.5 & 1.68 & 0.21 & 2.5 & 8.8 & ............. & ...... \\
\hline 17 & 4019.6 & 0.6 & 3.20 & 0.24 & 6.0 & 8.8 & ............ & $\ldots . .$. \\
\hline 18 & 4024.8 & 0.5 & 2.11 & 0.19 & 4.0 & 8.6 & ............ & $\ldots . .$. \\
\hline 19 & 4037.4 & 0.6 & 1.63 & 0.22 & 6.5 & 8.7 & $\ldots \ldots \ldots$ & ....... \\
\hline 20 & 4041.3 & 0.5 & 1.39 & 0.15 & 3.0 & 7.8 & FeII 2260.8 & 0.7876 \\
\hline 21 & 4046.8 & 0.5 & 1.95 & 0.26 & 2.5 & 7.1 & ............. & $\ldots \ldots$ \\
\hline 22 & 4066.6 & 0.5 & 2.30 & 0.16 & 2.5 & 8.5 & ............ & $\ldots . .$. \\
\hline 23 & 4070.9 & 0.5 & 4.58 & 0.19 & 5.5 & 8.9 & ............ & $\ldots \ldots$ \\
\hline 24 & 4105.5 & 0.5 & 1.65 & 0.17 & 2.5 & 8.5 & ............ & ...... \\
\hline 25 & 4127.2 & 0.5 & 2.22 & 0.18 & 3.0 & 8.3 & SilI 1190.4 & 2.4671 \\
\hline 26 & 4136.5 & 0.5 & 6.31 & 0.28 & 6.5 & 8.7 & SiII 1193.3 & 2.4664 \\
\hline 27 & 4158.0 & 0.5 & 1.62 & 0.23 & 5.0 & 8.0 & ............ & ...... \\
\hline 28 & 4163.0 & 0.6 & 0.80 & 0.14 & 3.0 & 7.2 & ............ & ....... \\
\hline 29 & 4183.0 & 0.5 & 3.06 & 0.26 & 6.5 & 6.7 & SiIII 1206.5 & 2.4671 \\
\hline 30 & 4189.9 & 0.5 & 3.16 & 0.25 & 3.5 & 6.5 & FeII 2344.2 & 0.7873 \\
\hline 31 & 4232.8 & 0.5 & 1.18 & 0.25 & 2.0 & 4.1 & FeII 2367.6 & 0.7878 \\
\hline 32 & 4243.8 & 0.5 & 1.87 & 0.26 & 1.5 & 6.9 & FeII 2375.5 & 0.7872 \\
\hline 33 & 4259.2 & 0.5 & 3.87 & 0.24 & 2.5 & 8.1 & FeII 2382.8 & 0.7875 \\
\hline 34 & 4269.3 & 0.7 & 1.74 & 0.23 & 4.0 & 8.0 & ............. & ....... \\
\hline 35 & 4276.9 & 0.5 & 1.15 & 0.18 & 4.5 & 7.9 & ............ & $\ldots \ldots$ \\
\hline 36 & 4283.1 & 0.5 & 1.66 & 0.18 & 2.5 & 8.0 & ............. & ...... \\
\hline 37 & 4287.9 & 0.5 & 1.04 & 0.16 & 3.0 & 7.7 & ............ & $\ldots \ldots$ \\
\hline 38 & 4302.2 & 0.5 & 0.63 & 0.15 & 2.0 & 7.8 & ............ & $\ldots \ldots$ \\
\hline 39 & 4306.5 & 0.5 & 0.55 & 0.13 & 2.5 & 7.7 & ............ & $\ldots \ldots$ \\
\hline 40 & 4310.5 & 0.5 & 0.94 & 0.13 & 2.0 & 8.0 & ............ & $\ldots \ldots$ \\
\hline 41 & 4319.8 & 0.5 & 0.91 & 0.12 & 2.0 & 7.4 & ............ & $\ldots \ldots$ \\
\hline 42 & 4343.9 & 0.5 & 1.04 & 0.21 & 4.5 & 8.7 & ................ & $\ldots \ldots$ \\
\hline \multirow[t]{2}{*}{43} & 4369.1 & 0.5 & 2.90 & 0.22 & 2.5 & 8.7 & SiII 1260.4 & 2.4664 \\
\hline & & & & & & & CIV 1548.2: & 1.8221 \\
\hline 44 & 4376.7 & 0.5 & 1.77 & 0.17 & 2.5 & 9.2 & CIV 1550.8: & 1.8222 \\
\hline 45 & 4397.6 & 0.5 & 1.39 & 0.15 & 3.0 & 10.1 & ................ & $\ldots .$. \\
\hline 46 & 4410.9 & 0.5 & 4.80 & 0.18 & 7.0 & 12.3 & ............ & $\ldots \ldots$ \\
\hline 47 & 4429.4 & 0.5 & 0.44 & 0.09 & 2.0 & 12.0 & ............ & $\ldots \ldots$ \\
\hline 48 & 4439.0 & 0.5 & 0.81 & 0.09 & 2.5 & 14.4 & ............ & $\ldots \ldots$ \\
\hline 49 & 4442.3 & 0.5 & 0.44 & 0.07 & 2.5 & 13.6 & ............ & $\ldots \ldots$ \\
\hline 50 & 4451.9 & 0.5 & 2.40 & 0.09 & 3.0 & 19.0 & ............ & $\ldots \ldots$ \\
\hline 51 & 4514.0 & 0.5 & 2.01 & 0.12 & 2.5 & 13.0 & OI 1302.2 & 2.4664 \\
\hline 52 & 4521.7 & 0.5 & 1.26 & 0.12 & 2.0 & 12.1 & SilI 1304.4 & 2.4665 \\
\hline \multirow{2}{*}{53} & 4624.8 & 0.5 & 4.13 & 0.24 & 5.0 & 7.7 & CII 1334.5 & 2.4656 \\
\hline & & & & & & & FeII 2586.7 & 0.7879 \\
\hline 54 & 4647.5 & 0.5 & 1.86 & 0.20 & 3.0 & 6.8 & FeII 2600.2 & 0.7874 \\
\hline 55 & 4704.0 & 0.8 & 2.21 & 0.35 & 6.5 & 7.0 & $\ldots \ldots \ldots$ & $\ldots \ldots$ \\
\hline 56 & 4741.8 & 0.6 & 1.82 & 0.25 & 6.0 & 6.7 & $\ldots \ldots \ldots$ & ...... \\
\hline 57 & 4998.1 & 0.5 & 4.14 & 0.26 & 4.5 & 5.9 & MgII 2796.4 & 0.7873 \\
\hline 58 & 5011.2 & 0.5 & 3.87 & 0.34 & 4.0 & 5.9 & MgII 2803.5. & 0.7875 \\
\hline$* *$ & 5097.8 & 0.5 & 0.85 & 0.20 & 4.0 & 5.9 & Mgl 2852.1 & 0.7874 \\
\hline$* *$ & 5292.2 & 0.6 & 0.45 & 0.30 & 4.0 & 4.0 & SiII 1526.7 & 2.4664 \\
\hline 59 & 5577.3 & 0.6 & 2.82 & 0.53 & 4.5 & 3.2 & FeII 1608.5 & 2.4674 \\
\hline 60 & 8123.6 & 0.2 & 2.60 & 0.31 & 4.2 & 12.2 & FeII 2344.2 & 2.4654 \\
\hline 61 & 8227.5 & 0.2 & 2.06 & 0.36 & 3.0 & 9.1 & FeII 2374.5 & 2.4650 \\
\hline 62 & 8256.9 & 0.2 & 3.34 & 0.32 & 3.6 & 11.3 & FeII 2382.8 & 2.4653 \\
\hline
\end{tabular}

(Notes to table are on following page.) 
implement because of the difficulties involved in determining the continuum across the broad Ly $\alpha$ feature. Aside from limitations imposed by data with finite signal-to-noise ratio, the fitting of a Voigt damping profile to the observed feature is complicated by confusion noise generated by weak Ly $\alpha$ forest absorption, and/or metal line absorption in the damping wings. Furthermore, asymmetries in the Ly $\alpha$ line may be caused by neighboring emission lines (cf. Briggs, Turnshek, and Wolfe 1984) or by neighboring damped Ly $\alpha$ absorption (see the spectrum of Q2359-022 in Paper IV and the spectrum of Q2348 - 011 in this paper). Finally, Ly $\alpha$ emission originating in the absorber may fill in parts of the otherwise black core of the profile with emission (see Foltz, Chaffee, and Weymann 1986 for additional discussion).

Despite these problems, we have succeeded in fitting seven damping profiles to the data on six QSOs presented in Figures 1-6. The strength of the evidence for damped Ly $\alpha$ absorption in terms of the criteria set forth above, along with other notable characteristics of the spectra and the properties of the damped Ly $\alpha$ absorption systems, are considered in the discussion of individual systems in the next section.

\section{b) Analysis and Discussion of the Seven Damped Lyman-Alpha Systems}

For each absorption system for which a sufficient number of equivalent width measurements were available, a curve-ofgrowth analysis was performed assuming that the absorption arises in a single component of Gaussian velocity dispersion. Only lines from low-ion species (i.e., lines of $\mathrm{C}$ II, O I, Al II, Si II, $\mathrm{Mg}$ II, and Fe II) were included in the curve-of-growth analysis, because lines from high-ion species (e.g., $\mathrm{Si}$ IV and $\mathrm{C}$ IV) may originate in absorbing regions distinct from those that give rise to the low-ion lines. For each system, a least-squares fit was made to the data, taking the Doppler parameter and the column densities of the observed ions as free parameters. Note that the procedure was different from the one adopted by Black, Chafee, and Foltz (1987). Those authors chose to make a determination of the column density of an ion from each
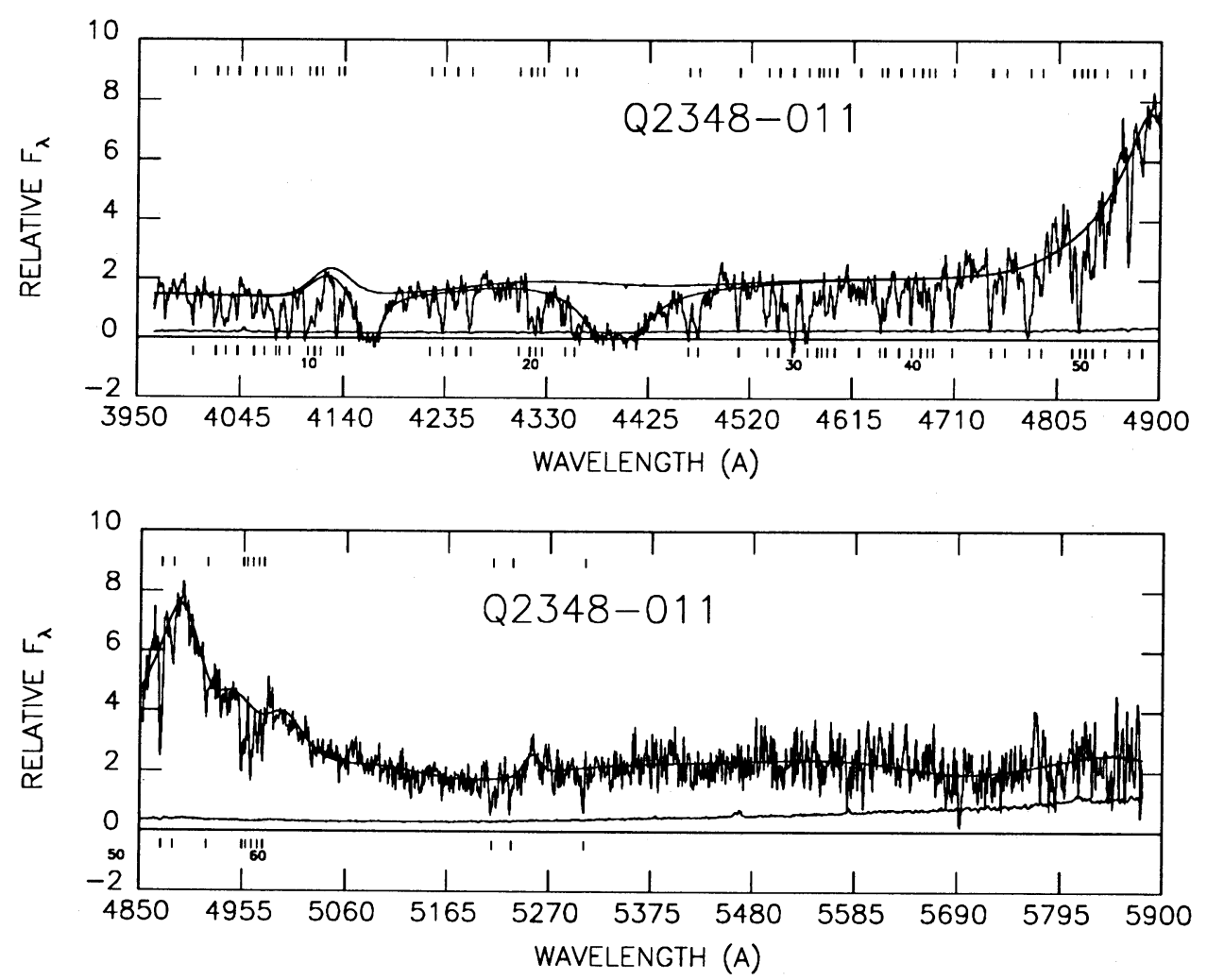

Fig. 6.-Spectrum of Q2348-011 obtained at $2.0 \AA$ resolution with the MMT. The spectrum is unsmoothed, plotted with 2 pixels per resolution element. The $1 \sigma$ error array per pixel in the flux, the adopted continuum, and the fit to the damped Ly $\alpha$ profiles are also shown.

TABLE 2.-Continued

NOTES ON INDIVIDUAL LINES

Lines 7 and 8.-This identification is based only on $\mathrm{Mg}$ II $\lambda 22796,2803$ in the Ly $\alpha$ forest; other predicted prominent lines at this redshift are not in the observed range.

Line 20.-This identification may be incorrect, since Fe II $\lambda 2260$ has a low oscillator strength.

Line 30.-This identification may be incorrect, since Fe II $\lambda 2367$ has a low oscillator strength.

Lines 43 and 44.-The C IV identification is based only on C IV $\lambda \lambda 1548,1550$ in the Ly $\alpha$ forest; no indication of Si IV $\lambda \lambda 1393,1402$ exists at this same redshift.

a Signal-to-noise ratio of the continuum spectrum, which includes the fit to candidate damped Ly $\alpha$, evaluated at $\lambda$. 
TABLE 3

ABSORPTION-LINE LiST FOR Q1136+112

\begin{tabular}{|c|c|c|c|c|c|c|c|c|}
\hline Line No. & $\lambda(\AA)$ & $\sigma_{\lambda}(\AA)$ & $\mathrm{E}(\AA)$ & $\sigma_{E}(\AA)$ & FWHM $(\AA)$ & $\mathrm{STN}^{a}$ & Identification & $\mathrm{z}_{a b s}$ \\
\hline 1 & 3365.7 & 0.3 & 2.79 & 0.37 & 4.0 & 2.7 & ............ & ....... \\
\hline 2 & 3374.2 & 0.3 & 1.34 & 0.30 & 1.8 & 2.3 & ............. & ....... \\
\hline 3 & 3416.1 & 0.3 & 2.13 & 0.30 & 3.5 & 3.3 & ............ & ....... \\
\hline 4 & 3460.4 & 0.3 & 2.58 & 0.27 & 4.0 & 3.9 & ............ & ....... \\
\hline 5 & 3478.7 & 0.2 & 2.36 & 0.18 & 2.3 & 4.2 & ............ & $\ldots \ldots$ \\
\hline 6 & 3490.6 & 0.2 & 1.18 & 0.17 & 1.5 & 4.0 & ............. & $\ldots \ldots$ \\
\hline 7 & 3500.2 & 0.2 & 1.33 & 0.19 & 1.5 & 4.3 & ............ & $\ldots \ldots$ \\
\hline 8 & 3506.2 & 0.3 & 1.19 & 0.24 & 4.0 & 4.3 & ............ & ...... \\
\hline 9 & 3515.8 & 0.2 & 1.69 & 0.19 & 1.8 & 4.7 & ............. & ....... \\
\hline 10 & 3530.4 & 0.2 & 2.70 & 0.17 & 3.5 & 5.3 & ............. & ........ \\
\hline 11 & 3542.9 & 0.3 & 5.58 & 0.23 & 5.3 & 5.7 & ............ & $\ldots \ldots$ \\
\hline 12 & 3557.4 & 0.2 & 0.49 & 0.11 & 1.3 & 5.1 & ............ & ....... \\
\hline 13 & 3559.8 & 0.2 & 1.85 & 0.15 & 2.5 & 5.7 & ............ & ....... \\
\hline 14 & 3565.3 & 0.2 & 0.43 & 0.10 & 0.8 & 5.0 & ............. & ...... \\
\hline 15 & 3573.2 & 0.3 & 3.77 & 0.15 & 4.0 & 6.9 & ............. & ....... \\
\hline 16 & 3578.4 & 0.2 & 0.52 & 0.09 & 1.3 & 5.4 & ............ & ....... \\
\hline 17 & 3580.1 & 0.2 & 1.03 & 0.11 & 1.5 & 6.1 & ................. & ....... \\
\hline 18 & 3583.0 & 0.2 & 1.31 & 0.12 & 1.8 & 6.6 & ............. & ....... \\
\hline 19 & 3591.8 & 0.2 & 0.91 & 0.10 & 1.5 & 6.7 & ............. & ....... \\
\hline 20 & 3605.3 & 0.2 & 0.83 & 0.10 & 1.8 & 6.9 & ............ & ....... \\
\hline 21 & 3607.6 & 0.3 & 0.99 & 0.10 & 1.8 & 6.8 & ............ & ....... \\
\hline 22 & 3609.4 & 0.2 & 0.72 & 0.10 & 1.3 & 7.0 & ............ & ....... \\
\hline 23 & 3618.7 & 0.2 & 0.68 & 0.10 & 1.8 & 6.9 & .............. & ....... \\
\hline 24 & 3625.2 & 0.2 & 2.86 & 0.16 & 3.5 & 7.8 & ............. & ....... \\
\hline 25 & 3636.6 & 0.2 & 1.73 & 0.11 & 3.0 & 7.7 & ............. & ....... \\
\hline 26 & 3641.9 & 0.2 & 0.93 & 0.11 & 1.5 & 7.4 & ................. & ....... \\
\hline 27 & 3667.9 & 0.2 & 1.38 & 0.11 & 2.8 & 7.9 & SiII 1193.3 & 2.0737 \\
\hline 28 & 3682.3 & 0.2 & 3.50 & 0.13 & 4.0 & 9.4 & MgII 2796.4 & 0.3168 \\
\hline 29 & 3691.6 & 0.2 & 2.02 & 0.12 & 2.5 & 9.0 & MgII 2803.5 & 0.3168 \\
\hline 30 & 3699.6 & 0.3 & 0.66 & 0.12 & 4.5 & 8.4 & $\ldots \ldots \ldots$ & ....... \\
\hline 31 & 3710.1 & 0.2 & 2.18 & 0.12 & 2.5 & 8.8 & ............ & $\ldots \ldots$ \\
\hline 32 & 3717.3 & 0.2 & 1.42 & 0.11 & 1.3 & 8.8 & ............. & ....... \\
\hline 33 & 3719.8 & 0.2 & 1.36 & 0.09 & 2.3 & 9.0 & ............ & ....... \\
\hline 34 & 3723.6 & 0.2 & 0.77 & 0.07 & 1.8 & 8.6 & ............. & ....... \\
\hline 35 & 3726.5 & 0.2 & 0.83 & 0.10 & 2.5 & 8.9 & .............. & ....... \\
\hline 36 & 3737.5 & 0.3 & 8.23 & 0.17 & 8.8 & 10.3 & HI 1215.7 & 2.0744 \\
\hline 37 & 3746.0 & 0.2 & 0.69 & 0.09 & 2.8 & 8.7 & ............. & ...... \\
\hline 38 & 3751.7 & 0.2 & 0.39 & 0.07 & 1.0 & 8.1 & ............ & ....... \\
\hline 39 & 3754.9 & 0.2 & 1.40 & 0.11 & 4.0 & 9.5 & ............. & $\ldots \ldots$ \\
\hline 40 & 3759.8 & 0.3 & 1.15 & 0.12 & 3.5 & 9.3 & .............. & ....... \\
\hline 41 & 3771.7 & 0.2 & 3.43 & 0.11 & 2.5 & 10.7 & .............. & ....... \\
\hline 42 & 3777.5 & 0.2 & 0.94 & 0.08 & 3.3 & 9.8 & ............ & ....... \\
\hline 43 & 3780.8 & 0.3 & 0.61 & 0.10 & 3.3 & 9.4 & ............ & ....... \\
\hline 44 & 3788.4 & 0.2 & 0.99 & 0.10 & 2.0 & 9.7 & .............. & $\ldots \ldots$. \\
\hline 45 & 3793.0 & 0.3 & 0.78 & 0.10 & 3.5 & 9.8 & .............. & $\ldots \ldots$ \\
\hline 46 & 3803.7 & 0.2 & 0.77 & 0.07 & 1.8 & 10.2 & ............ & $\ldots \ldots$ \\
\hline 47 & 3817.2 & 0.2 & 0.54 & 0.06 & 1.3 & 11.4 & ............. & ....... \\
\hline 48 & 3825.9 & 0.2 & 0.63 & 0.07 & 1.3 & 10.4 & ............ & ....... \\
\hline 49 & 3829.7 & 0.2 & 0.74 & 0.09 & 1.5 & 10.7 & .............. & $\ldots \ldots$ \\
\hline 50 & 3840.3 & 0.2 & 2.83 & 0.07 & 3.0 & 13.8 & ............ & $\ldots \ldots$ \\
\hline 51 & 3848.5 & 0.2 & 0.41 & 0.08 & 1.0 & 10.3 & ............ & $\ldots \ldots$ \\
\hline 52 & 3851.3 & 0.2 & 0.37 & 0.06 & 2.0 & 10.1 & ............. & $\ldots \ldots$ \\
\hline 53 & 3855.0 & 0.2 & 0.35 & 0.05 & 1.0 & 10.0 & ............. & ....... \\
\hline 54 & 3860.2 & 0.2 & 1.06 & 0.07 & 1.3 & 11.1 & ............. & $\ldots \ldots$. \\
\hline 55 & 3868.9 & 0.2 & 2.69 & 0.08 & 2.8 & 12.7 & ................. & ....... \\
\hline 56 & 3874.8 & 0.2 & 0.71 & 0.06 & 0.8 & 11.1 & SiII 1260.4 & 2.0734 \\
\hline 57 & 3876.8 & 0.2 & 1.16 & 0.06 & 1.8 & 11.8 & .................. & ...... \\
\hline 58 & 3880.6 & 0.2 & 0.53 & 0.06 & 1.3 & 10.5 & ............. & ....... \\
\hline 59 & 3888.4 & 0.2 & 0.89 & 0.06 & 1.5 & 11.5 & ............ & $\ldots \ldots$ \\
\hline 60 & 3893.2 & 0.2 & 3.23 & 0.09 & 4.0 & 13.3 & ............ & ....... \\
\hline 61 & 3900.3 & 0.2 & 0.54 & 0.08 & 1.3 & 10.6 & ............ & $\ldots \ldots$ \\
\hline 62 & 3905.2 & 0.2 & 0.35 & 0.07 & 1.0 & 10.4 & ................. & ........ \\
\hline 63 & 3913.2 & 0.2 & 1.70 & 0.08 & 1.8 & 11.9 & ............. & $\ldots \ldots$ \\
\hline 64 & 3925.8 & 0.2 & 1.25 & 0.06 & 1.5 & 12.0 & ............ & $\ldots \ldots$ \\
\hline 65 & 3936.6 & 0.2 & 1.86 & 0.07 & 2.5 & 13.5 & ............... & ....... \\
\hline 66 & 3944.4 & 0.3 & 1.10 & 0.08 & 2.0 & 12.0 & ............. & $\ldots \ldots$ \\
\hline 67 & 3950.0 & 0.2 & 1.61 & 0.08 & 3.0 & 12.4 & ................ & ....... \\
\hline
\end{tabular}


TABLE 3.-Continued

\begin{tabular}{|c|c|c|c|c|c|c|c|c|}
\hline Line No. & $\lambda(\AA)$ & $\sigma_{\lambda}(\AA)$ & $E(\AA)$ & $\sigma_{E}(\AA)$ & FWHM $(\AA)$ & $\mathrm{STN}^{a}$ & Identification & $\mathbf{z}_{a b s}$ \\
\hline 68 & 3961.6 & 0.2 & 0.38 & 0.05 & 1.8 & 11.0 & ............ & $\ldots \ldots$ \\
\hline 69 & 3966.6 & 0.2 & 0.56 & 0.06 & 2.0 & 12.0 & ............. & $\ldots \ldots$ \\
\hline 70 & 3969.3 & 0.2 & 1.07 & 0.06 & 1.8 & 13.6 & ............ & ...... \\
\hline 71 & 3977.4 & 0.2 & 2.71 & 0.06 & 3.0 & 16.1 & ............ & $\ldots \ldots$ \\
\hline 72 & 3981.8 & 0.2 & 1.92 & 0.06 & 2.8 & 15.0 & ............. & $\ldots \ldots$ \\
\hline 73 & 3992.5 & 0.2 & 1.05 & 0.05 & 1.8 & 14.8 & ............. & $\ldots \ldots$ \\
\hline 74 & 3996.4 & 0.2 & 0.73 & 0.06 & 1.3 & 14.7 & ............ & ....... \\
\hline 75 & 4003.2 & 0.3 & 3.39 & 0.07 & 4.5 & 18.0 & OI 1302.2 & 2.0742 \\
\hline 76 & 4009.7 & 0.2 & 0.25 & 0.04 & 1.0 & 14.5 & SiII 1304.4 & 2.0740 \\
\hline 77 & 4021.8 & 0.2 & 1.26 & 0.06 & 2.8 & 17.3 & .............. & $\ldots \ldots$ \\
\hline 78 & 4030.4 & 0.2 & 0.20 & 0.04 & 1.0 & 14.9 & ............. & $\ldots \ldots$ \\
\hline 79 & 4040.6 & 0.2 & 1.48 & 0.05 & 2.0 & 16.8 & $\ldots \ldots \ldots$ & $\ldots \ldots$ \\
\hline 80 & 6643.2 & 0.3 & 0.42 & 0.05 & 2.4 & 53.3 & FeII 2382.8 & 1.7890 \\
\hline
\end{tabular}

Notes ON INDIVIDUAL LINES

Lines 28 and 29.-This identification is based only on $\mathrm{Mg}$ il $\lambda \lambda 2796,2803$ in the $\mathrm{Ly} \alpha$ forest; other predicted prominent lines at this redshift are not in the observed range.

a Signal-to-noise ratio of the continuum spectrum, which includes the fit to candidate damped Ly $\alpha$, evaluated at $\lambda$.

transition observed, while in our analysis the column density of a particular ion with more than one observed transition is a free parameter determined in the least-squares fit.

The properties of the seven damped Ly $\alpha$ systems, identified in the six QSO spectra studied here, are presented in Tables $8-15$. Table 8 lists the mean absorption redshift, $z_{\text {abs }}$, derived from lines longward of Ly $\alpha$ emission, and the rest-frame equivalent widths, $W$, and associated statistical errors, $\sigma_{W}$, for a variety of metal line transitions that are used in the leastsquares curve-of-growth analysis. The metal line rest-frame equivalent widths in Table 8 are simply derived from the line lists, except in cases where a line is not present to a significance greater than $4 \sigma$ (or $5 \sigma$ for the CTIO data). In these instances the equivalent widths were remeasured at the predicted wavelengths over the velocity interval $\delta v$. The parameter $\delta v$ is a velocity interval which was chosen, based on inspection of the data, to include all absorption which might be present at the system redshift. The value of $\delta v$ is listed in Table 8 . The equivalent width may be negative if positive noise is sufficiently strong. The quoted errors in equivalent widths are $1 \sigma$ errors derived from the photon-counting statistics. Table 9 contains $z_{\text {abs }} ; \Delta v_{\text {metals }}$, which is the FWHM of the low-ion profiles (normally measured from $\mathrm{C}$ II, Si II, or $\mathrm{Fe} \mathrm{II}$ ); $\Delta v_{\mathrm{HI}}$, which is the observed inferred FWHM velocity extent of the Ly $\alpha$ absorption; the parameter $v_{\text {diff }}$ as defined in equation (1); and the neutral hydrogen column density, $N(\mathrm{H} \mathrm{I})$, deduced from the Voigt fit to the damping profile. Table 10 contains a summary of the effective velocity dispersion, $\sigma$, and formal upper and lower error limits deduced from the curve-of-growth analysis for each of five systems for which a curve-of-growth analysis was performed. (Note that the velocity dispersion, $\sigma$, is related to the often-used Doppler parameter $b$, such that $b=2^{1 / 2} \sigma$.) Tables 11-15 contain the derived best-fit column densities for the low ions for each of the five damped Ly $\alpha$ systems. The oscillator strengths, $f$, used in the curve-of-growth analysis and listed in Table 8, are those recently compiled by Morton, York, and Jenkins (1988). As is the usual practice when performing a curve-of-growth analysis, relative abundances were allowed to remain free parameters. Therefore, the derived $\sigma$ is based on low ions for which two or more transitions are observed to be present (e.g., for Si II, Fe II, or Mg II). Note that other low ions for which only a single transition is measured are also plotted on the curves of growth. Lanzetta, Wolfe, and Turnshek (1989) discuss the method employed to perform the curve-of-growth analysis in somewhat more detail. The ionic column densities, $\log N$, presented in Tables $11-15$ should probably strictly be viewed as lower limits to the actual values, since an undetected very low velocity dispersion component could be present (see $\S$ IV for more discussion). Tables 11-15 also list the formal errors in column density, $+\sigma_{\log N}$ and $-\sigma_{\log N}$. Given the selfconsistency of the data, these errors are not realistic. Tables 11-15 also list the minimum column density of an ion, log $N_{\min }$, derived by assuming that the weakest observed line lies on the linear part of the curve of growth, and the maximum column density of an ion, $\log N_{\max }$, derived by assuming that the strongest observed line lies on the damping part of the curve of growth. In no case could a better upper limit for log $N_{\max }$ be obtained by actually fitting Voigt profiles to data. The following is a discussion of the seven damped Ly $\alpha$ systems that were studied.

$$
\text { i) The } Q 0836+113 . z_{\text {abs }}=2.4660 \text { System }
$$

The Ly $\alpha$ feature is well fitted by a damping profile with $N\left(\mathrm{H}_{\mathrm{I}}\right) \simeq 4 \times 10^{20} \mathrm{~cm}^{-2}$. However, the presence of three or four Fe II absorption lines at $z_{\text {abs }}=0.787$ and $\mathrm{Si}$ III $\lambda 1206$ absorption at $z_{\text {abs }}=2.4660$ causes depressions in the wings of the $\mathrm{Ly} \alpha$ absorption profile and confuses the fit. Additional evidence indicating damping is that $v_{\text {diff }}<50 \mathrm{~km} \mathrm{~s}^{-1}$ and $\Delta v_{\text {metals }} \leq 150 \mathrm{~km} \mathrm{~s}^{-1}$, while $\Delta v_{\mathrm{HI}} \simeq 2500 \mathrm{~km} \mathrm{~s}^{-1}$. In Figure 7 the small value of $v_{\text {diff }}$ is illustrated by plotting the velocity profile of the $\mathrm{O}_{\text {I }} \lambda 1302$ absorption line and the inferred velocity profile of Ly $\alpha$ on top of one another. (Note that in Fig. 7 the data containing the $z=2.4660 \mathrm{Si}$ II $\lambda 1304$ line is not plotted.) The system is characterized by strong, nearly resolved low-ion absorption and weak or absent high-ion absorption. The curve-of-growth analysis, illustrated in Figure 8, is based on six $\mathrm{Fe}$ II line measurements and five $\mathrm{Si}$ II line measurements. An effective velocity dispersion $\sigma \simeq 50 \mathrm{~km} \mathrm{~s}^{-1}$ is found. However, if only the six Fe II lines are used in the curve-of-growth analysis, an effective velocity dispersion $\sigma \simeq 20 \mathrm{~km} \mathrm{~s}^{-1}$ is found. The curve-of-growth analysis applied to the $3 \sigma$ upper limit on $\mathrm{C}$ IV absorption suggests that $N\left(\mathrm{C}^{+}\right) / N\left(\mathrm{C}^{+3}\right)>16$. Note that the measured equivalent width of the $C$ II $\lambda .1334$ absorption line at $z_{\text {abs }}=2.4660$ is probably too large, since it is 
TABLE 4

ABSORPTION LINE LIST FOR Q1151 + 068

\begin{tabular}{|c|c|c|c|c|c|c|c|c|}
\hline Line No. & $\lambda(\AA)$ & $\sigma_{\lambda}(\AA)$ & $\mathrm{E}(\AA)$ & $\sigma_{E}(\AA)$ & FWHM $(\AA)$ & $\operatorname{STN}^{a}$ & Identification & $\mathrm{z}_{a b s}$ \\
\hline 1 & 3402.0 & 0.3 & 4.27 & 0.19 & 4.8 & 5.5 & ................... & $\ldots \ldots$ \\
\hline 2 & 3408.4 & 0.3 & 6.16 & 0.60 & 8.5 & 2.4 & .............. & ....... \\
\hline 3 & 3426.3 & 0.3 & 3.54 & 0.17 & 5.3 & 7.0 & HI 1215.7 & 1.8184 \\
\hline 4 & 3431.9 & 0.3 & 5.58 & 0.28 & 6.8 & 4.8 & ................. & ....... \\
\hline 5 & 3437.3 & 0.3 & 2.07 & 0.37 & 3.3 & 2.7 & .............. & ....... \\
\hline 6 & 3455.0 & 0.3 & 4.13 & 0.25 & 4.8 & 5.8 & ................. & ....... \\
\hline 7 & 3477.3 & 0.3 & 2.59 & 0.18 & 2.8 & 5.1 & .............. & ....... \\
\hline 8 & 3481.8 & 0.3 & 2.07 & 0.21 & 2.8 & 3.8 & .............. & ....... \\
\hline 9 & 3493.9 & 0.3 & 1.55 & 0.24 & 3.0 & 3.8 & .............. & $\ldots \ldots$ \\
\hline 10 & 3496.9 & 0.3 & 1.42 & 0.19 & 2.0 & 4.0 & SiII 1260.4: & 1.7744 \\
\hline 11 & 3515.8 & 0.3 & 1.46 & 0.20 & 1.8 & 3.7 & ............... & ....... \\
\hline 12 & 3520.8 & 0.3 & 1.16 & 0.13 & 1.8 & 5.0 & ................. & ....... \\
\hline 13 & 3531.3 & 0.3 & 1.25 & 0.22 & 2.5 & 3.5 & .............. & ....... \\
\hline 14 & 3534.0 & 0.3 & 1.89 & 0.27 & 2.5 & 3.5 & ................ & ....... \\
\hline 15 & 3542.3 & 0.3 & 1.30 & 0.25 & 1.8 & 3.3 & CI 1277.2: & 1.7735 \\
\hline 16 & 3547.2 & 0.3 & 1.33 & 0.33 & 4.0 & 3.2 & .............. & ....... \\
\hline 17 & 3551.8 & 0.3 & 2.79 & 0.27 & 5.0 & 4.1 & ............... & ....... \\
\hline 18 & 3565.0 & 0.3 & 1.63 & 0.24 & 1.5 & 3.8 & .............. & ....... \\
\hline 19 & 3576.5 & 0.3 & 2.07 & 0.16 & 3.3 & 5.9 & ............. & $\ldots \ldots$ \\
\hline 20 & 3597.0 & 0.3 & 4.13 & 0.21 & 4.5 & 5.8 & .............. & ....... \\
\hline 21 & 3602.0 & 0.3 & 1.28 & 0.15 & 2.5 & 5.0 & ……......... & ....... \\
\hline 22 & 3604.6 & 0.3 & 2.10 & 0.10 & 2.5 & 8.4 & .............. & ....... \\
\hline 23 & 3611.3 & 0.3 & 1.22 & 0.19 & 2.3 & 3.6 & OI 1302.2 & 1.7732 \\
\hline 24 & 3619.5 & 0.3 & 0.61 & 0.15 & 1.0 & 3.3 & .............. & ....... \\
\hline 25 & 3621.4 & 0.3 & 1.33 & 0.20 & 2.0 & 3.8 & ................. & ........ \\
\hline 26 & 3644.4 & 0.3 & 1.79 & 0.31 & 2.5 & 2.8 & .............. & ....... \\
\hline 27 & 3647.5 & 0.3 & 0.80 & 0.17 & 1.5 & 3.4 & ............... & ....... \\
\hline 28 & 3654.5 & 0.3 & 1.60 & 0.22 & 2.3 & 4.1 & ............. & ....... \\
\hline 29 & 3660.6 & 0.3 & 0.70 & 0.14 & 1.5 & 4.1 & ……........ & ....... \\
\hline 30 & 3662.2 & 0.3 & 1.13 & 0.13 & 1.3 & 4.2 & ............... & $\ldots \ldots$. \\
\hline 31 & 3664.3 & 0.3 & 1.23 & 0.23 & 1.8 & 3.3 & ............... & ....... \\
\hline 32 & 3676.9 & 0.3 & 1.07 & 0.27 & 3.3 & 3.6 & SiII 1304.4 & 1.8188 \\
\hline 33 & 3681.3 & 0.3 & 2.62 & 0.20 & 3.3 & 5.8 & ............... & ....... \\
\hline 34 & 3687.6 & 0.3 & 1.41 & 0.16 & 1.3 & 4.9 & ................. & ....... \\
\hline 35 & 3691.0 & 0.3 & 2.09 & 0.18 & 2.3 & 4.9 & ................ & ....... \\
\hline 36 & 3699.3 & 0.3 & 1.27 & 0.19 & 2.0 & 3.9 & ................ & ....... \\
\hline 37 & 3701.5 & 0.3 & 0.70 & 0.11 & 1.5 & 4.8 & CII 1334.5 & 1.7737 \\
\hline 38 & 3707.2 & 0.3 & 2.75 & 0.21 & 3.0 & 5.3 & ............... &.$\ldots$. \\
\hline 39 & 3734.8 & 0.3 & 1.59 & 0.18 & 1.5 & 4.1 & .............. & ........ \\
\hline 40 & 3745.7 & 0.3 & 0.97 & 0.22 & 3.8 & 4.1 & .............. & ....... \\
\hline 41 & 3750.0 & 0.3 & 1.17 & 0.16 & 1.3 & 4.7 & ………. & ....... \\
\hline 42 & 3754.0 & 0.3 & 1.89 & 0.14 & 0.8 & 5.3 & ............... & $\ldots \ldots$ \\
\hline 43 & 3755.8 & 0.3 & 0.99 & 0.11 & 1.0 & 5.6 & HI 1215.7 & 2.0894 \\
\hline 44 & 3762.3 & 0.3 & 1.18 & 0.18 & 2.0 & 5.0 & CII 1334.5 & 1.8193 \\
\hline 45 & 3800.2 & 0.3 & 0.65 & 0.11 & 1.5 & 5.0 & ................. & ....... \\
\hline 46 & 3802.8 & 0.3 & 2.30 & 0.10 & 2.5 & 8.5 & ............ & ....... \\
\hline 47 & 3811.7 & 0.3 & 1.72 & 0.16 & 2.0 & 5.5 & ................. & ....... \\
\hline 48 & 3821.4 & 0.3 & 1.31 & 0.18 & 1.8 & 6.1 & .............. & ....... \\
\hline 49 & 3826.7 & 0.3 & 1.39 & 0.17 & 3.5 & 5.9 & ............ & ....... \\
\hline 50 & 3838.5 & 0.3 & 1.26 & 0.11 & 2.0 & 6.6 & …........... & ....... \\
\hline 51 & 3850.1 & 0.3 & 0.55 & 0.09 & 1.3 & 5.7 & ………... & ....... \\
\hline 52 & 3853.4 & 0.3 & 1.16 & 0.12 & 2.3 & 6.5 & .............. & ....... \\
\hline 53 & 3861.1 & 0.3 & 0.63 & 0.10 & 1.3 & 6.4 & .............. & ....... \\
\hline 54 & 3875.6 & 0.3 & 0.53 & 0.07 & 1.0 & 7.8 & ............. & $\ldots \ldots$ \\
\hline 55 & 3883.1 & 0.3 & 1.18 & 0.11 & 1.8 & 8.6 & ……...... & ....... \\
\hline 56 & 3922.1 & 0.3 & 0.85 & 0.12 & 2.3 & 7.0 & .................. & ....... \\
\hline 57 & 3927.4 & 0.3 & 4.69 & 0.16 & 6.3 & 8.4 & ............. & ....... \\
\hline 58 & 3933.6 & 0.3 & 1.18 & 0.12 & 3.0 & 6.6 & ............... & ....... \\
\hline 59 & 3945.8 & 0.3 & 1.12 & 0.13 & 1.8 & 5.8 & ............. & $\ldots \ldots$ \\
\hline 60 & 3949.9 & 0.3 & 2.65 & 0.15 & 2.5 & 7.2 & ……......... & ....... \\
\hline 61 & 3954.6 & 0.3 & 2.63 & 0.14 & 2.3 & 7.3 & ............. & $\ldots \ldots$ \\
\hline 62 & 3964.8 & 0.3 & 1.13 & 0.11 & 1.0 & 6.8 & ............... & $\ldots \ldots$ \\
\hline 63 & 3966.8 & 0.3 & 0.88 & 0.12 & 1.0 & 5.7 & .............. & $\ldots \ldots$ \\
\hline 64 & 3970.3 & 0.3 & 0.67 & 0.12 & 2.0 & 6.0 & ............. & ....... \\
\hline 65 & 3980.1 & 0.3 & 0.70 & 0.14 & 0.8 & 6.0 & .............. & ....... \\
\hline 66 & 3999.2 & 0.3 & 0.54 & 0.12 & 0.8 & 5.8 & .............. & ....... \\
\hline 67 & 4006.3 & 0.3 & 0.79 & 0.12 & 1.0 & 5.7 & ............ & $\ldots \ldots$. \\
\hline
\end{tabular}




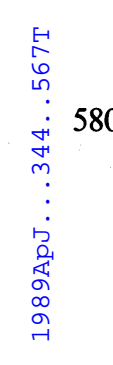

TURNSHEK $E T A L$.

Vol. 344

TABLE 4-Continued

\begin{tabular}{|c|c|c|c|c|c|c|c|c|}
\hline Line No. & $\lambda(\AA)$ & $\sigma_{\lambda}(\AA)$ & $\mathrm{E}(\AA)$ & $\sigma_{E}(\AA)$ & FWHM $(\AA)$ & $\mathrm{STN}^{a}$ & Identification & $\mathbf{z}_{a b s}$ \\
\hline 68 & 4016.1 & 0.3 & 1.80 & 0.12 & 1.8 & 6.4 & ............ & ...... \\
\hline 69 & 4022.9 & 0.3 & 5.77 & 0.14 & 6.0 & 11.0 & ............. & $\ldots . .$. \\
\hline 70 & 4043.4 & 0.3 & 0.67 & 0.08 & 1.0 & 6.1 & $\ldots \ldots \ldots \ldots$ & $\ldots \ldots$ \\
\hline 71 & 4049.9 & 0.3 & 1.32 & 0.13 & 2.5 & 6.3 & ............. & ...... \\
\hline 72 & 4054.9 & 0.3 & 3.49 & 0.14 & 3.5 & 8.4 & ............ & $\ldots .$. \\
\hline 73 & 4061.9 & 0.3 & 1.08 & 0.13 & 1.5 & 6.9 & ............. & $\ldots . .$. \\
\hline 74 & 4066.5 & 0.3 & 1.14 & 0.12 & 1.5 & 6.1 & .............. & ....... \\
\hline 75 & 4068.8 & 0.3 & 0.82 & 0.10 & 1.5 & 5.7 & $\ldots \ldots \ldots \ldots$ & $\ldots \ldots$ \\
\hline 76 & 4071.2 & 0.3 & 2.02 & 0.18 & 4.0 & 6.4 & $\ldots \ldots \ldots \ldots$ & $\ldots \ldots$ \\
\hline 77 & 4076.5 & 0.3 & 1.36 & 0.11 & 1.5 & 7.3 & $\ldots \ldots \ldots .$. & $\ldots \ldots$ \\
\hline 78 & 4085.1 & 0.3 & 0.47 & 0.11 & 1.8 & 6.6 & $\ldots \ldots \ldots$ & $\ldots \ldots$ \\
\hline 79 & 4092.3 & 0.3 & 4.47 & 0.13 & 5.0 & 10.1 & …......... & $\ldots \ldots$ \\
\hline 80 & 4097.9 & 0.3 & 2.17 & 0.16 & 2.0 & 7.6 & ............ & $\cdots \cdots$ \\
\hline 81 & 4166.8 & 0.4 & 6.37 & 0.23 & 10.5 & 7.0 & ............. & ...... \\
\hline 82 & 4176.8 & 0.3 & 1.13 & 0.16 & 3.3 & 5.6 & $\ldots \ldots \ldots \cdots$ & ...... \\
\hline 83 & 4188.5 & 0.3 & 4.60 & 0.20 & 5.0 & 6.3 & ............ & $\ldots \ldots$ \\
\hline 84 & 4199.8 & 0.3 & 2.59 & 0.14 & 3.0 & 6.4 & $\cdots \cdots \cdots \cdots$ & ...... \\
\hline 85 & 4209.2 & 0.3 & 0.83 & 0.14 & 1.3 & 5.5 & ............. & ...... \\
\hline 86 & 4220.3 & 0.3 & 0.78 & 0.13 & 1.3 & 5.4 & …......... & ...... \\
\hline 87 & 4227.0 & 0.3 & 1.79 & 0.14 & 2.5 & 6.2 & ............ & $\ldots \ldots$ \\
\hline 88 & 4234.9 & 0.3 & 0.81 & 0.11 & 2.0 & 5.5 & SiII 1526.7 & 1.7739 \\
\hline 89 & 4240.3 & 0.3 & 0.88 & 0.13 & 2.0 & 5.7 & ............ & $\ldots \ldots$ \\
\hline 90 & 4243.9 & 0.3 & 1.44 & 0.15 & 1.0 & 5.9 & ............ & $\ldots \ldots$ \\
\hline 91 & 4248.8 & 0.3 & 0.74 & 0.11 & 1.3 & 5.2 & 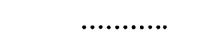 & $\cdots \cdots$ \\
\hline 92 & 4253.6 & 0.3 & 1.54 & 0.18 & 3.5 & 5.7 & ............ & ...... \\
\hline 93 & 4269.8 & 0.3 & 1.81 & 0.14 & 2.0 & 5.8 & ............ & $\ldots \ldots$. \\
\hline 94 & 4279.8 & 0.3 & 0.64 & 0.13 & 0.5 & 5.3 & ............. & $\ldots \ldots$ \\
\hline 95 & 4295.7 & 0.3 & 1.88 & 0.16 & 1.5 & 6.0 & ............ & $\ldots \ldots$ \\
\hline 96 & 4304.7 & 0.3 & 2.71 & 0.17 & 3.5 & 6.6 & ............. & $\ldots \ldots$ \\
\hline 97 & 4315.8 & 0.3 & 0.58 & 0.09 & 1.0 & 5.4 & ............. & $\ldots \ldots$ \\
\hline 98 & 4319.0 & 0.3 & 0.86 & 0.11 & 1.3 & 5.3 & ............ & $\ldots \ldots$ \\
\hline 99 & 4323.8 & 0.3 & 1.56 & 0.14 & 2.0 & 6.1 & …......... & $\cdots \cdots$ \\
\hline 100 & 4331.9 & 0.3 & 2.25 & 0.16 & 4.0 & 6.1 & ............ & $\ldots \ldots$ \\
\hline 101 & 4344.3 & 0.3 & 2.41 & 0.16 & 3.0 & 6.2 & …........ & $\ldots \ldots$ \\
\hline 102 & 4351.0 & 0.3 & 0.82 & 0.11 & 1.5 & 5.3 & ............ & $\ldots \ldots$ \\
\hline 103 & 4356.3 & 0.3 & 2.12 & 0.15 & 2.0 & 6.1 & ............ & $\ldots \ldots$ \\
\hline 104 & 4364.0 & 0.3 & 4.03 & 0.20 & 5.5 & 5.8 & CIV 1548.2 & 1.8188 \\
\hline 105 & 4371.2 & 0.3 & 3.41 & 0.16 & 4.3 & 6.4 & CIV 1550.8 & 1.8187 \\
\hline 106 & 4376.5 & 0.3 & 1.86 & 0.17 & 4.5 & 6.1 & ............. & ...... \\
\hline 107 & 4384.9 & 0.3 & 2.62 & 0.16 & 2.5 & 6.6 & ............. & $\ldots \ldots$. \\
\hline 108 & 4399.8 & 0.3 & 2.08 & 0.13 & 2.0 & 6.5 & ............ & $\ldots \ldots$ \\
\hline 109 & 4433.4 & 0.3 & 0.75 & 0.15 & 3.3 & 5.9 & ............ & $\ldots \ldots$ \\
\hline 110 & 4439.3 & 0.3 & 3.98 & 0.14 & 4.8 & 7.3 & ........... & $\ldots \ldots$ \\
\hline 111 & 4444.0 & 0.3 & 2.84 & 0.13 & 3.3 & 7.0 & ............. & $\ldots \ldots$ \\
\hline 112 & 4461.4 & 0.3 & 0.94 & 0.11 & 1.8 & 6.6 & FeII 1608.5 & 1.7736 \\
\hline 113 & 4467.3 & 0.3 & 2.23 & 0.11 & 4.0 & 7.0 & ............ & $\ldots \ldots$ \\
\hline 114 & 4469.4 & 0.3 & 1.36 & 0.08 & 1.5 & 6.9 & ............. & $\ldots \ldots$ \\
\hline 115 & 4472.3 & 0.3 & 1.64 & 0.13 & 2.0 & 6.9 & ............ & $\ldots \ldots$ \\
\hline 116 & 4476.9 & 0.3 & 0.37 & 0.08 & 1.3 & 5.6 & ............ & $\ldots \ldots$ \\
\hline 117 & 4484.4 & 0.3 & 0.54 & 0.11 & 1.3 & 6.6 & …........ & $\ldots \ldots$ \\
\hline 118 & 4491.1 & 0.3 & 1.43 & 0.10 & 1.5 & 7.4 & ............ & $\cdots \cdots$ \\
\hline 119 & 4503.5 & 0.3 & 1.35 & 0.07 & 1.5 & 8.4 & ............ & $\ldots \ldots$. \\
\hline 120 & 4506.8 & 0.3 & 4.14 & 0.11 & 4.0 & 9.9 & ............ & $\ldots \ldots$ \\
\hline 121 & 4533.6 & 0.3 & 0.76 & 0.08 & 2.5 & 8.9 & FeII 1608.5 & 1.8185 \\
\hline 122 & 4536.1 & 0.3 & 0.47 & 0.06 & 1.3 & 8.9 & $\ldots \ldots \ldots$ & $\ldots \ldots$ \\
\hline 123 & 4545.5 & 0.3 & 0.46 & 0.05 & 1.5 & 11.1 & ............. & $\ldots \ldots$ \\
\hline 124 & 4548.3 & 0.3 & 0.97 & 0.06 & 1.5 & 13.0 & ............ & $\ldots \ldots$ \\
\hline 125 & 4561.1 & 0.3 & 0.60 & 0.04 & 1.3 & 14.4 & ............ & $\ldots \ldots$ \\
\hline 126 & 4565.6 & 0.3 & 0.80 & 0.04 & 1.5 & 17.7 & $\ldots \ldots \ldots$ & $\ldots \ldots$ \\
\hline 127 & 4581.5 & 0.3 & 1.00 & 0.06 & 1.5 & 13.3 & CIV 1548.2 & 1.9592 \\
\hline 128 & 4588.9 & 0.3 & 0.41 & 0.05 & 1.0 & 10.8 & CIV 1550.8 & 1.9591 \\
\hline 129 & 4634.7 & 0.3 & 1.30 & 0.11 & 1.5 & 7.4 & AlII 1670.8 & 1.7739 \\
\hline 130 & 4648.8 & 0.3 & 0.52 & 0.09 & 1.8 & 7.2 & ............. & $\ldots \ldots$ \\
\hline 131 & 4652.5 & 0.3 & 0.46 & 0.07 & 1.3 & 6.1 & ........... & $\ldots \ldots$ \\
\hline 132 & 4678.8 & 0.3 & 0.52 & 0.11 & 1.8 & 6.2 & ............ & $\ldots \ldots$ \\
\hline 133 & 4691.4 & 0.3 & 0.60 & 0.10 & 1.5 & 5.6 & ............ & $\ldots \ldots$ \\
\hline
\end{tabular}


TABLE 4-Continued

\begin{tabular}{|c|c|c|c|c|c|c|c|c|}
\hline Line No. & $\lambda(\AA)$ & $\sigma_{\lambda}(\AA)$ & $\mathrm{E}(\AA)$ & $\sigma_{E}(\AA)$ & FWHM $(\AA)$ & $\mathrm{STN}^{a}$ & Identification & $\mathbf{z}_{a b s}$ \\
\hline 134 & 4710.2 & 0.3 & 0.75 & 0.13 & 2.0 & 5.1 & $\begin{array}{r}\text { MgII } 2796.4 \\
\text { AlII } 1670.8\end{array}$ & $\begin{array}{l}0.6844 \\
1.8191\end{array}$ \\
\hline 135 & 4721.5 & 0.3 & 0.59 & 0.13 & 0.5 & 4.8 & MgII 2803.5 & 0.6841 \\
\hline 136 & 4782.8 & 0.3 & 0.74 & 0.15 & 1.3 & 4.3 & CIV 1548.2 & 2.0893 \\
\hline 137 & 4791.0 & 0.3 & 0.78 & 0.14 & 1.0 & 4.1 & CIV 1550.8 & 2.0894 \\
\hline 138 & 6502.5 & 0.2 & 1.43 & 0.11 & 3.0 & 32.3 & FeII 2344.2 & 1.7738 \\
\hline 139 & 6586.2 & 0.2 & 1.12 & 0.09 & 3.0 & 35.6 & FeII 2374.5 & 1.7738 \\
\hline 140 & 6609.3 & 0.2 & 1.37 & 0.08 & 2.4 & 36.7 & FeII 2382.8 & 1.7738 \\
\hline 141 & 6718.1 & 0.3 & 0.60 & 0.08 & 2.7 & 36.2 & FeII 2382.8 & 1.8195 \\
\hline 142 & 7175.2 & 0.2 & 0.98 & 0.08 & 2.7 & 37.7 & FeII 2586.7 & 1.7739 \\
\hline 143 & 7212.8 & 0.2 & 1.25 & 0.09 & 3.0 & 35.2 & FeII 2600.2 & 1.7740 \\
\hline 144 & 7755.0 & 0.2 & 1.43 & 0.14 & 3.0 & 27.8 & MgII 2796.4 & 1.7732 \\
\hline 145 & 7774.9 & 0.3 & 1.54 & 0.12 & 3.9 & 28.4 & MgII 2803.5 & 1.7732 \\
\hline 146 & 7824.4 & 0.3 & 0.92 & 0.15 & 2.4 & 28.1 & $\ldots \ldots \ldots$ & $\ldots \ldots$ \\
\hline
\end{tabular}

NOTES ON INDIVIDUAL LINES

Line 10.-Wavelength agreement is poor; this is probably a Ly $\alpha$ forest line.

Line 15. - This identification is probably incorrect, since the line lies in the Ly $\alpha$ forest and the line C I $\lambda 1656.9$, which lies outside of the Ly $\alpha$ forest, is not seen.

Lines 127 and 128. - This identification is based only on C IV $\lambda \lambda 1548,1550$ in the Ly $\alpha$ forest.

Lines 134 and 135.- This identification is based only on $\mathrm{Mg}$ II $\lambda \lambda 2796,2803$, but the lines lie outside the Ly $\alpha$ forest.

a Signal-to-noise ratio of the continuum spectrum, which includes the fit to candidate damped Ly $\alpha$, evaluated at $\lambda$.

confused at this resolution ( $2 \AA$ FWHM) by blending with an absorption line due to $\mathrm{Fe}$ II 22585 at $z_{\mathrm{abs}}=0.7874$; however, it was included in the curve-of-growth analysis because this will have no effect on the determination of $\sigma$. The Si II $\lambda 1260.4$ line at $z_{\text {abs }}=2.4660$ may be blended with a C IV doublet (see Table 2); however, this C IV system is based only on two lines and may be erroneous.

$$
\text { ii) The } Q 1136+122 z_{\text {abs }}=1.7890 \text { System }
$$

The strong Ly $\alpha$ absorption feature in this system is fairly well fitted by a damping profile, despite the relatively poor signal-to-noise ratio in the near-ultraviolet parts of the spectrum. Metal line absorption from $\mathrm{C}$ II, $\mathrm{Si}$ II, and $\mathrm{Si}$ IV is not detected in this system; however, these lines are predicted to lie

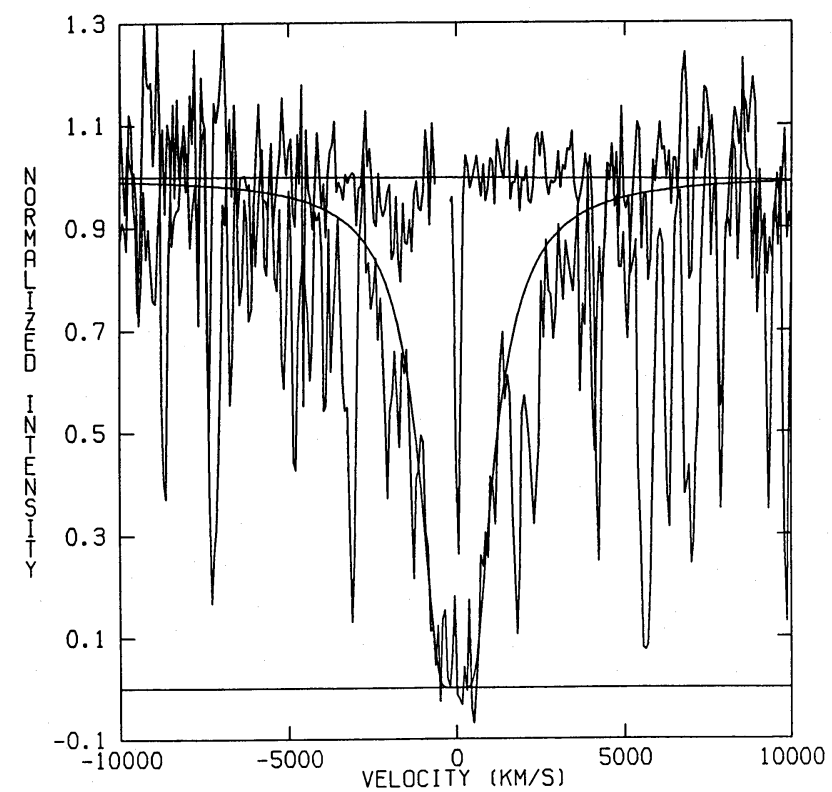

FIG. 7.-Velocity profile of the $\mathrm{O}$ I line in the Q0836+113 $z_{\text {abs }}=2.4660$ system superposed on the inferred velocity profile of the $\mathrm{H}_{\mathrm{I}}$ at the same redshift. in the Ly $\alpha$ forest. The wavelength range observed does not include the predicted position of the $\mathrm{C}$ IV absorption doublet. Only a single $\mathrm{Fe}$ II absorption line is detected in this system at red wavelengths. Through comparison with this single detected metal line, the damped nature of the Ly $\alpha$ line is further indicated by the values $v_{\text {diff }}<60 \mathrm{~km} \mathrm{~s}^{-1}$ and $\Delta v_{\text {metals }}<110 \mathrm{~km}$ $\mathrm{s}^{-1}$, while $\Delta v_{\mathrm{HI}} \simeq 1200 \mathrm{~km} \mathrm{~s}^{-1}$. The inferred neutral hydrogen column density based on the Voigt profile fit to Ly $\alpha$ gives $N(\mathrm{H} \mathrm{I}) \simeq 1 \times 10^{20} \mathrm{~cm}^{-2}$. This falls below the $N\left(\mathrm{H}_{\mathrm{I}}\right) \geq 2 \times 10^{20} \mathrm{~cm}^{-2}$ selection limit discussed in Paper I. Because of the lack of data, a curve-of-growth analysis could not be performed for this system; however, see $\S$ IV $d$ for additional discussion.

ii) The $Q 1151+068 z_{\mathrm{abs}}=1.7737$ System

The signal-to-noise ratio is poor in the near-ultraviolet part of the spectrum where the Ly $\alpha$ feature occurs. The large depression centered near $3370 \AA$ can be fitted with a damped Ly $\alpha$ profile having $z_{\text {abs }}=1.781$ and $N(\mathrm{H} \mathrm{I})=4 \times 10^{21} \mathrm{~cm}^{-2}$. However, this yields the values $v_{\text {diff }} \simeq 800 \mathrm{~km} \mathrm{~s}^{-1}$ and $\Delta v_{\text {metals }}<110 \mathrm{~km} \mathrm{~s}^{-1}$, while $\Delta v_{\mathrm{H} \mathrm{I}} \simeq 7800 \mathrm{~km} \mathrm{~s}^{-1}$. Therefore, if the column density is this large, two interpretations are possible. One possibility is that $v_{\text {diff }}$ is measured to be large because the near-ultraviolet wavelength calibration is poorly determined. We consider this possibility unlikely. The other possibility is that the damped system is largely devoid of detectable heavy elements such as Fe II and $\mathrm{Mg}$ II. If this is the case, one would have to suppose that the nearby system with $z_{\text {abs }}=1.7737$ chanced to be present. On the other hand, the redshift of the damped Ly $\alpha$ line may correspond to $z_{\text {abs }}=$ 1.7737 , the redshift determined by the metal lines. In this case, the column density is set by a fit to the short-wavelength wing of the Ly $\alpha$ profile, $N(\mathrm{H} \mathrm{I}) \simeq 1.8 \times 10^{21} \mathrm{~cm}^{-2}$. Absorption on the longward side of the damped Ly $\alpha$ profile is not due to the damping wings, and $\Delta v_{\mathrm{HI}}$ is $\sim 5200 \mathrm{~km} \mathrm{~s}^{-1}$. The presence of the $\mathrm{C}$ IV system at $z_{\text {abs }}=1.8187$ undoubtedly does cause some Ly $\alpha$ absorption on the longward side of the damping profile. We believe that this is the more plausible interpretation and adopt it in the remaining discussion.

The system is then found to be characterized by unresolved 
TABLE 5

ABSORPTION-LINE LIST FOR Q1337+113

\begin{tabular}{|c|c|c|c|c|c|c|c|c|}
\hline Line No. & $\lambda(\AA)$ & $\sigma_{\lambda}(\AA)$ & $\mathrm{E}(\AA)$ & $\sigma_{E}(\AA)$ & FWHM $(\AA)$ & $\mathrm{STN}^{a}$ & Identification & $\mathrm{z}_{a b s}$ \\
\hline 1 & 4485.1 & 0.5 & 1.07 & 0.16 & 5.0 & 10.4 & .................... & ....... \\
\hline 2 & 4490.0 & 0.7 & 0.62 & 0.15 & 1.5 & 10.2 & ….............. & $\ldots \ldots$. \\
\hline 3 & 4498.7 & 0.5 & 1.04 & 0.11 & 2.5 & 10.0 & ............. & ....... \\
\hline 4 & 4505.4 & 0.6 & 4.34 & 0.18 & 4.0 & 11.9 & …............... & ....... \\
\hline 5 & 4511.4 & 0.6 & 0.53 & 0.11 & 2.0 & 9.5 & ................... & ....... \\
\hline 6 & 4519.0 & 0.6 & 1.02 & 0.13 & 2.5 & 10.5 & SiII 1190.4 & 2.7963 \\
\hline 7 & 4528.9 & 0.6 & 0.64 & 0.11 & 2.0 & 9.3 & SiII 1193.3 & 2.7953 \\
\hline 8 & 4539.7 & 0.6 & 0.90 & 0.14 & 2.5 & 9.9 & ................... & $\ldots . .$. \\
\hline 9 & 4549.4 & 0.5 & 1.27 & 0.17 & 1.5 & 10.1 & …............. & $\ldots \ldots$. \\
\hline 10 & 4556.1 & 0.5 & 0.50 & 0.12 & 2.0 & 8.9 & ….............. & ....... \\
\hline 11 & 4562.0 & 0.6 & 0.95 & 0.13 & 3.0 & 9.4 & ................. & $\ldots \ldots$. \\
\hline 12 & 4655.9 & 0.5 & 0.59 & 0.11 & 2.0 & 10.4 & ............... & $\ldots \ldots$ \\
\hline 13 & 4661.1 & 0.5 & 1.95 & 0.12 & 2.5 & 12.2 & CIV 1548.2 & 2.0107 \\
\hline \multirow[t]{2}{*}{14} & 4669.0 & 0.5 & 1.06 & 0.10 & 2.0 & 13.0 & CIV 1550.8 & 2.0107 \\
\hline & & & & & & & CIV 1548.2 & 2.0158 \\
\hline 15 & 4676.7 & 0.5 & 0.68 & 0.09 & 1.5 & 13.9 & CIV 1550.8 & 2.0157 \\
\hline 16 & 4693.0 & 0.5 & 5.36 & 0.10 & 6.0 & 20.5 & .................. & $\ldots \ldots$ \\
\hline 17 & 4720.4 & 0.5 & 4.24 & 0.07 & 6.0 & 28.3 & ............ & $\ldots \ldots$ \\
\hline 18 & 4739.3 & 0.5 & 2.19 & 0.05 & 3.5 & 33.0 & ................. & $\ldots \ldots$. \\
\hline 19 & 4751.1 & 0.5 & 1.25 & 0.04 & 2.5 & 42.2 & ................... & $\ldots \ldots$ \\
\hline 20 & 4759.1 & 0.6 & 0.28 & 0.03 & 2.0 & 43.9 & .............. & $\ldots \ldots$. \\
\hline 21 & 4784.1 & 0.5 & 0.80 & 0.04 & 2.5 & 35.1 & SiII 1260.4 & 2.7957 \\
\hline 22 & 4809.1 & 0.6 & 0.28 & 0.05 & 3.0 & 27.4 & & ....... \\
\hline 23 & 4860.4 & 0.6 & 3.33 & 0.06 & 4.0 & 31.1 & CIV 1548.2 & 2.1394 \\
\hline 24 & 4868.3 & 0.5 & 2.28 & 0.06 & 7.0 & 29.0 & CIV 1550.2 & 2.1392 \\
\hline 25 & 4877.0 & 0.5 & 1.22 & 0.07 & 3.5 & 25.9 & CIV 1548.2 & 2.1501 \\
\hline 26 & 4884.7 & 0.5 & 0.91 & 0.05 & 2.5 & 22.7 & CIV 1550.8 & 2.1498 \\
\hline 27 & 4889.0 & 0.6 & 1.15 & 0.07 & 3.0 & 23.5 & SiIV 1393.8 & 2.5077 \\
\hline 28 & 4920.4 & 0.5 & 1.09 & 0.07 & 2.5 & 20.6 & SiIV 1402.8 & 2.5076 \\
\hline 29 & 4942.8 & 0.5 & 1.08 & 0.10 & 3.0 & 19.9 & OI 1302.2 & 2.7957 \\
\hline 30 & 4951.2 & 0.5 & 0.68 & 0.08 & 2.5 & 18.2 & SiII 1304.4 & 2.7958 \\
\hline 31 & 5065.6 & 0.5 & 1.24 & 0.10 & 2.5 & 17.0 & CII 1334.5 & 2.7959 \\
\hline 32 & 5187.1 & 0.5 & 0.89 & 0.10 & 2.5 & 16.1 & & ....... \\
\hline ** & 5793.6 & 1.2 & 1.04 & 0.31 & 7.8 & 12.2 & SiII 1526.7 & 2.7948 \\
\hline 33 & 5975.1 & 0.5 & 1.62 & 0.27 & 5.1 & 15.9 & $\ldots . . . . . .$. & $\ldots \ldots$ \\
\hline ** & 6104.7 & 0.5 & 0.63 & 0.16 & 3.9 & 21.6 & FeII 1608.5 & 2.7954 \\
\hline 34 & 6281.4 & 0.2 & 1.55 & 0.21 & 2.7 & 15.5 & ……...... & $\ldots \ldots$ \\
\hline$* *$ & 6340.3 & 0.7 & 0.66 & 0.21 & 4.5 & 16.1 & AlII 1670.8 & 2.7955 \\
\hline
\end{tabular}

NOTES ON INDIVIDUAL LINES

Lines 13 and $14 ; 14$ and 15 .- These identifications are based only on C IV $\lambda \lambda 1548,1550$ in the Ly $\alpha$ forest.

Lines 23 and $24 ; 25$ and 26 . - These identifications are based only on C IV $\lambda \lambda 1548,1550$, but the lines lie outside the Ly $\alpha$ forest.

Lines 27 and 28.-This identification is based only on Si IV $\lambda \lambda 1393,1403$, but the lines lie outside the Ly $\alpha$ forest.

a Signal-to-noise ratio of the continuum spectrum, which includes the fit to candidate damped Ly $\alpha$, evaluated at $\lambda$.

low-ion Fe II and Mg II absorption and weak or absent highion absorption. The curve-of-growth analysis, illustrated in Figure 9 and based on seven Fe II lines and two $\mathrm{Mg}$ II lines, gives $\sigma=8.5 \mathrm{~km} \mathrm{~s}^{-1}$. This system appears to be a pure quiescent component without an ionized or turbulent component. See $\S$ IV $d$ for a discussion of constraints on abundances in this system.

$$
\text { iv) The } Q 1337+113 z_{\text {abs }}=2.7957 \text { System }
$$

The damped nature of the strong Ly $\alpha$ absorption feature near $4600 \AA$ is supported by an excellent fit to the damped Ly $\alpha$ profile, with $N(\mathrm{H} \mathrm{I}) \simeq 8 \times 10^{20}$. The Ly $\beta$ and Ly $\gamma$ profiles are consistent with this. Only weak low-ion metal lines are present in the spectrum. The fact that $v_{\text {diff }}<50 \mathrm{~km} \mathrm{~s}^{-1}$ and $\Delta v_{\text {metals }}<$ $120 \mathrm{~km} \mathrm{~s}^{-1}$, while $\Delta v_{\mathrm{HI}} \simeq 3500 \mathrm{~km} \mathrm{~s}^{-1}$, provides further evidence that the Ly $\alpha$ absorption feature is damped. The small value of $v_{\text {diff }}$ is illustrated in Figure 10 , where the velocity profile of $\mathrm{C}$ II and the inferred velocity profile of $\mathrm{H}$ I are superposed. A curve-of-growth analysis, illustrated in Figure 11 and based on six $\mathrm{Si}$ II lines and nine $\mathrm{Fe}$ II lines, indicates that $\sigma \simeq 8.3 \mathrm{~km} \mathrm{~s}^{-1}$. Again, the combination of low effective velocity dispersion and an absence of high-ion absorption suggest that this system is a pure quiescent component without an ionized or turbulent component. A curve-of-growth analysis, which uses $\sigma=8.3 \mathrm{~km} \mathrm{~s}^{-1}$ and assumes that the $3 \sigma$ upper limit on the $\mathrm{C}$ IV equivalent width lies on the linear part of the curve of growth, suggests that $N\left(\mathrm{C}^{+}\right) / N\left(\mathrm{C}^{+3}\right)>10^{3}$. Note that the fits to $\operatorname{Ly} \beta$ and $\operatorname{Ly} \gamma$ absorption lines in the $z_{\mathrm{abs}}=2.7957$ system are shown in the spectrum presented in Lanzetta, Wolfe, and Turnshek (1989). The measurements of one of the $\mathrm{Si}$ II lines and seven of the Fe II lines used in the curve-ofgrowth analysis are also presented there. See $\S$ IV $d$ for a discussion of constraints on abundances in this system.

$$
\text { v) The } Q 1347+112 z_{\mathrm{abs}}=2.4709 \text { System }
$$

The strong $\mathrm{Ly} \alpha$ absorption feature in this system is well fitted by a damping profile with $N(\mathrm{H} \mathrm{I}) \simeq 2 \times 10^{20} \mathrm{~cm}^{-2}$. The system is characterized by strong, resolved low- and high-ion absorption. Additional evidence indicating damping is that $v_{\text {diff }}<50 \mathrm{~km} \mathrm{~s}^{-1}$ and $\Delta v_{\text {metals }} \simeq 400 \mathrm{~km} \mathrm{~s}^{-1}$, even though 
TABLE 6

ABSORPTION-LINE LIST FOR Q1347+ 112

\begin{tabular}{|c|c|c|c|c|c|c|c|c|}
\hline Line No. & $\lambda(\AA)$ & $\sigma_{\lambda}(\AA)$ & $E(\AA)$ & $\sigma_{E}(\AA)$ & FWHM $(\AA)$ & STN $^{a}$ & Identification & $\mathbf{z}_{a b s}$ \\
\hline 1 & 4132.2 & 0.6 & 5.50 & 0.29 & 5.0 & 7.9 & SiII 1190.4 & 2.4713 \\
\hline \multirow[t]{2}{*}{2} & 4141.5 & 0.6 & 5.50 & 0.26 & 6.5 & 7.6 & SiII 1193.3 & 2.4706 \\
\hline & & & & & & & FeII 2586.7: & 0.6011 \\
\hline 3 & 4148.0 & 0.6 & 1.57 & 0.18 & 4.5 & 9.3 & $\ldots \ldots \ldots$ & $\ldots \ldots$ \\
\hline 4 & 4160.5 & 0.6 & 2.96 & 0.24 & 5.0 & 7.9 & ….......... & $\ldots \ldots$ \\
\hline 5 & 4165.8 & 0.7 & 2.81 & 0.22 & 5.5 & 8.5 & FeII 2600.2: & 0.6021 \\
\hline 6 & 4188.5 & 0.6 & 6.21 & 0.28 & 5.5 & 8.6 & SiIII 1206.5 & 2.4716 \\
\hline 7 & 4246.3 & 0.6 & 1.90 & 0.23 & 2.5 & 6.5 & ............. & $\ldots \ldots$ \\
\hline 8 & 4270.5 & 0.6 & 0.97 & 0.18 & 3.5 & 7.8 & ............ & $\ldots \ldots$ \\
\hline 9 & 4275.7 & 0.6 & 0.95 & 0.23 & 1.5 & 7.5 & ............ & $\ldots \ldots$ \\
\hline 10 & 4286.9 & 0.6 & 1.08 & 0.24 & 5.0 & 7.2 & ............... & $\ldots \ldots$ \\
\hline 11 & 4310.7 & 0.6 & 1.85 & 0.22 & 3.5 & 7.3 & SiII 1190.4 & 2.6212 \\
\hline 12 & 4320.5 & 0.6 & 2.50 & 0.32 & 5.5 & 5.7 & SiII 1193.3 & 2.6206 \\
\hline 13 & 4341.0 & 0.6 & 0.71 & 0.17 & 2.5 & 6.6 & ............ & $\ldots \ldots$ \\
\hline 14 & 4350.0 & 0.7 & 1.60 & 0.23 & 4.5 & 7.8 & ............. & ...... \\
\hline 15 & 4369.1 & 0.6 & 4.43 & 0.21 & 5.5 & 9.7 & SiIII 1206.5 & 2.6213 \\
\hline 16 & 4375.2 & 0.6 & 4.56 & 0.25 & 5.5 & 7.1 & SiII 1260.4 & 2.4713 \\
\hline 17 & 4384.7 & 0.6 & 4.79 & 0.34 & 4.5 & 6.9 & ............. & ....... \\
\hline 18 & 4401.8 & 0.6 & 6.45 & 0.27 & 7.5 & 7.3 & HI 1215.7 & 2.6208 \\
\hline 19 & 4408.7 & 0.7 & 6.56 & 0.29 & 9.0 & 7.7 & .............. & ...... \\
\hline 20 & 4419.6 & 0.6 & 3.04 & 0.21 & 4.0 & 8.6 & …......... & ...... \\
\hline 21 & 4443.1 & 0.6 & 0.93 & 0.11 & 5.5 & 14.4 & ............. & $\ldots \ldots$ \\
\hline 22 & 4483.8 & 0.6 & 6.94 & 0.12 & 8.0 & 20.9 & MgII 2796.4 & 0.6034 \\
\hline 23 & 4496.3 & 0.9 & 3.17 & 0.09 & 5.5 & 18.1 & MgII 2803.5 & 0.6038 \\
\hline 24 & 4519.1 & 0.6 & 3.85 & 0.14 & 3.5 & 14.8 & OI 1302.2 & 2.4704 \\
\hline 25 & 4526.9 & 0.6 & 3.31 & 0.13 & 3.5 & 13.7 & SiII 1304.4 & 2.4705 \\
\hline 26 & 4563.6 & 0.6 & 2.32 & 0.15 & 4.0 & 11.6 & SiII 1260.4 & 2.6208 \\
\hline 27 & 4632.8 & 0.6 & 5.79 & 0.27 & 5.5 & 8.5 & CII 1334.5 & 2.4716 \\
\hline 28 & 4831.9 & 0.6 & 2.77 & 0.28 & 3.5 & 6.4 & CII 1334.5 & 2.6208 \\
\hline 29 & 4838.0 & 0.6 & 3.08 & 0.32 & 6.0 & 5.9 & SiIV 1393.8 & 2.4711 \\
\hline 30 & 4868.5 & 0.6 & 1.75 & 0.34 & 1.5 & 5.5 & SiIV 1402.8 & 2.4706 \\
\hline 31 & 5046.9 & 0.6 & 2.83 & 0.38 & 3.5 & 4.7 & SiIV 1393.8 & 2.6210 \\
\hline 32 & 5079.5 & 0.6 & 2.50 & 0.41 & 2.5 & 5.0 & SiIV 1402.8 & 2.6210 \\
\hline 33 & 5298.5 & 0.6 & 3.86 & 0.48 & 3.5 & 3.7 & SiII 1526.7 & 2.4706 \\
\hline 34 & 5374.4 & 0.6 & 6.22 & 0.57 & 7.0 & 4.1 & CIV 1548.2 & 2.4714 \\
\hline 35 & 5382.9 & 0.6 & 2.32 & 0.45 & 5.5 & 3.8 & CIV 1550.8 & 2.4710 \\
\hline 36 & 5581.2 & 0.6 & 6.24 & 0.58 & 7.0 & 3.5 & FeII 1608.5 & 2.4698 \\
\hline 37 & 5605.9 & 0.6 & 3.13 & 0.42 & 4.0 & 3.6 & CIV 1548.2 & 2.6209 \\
\hline 38 & 5614.9 & 0.6 & 3.01 & 0.45 & 4.0 & 3.3 & CIV 1550.8 & 2.6206 \\
\hline 39 & 5793.8 & 0.6 & 2.85 & 0.53 & 5.0 & 2.8 & CIV 1548.2 & 2.7423 \\
\hline 40 & 5800.4 & 0.6 & 4.25 & 0.74 & 6.0 & 2.8 & AlII 1670.8 & 2.4716 \\
\hline & & & & & & & CIV 1550.8 & 2.7403 \\
\hline
\end{tabular}

NOTES ON INDIVIDUAL LINES

Lines 2 and 5.- These $\mathrm{Fe}$ lines were identified based on the $\mathrm{Mg}$ II redshift from lines 22 and 23 . The wavelength agreement may be poor because the lines are near the end of the spectrum. Note that if Fe II $\lambda 2686$ does contribute to line 2 , this may explain the poor agreement in redshift between lines 1 and 2 . It would also indicate that the equivalent width for the Si II $\lambda 1193$ line has been overestimated in the curve-of-growth analysis.

Lines 22 and 23.-Discounting the proposed identification of lines 2 and 5, this identification would be based solely on Mg II $\lambda \lambda 2796,2803$. An asymmetric blue wing appears to be present only on line 22 .

Lines 39 and 40 . - The identification of line 39 as $\mathrm{C}$ IV $\lambda 1548$ is based on assuming that line 40 is a blend of $\mathrm{Al}$ II $\lambda 1670$ and $\mathrm{C}$ IV $\lambda 1550$. The fact that line $\mathbf{4 0}$ is broad may support this interpretation. Moreover, this may explain why the redshifts listed for line 40 are discrepant.

a Signal-to-noise ratio of the continuum spectrum, which includes the fit to candidate damped Ly $\alpha$, evaluated at $\lambda$.

Notes ON INDIVIDUAL LiNES

Lines 7 and 8. - These lines are blended, and Si II $\lambda 1190$ is probably part of the blend. Most likely Si II $\lambda 1190$ can be identified with line 7, and this is the assumption made for the curve-of-growth analysis.

Line 20.-This line is probably blended with other features.

Line 26. - This line has an asymmetric wing on the blue side which is probably not due to Si II $\lambda 1304$. Therefore, the equivalent width of the Si II $\lambda 1304$ line may be smaller than the measurement indicates.

Line 30.-This line is probably blended with other features.

Line 47.-Si IV $\lambda 1393$ may be a part of this feature; however, Si IV $\lambda 1402$ at this redshift is not seen.

Lines 57 and 58.-This may in fact be only one feature.

a Signal-to-noise ratio of the continuum spectrum, which includes the fits to candidate damped Ly $\alpha$, evaluated at $\lambda$. 
TABLE 7

ABSORPTION-LINE LIST FOR Q2348 - 011

\begin{tabular}{|c|c|c|c|c|c|c|c|c|}
\hline Line No. & $\lambda(\AA)$ & $\sigma_{\lambda}(\AA)$ & $\mathrm{E}(\AA)$ & $\sigma_{E}(\AA)$ & FWHM $(\AA)$ & $\operatorname{STN}^{a}$ & Identification & $\mathrm{z}_{a b s}$ \\
\hline 1 & 4002.0 & 0.5 & 2.15 & 0.34 & 1.5 & 4.7 & ............ & ....... \\
\hline 2 & 4022.6 & 0.5 & 2.27 & 0.33 & 3.5 & 4.6 & ............. & ....... \\
\hline 3 & 4031.9 & 0.5 & 4.94 & 0.42 & 8.0 & 5.1 & ............ & ....... \\
\hline 4 & 4042.6 & 0.5 & 1.36 & 0.29 & 3.0 & 4.3 & ............ & ....... \\
\hline 5 & 4057.7 & 0.5 & 2.30 & 0.37 & 5.0 & 4.6 & FeII 1122.0: & 2.6165 \\
\hline 6 & 4067.3 & 0.5 & 1.93 & 0.34 & 3.0 & 4.5 & ............ & ...... \\
\hline 7 & 4077.7 & 0.5 & 4.46 & 0.39 & 4.0 & 4.9 & Sill 1190.4: & 2.4255 \\
\hline 8 & 4081.0 & 0.5 & 2.25 & 0.26 & 3.5 & 3.9 & SiII 1190.4: & 2.4283 \\
\hline 9 & 4090.3 & 0.5 & 5.25 & 0.37 & 5.5 & 5.1 & SiII 1193.3 & 2.4277 \\
\hline 10 & 4107.4 & 0.5 & 6.06 & 0.29 & 1.5 & 6.5 & $\ldots \ldots \ldots . .$. & $\ldots \ldots$ \\
\hline 11 & 4113.8 & 0.5 & 3.32 & 0.21 & 4.5 & 6.8 & .............. & ...... \\
\hline 12 & 4119.6 & 0.5 & 2.57 & 0.24 & 6.0 & 6.8 & .................. & ....... \\
\hline 13 & 4134.7 & 0.5 & 4.12 & 0.24 & 5.0 & 7.0 & SiIII 1206.5 & 2.4270 \\
\hline 14 & 4139.6 & 0.5 & 1.45 & 0.22 & 3.0 & 5.6 & FeII 1144.9 & 2.6157 \\
\hline 15 & 4221.0 & 0.5 & 1.62 & 0.28 & 3.0 & 4.7 & .................. & ....... \\
\hline 16 & 4233.0 & 0.5 & 4.69 & 0.37 & 6.5 & 5.4 & ............ & ....... \\
\hline 17 & 4245.8 & 0.5 & 1.29 & 0.26 & 3.5 & 4.8 & ............. & ...... \\
\hline 18 & 4259.0 & 0.5 & 4.03 & 0.36 & 4.5 & 5.4 & ................. & ...... \\
\hline 19 & 4304.2 & 0.5 & 1.31 & 0.22 & 2.5 & 5.1 & SiII 1190.4 & 2.6158 \\
\hline 20 & 4314.5 & 0.5 & 2.64 & 0.26 & 3.5 & 5.2 & SiII 1193.3 & 2.6156 \\
\hline 21 & 4319.7 & 0.5 & 4.44 & 0.35 & 6.5 & 5.0 & SiII 1260.4 & 2.4272 \\
\hline 22 & 4326.0 & 0.5 & 3.62 & 0.35 & 5.0 & 5.0 & ................ & ....... \\
\hline 23 & 4347.5 & 0.5 & 1.86 & 0.30 & 2.5 & 3.5 & ............ & ....... \\
\hline 24 & 4356.1 & 0.5 & 4.64 & 0.49 & 5.0 & 3.1 & …........ & ...... \\
\hline 25 & 4462.5 & 0.5 & 5.72 & 0.53 & 9.5 & 4.0 & OI 1302.2 & 2.4269 \\
\hline 26 & 4471.5 & 0.5 & 6.46 & 0.58 & 7.5 & 4.1 & SilI 1304.4 & 2.4280 \\
\hline 27 & 4509.5 & 0.5 & 2.88 & 0.38 & 3.0 & 4.2 & ................. & ...... \\
\hline 28 & 4536.3 & 0.5 & 3.90 & 0.41 & 6.0 & 4.4 & ............. & $\ldots \ldots$ \\
\hline 29 & 4546.7 & 0.5 & 3.54 & 0.41 & 3.0 & 4.4 & ……....... & ...... \\
\hline 30 & 4559.9 & 0.6 & 10.65 & 0.58 & 15.0 & 4.8 & SiII 1260.4 & 2.6178 \\
\hline 31 & 4574.0 & 0.5 & 8.49 & 0.52 & 9.5 & 4.8 & CII 1334.5 & 2.4275 \\
\hline 32 & 4583.0 & 0.5 & 1.49 & 0.29 & 4.5 & 4.2 & ............. & ....... \\
\hline 33 & 4587.0 & 0.5 & 1.13 & 0.28 & 3.0 & 4.1 & ............ & ...... \\
\hline 34 & 4592.3 & 0.5 & 2.71 & 0.36 & 4.0 & 4.4 & ............. & ...... \\
\hline 35 & 4599.5 & 0.5 & 2.75 & 0.40 & 4.0 & 4.5 & ............ & ....... \\
\hline 36 & 4621.8 & 0.7 & 2.15 & 0.47 & 9.5 & 4.5 & …......... & ....... \\
\hline 37 & 4641.3 & 0.5 & 3.69 & 0.39 & 5.0 & 4.7 & ........... & ....... \\
\hline 38 & 4646.3 & 0.5 & 2.90 & 0.38 & 2.0 & 4.6 & ........... & ....... \\
\hline 39 & 4658.9 & 0.5 & 2.58 & 0.38 & 5.0 & 4.6 & ............ & ...... \\
\hline 40 & 4670.1 & 0.5 & 1.91 & 0.31 & 2.0 & 4.3 & ............ & ....... \\
\hline 41 & 4678.5 & 0.6 & 2.03 & 0.35 & 5.5 & 4.3 & ............ & ...... \\
\hline 42 & 4684.7 & 0.5 & 1.10 & 0.25 & 1.5 & 4.1 & ........... & ....... \\
\hline 43 & 4690.0 & 0.5 & 3.30 & 0.42 & 3.0 & 4.6 & ............... & ....... \\
\hline 44 & 4708.0 & 0.5 & 3.48 & 0.38 & 6.0 & 4.7 & OI 1302.2 & 2.6154 \\
\hline 45 & 4743.8 & 0.5 & 4.12 & 0.44 & 3.5 & 5.0 & ............. & ....... \\
\hline 46 & 4756.5 & 0.5 & 1.88 & 0.32 & 3.5 & 4.7 & ............ & ....... \\
\hline 47 & 4778.8 & 0.5 & 7.96 & 0.49 & 9.0 & 5.7 & SiIV 1393.8: & 2.4286 \\
\hline 48 & 4790.4 & 0.5 & 2.11 & 0.34 & 2.5 & 5.8 & ............ & $\ldots \ldots$ \\
\hline 49 & 4819.1 & 0.5 & 1.60 & 0.21 & 2.5 & 6.4 & .............. & ....... \\
\hline 50 & 4826.1 & 0.5 & 4.38 & 0.26 & 4.0 & 7.6 & CII 1334.5 & 2.6164 \\
\hline 51 & 4831.5 & 0.5 & 0.90 & 0.17 & 2.5 & 6.8 & CII $^{*}$ 1335.7: & 2.6172 \\
\hline 52 & 4837.9 & 0.5 & 2.72 & 0.22 & 6.0 & 8.0 & ............. & ....... \\
\hline 53 & 4849.9 & 0.5 & 1.28 & 0.16 & 2.5 & 8.3 & ............ & $\ldots \ldots$ \\
\hline 54 & 4872.2 & 0.5 & 2.04 & 0.13 & 3.5 & 11.2 & ............ & $\ldots \ldots$ \\
\hline 55 & 4884.2 & 0.5 & 0.94 & 0.15 & 6.0 & 11.9 & ............ & $\ldots \ldots$ \\
\hline 56 & 4918.3 & 0.5 & 0.85 & 0.16 & 4.0 & 9.1 & ............. & ...... \\
\hline 57 & 4954.4 & 0.5 & 1.90 & 0.17 & 3.5 & 8.2 & CIV 1548.2 & 2.2001 \\
\hline 58 & 4958.2 & 0.5 & 1.12 & 0.14 & 3.0 & 7.9 & CIV 1548.2 & 2.2026 \\
\hline 59 & 4964.1 & 0.5 & 2.24 & 0.20 & 3.0 & 8.1 & CIV 1550.8 & 2.2010 \\
\hline 60 & 4970.3 & 0.5 & 0.91 & 0.19 & 3.0 & 7.4 & ............ & $\ldots .$. \\
\hline 61 & 4975.5 & 0.5 & 0.91 & 0.18 & 2.5 & 7.0 & ............ & $\ldots \ldots$ \\
\hline 62 & 5211.5 & 0.5 & 2.47 & 0.45 & 3.5 & 3.3 & ............... & ....... \\
\hline 63 & 5231.4 & 0.6 & 1.90 & 0.45 & 3.5 & 3.4 & SilI 1526.7 & 2.4266 \\
\hline 64 & 5306.1 & 0.5 & 2.39 & 0.43 & 5.0 & 3.5 & CIV 1548.2 & 2.4273 \\
\hline
\end{tabular}




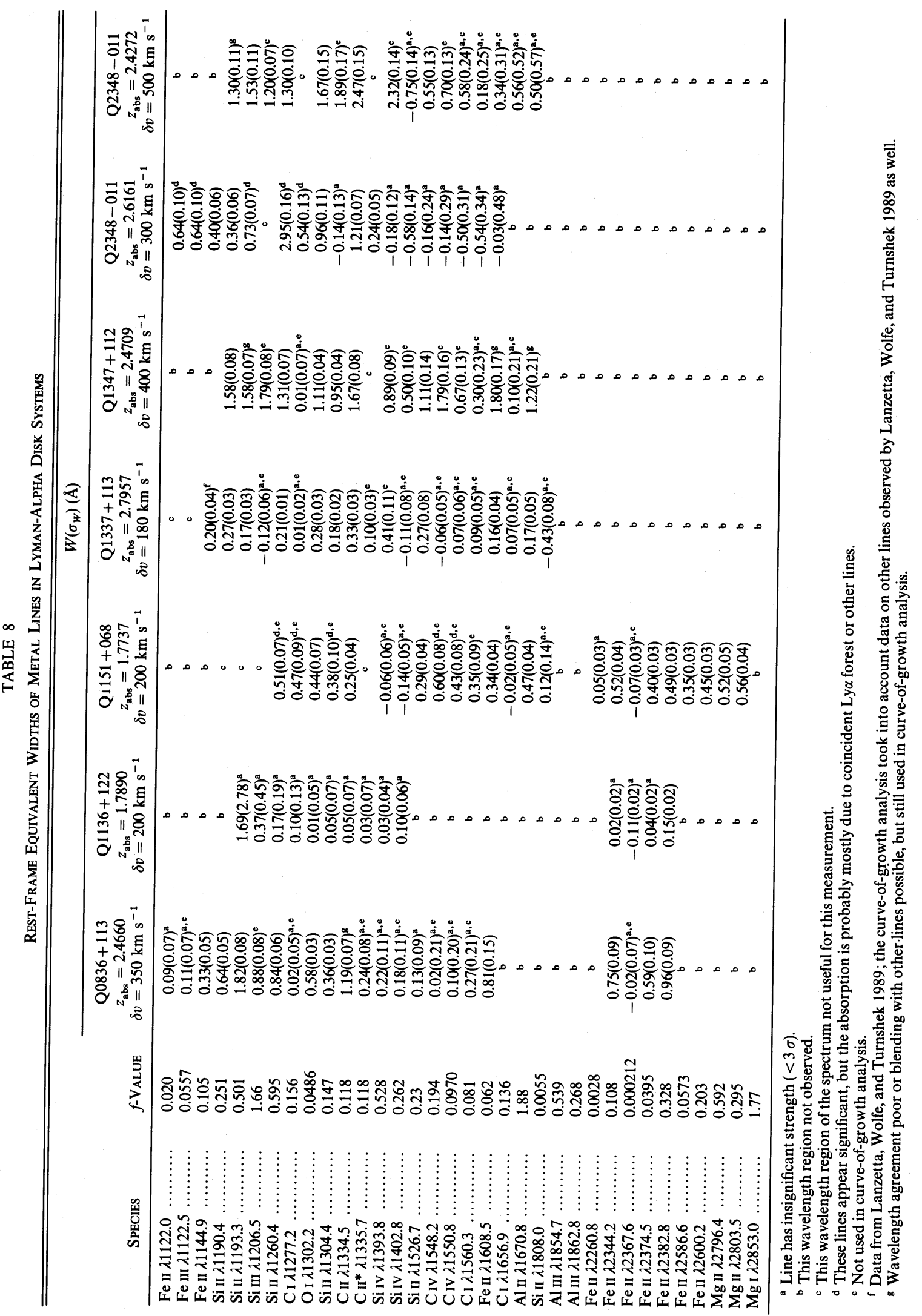


TABLE 9

Properties of Damped Lyman-Alpha Systems Studied Here

\begin{tabular}{|c|c|c|c|c|c|}
\hline QSO & $z_{\text {abs }}$ & $\begin{array}{c}\Delta v_{\text {metals }} \\
\left(\mathrm{km} \mathrm{s}^{-1}\right)\end{array}$ & $\underset{\left(\mathrm{km} \mathrm{s}^{-1}\right)}{\Delta v_{\mathrm{H}^{\prime}}}$ & 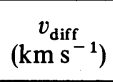 & $\log N(\mathrm{H} \mathrm{I})$ \\
\hline $\mathrm{Q} 0836+113$. & 2.4660 & $\leq 150$ & 2500 & $<50$ & 20.60 \\
\hline $\mathrm{Q} 1136+122$. & 1.7890 & $<110$ & 1200 & $<60$ & 20.00 \\
\hline Q1151+068.. & 1.7737 & $<110$ & 5200: & $<800$ & 21.26: \\
\hline $\mathrm{Q} 1337+113 \ldots$ & 2.7957 & $<120$ & 3500 & $<50$ & 20.90 \\
\hline $\mathrm{Q} 1347+112 \ldots$ & 2.4709 & 400 & 1800 & $<50$ & 20.30 \\
\hline 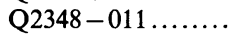 & 2.6161 & $\leq 200$ & 5200 & $<100$ & 21.26 \\
\hline Q2348-011 $\ldots$ & 2.4272 & 500 & 2100 & $<180$ & 20.48 \\
\hline
\end{tabular}

TABLE 10

EFFECTIVE VeLOCITY DISPERSIONS DETERMINED FROM CURVE-OF-Growth ANALYSIS

\begin{tabular}{|c|c|c|c|c|}
\hline QSO & $z_{\mathrm{abs}}$ & $\left(\begin{array}{c}\sigma \\
\left(\mathrm{km} \mathrm{s}^{-1}\right)\end{array}\right.$ & $\begin{array}{c}+\sigma_{\sigma} \\
\left(\mathrm{km} \mathrm{s}^{-1}\right)\end{array}$ & $\left(\begin{array}{c}-\sigma_{\sigma} \\
\left(\mathrm{km} \mathrm{s}^{-1}\right)\end{array}\right.$ \\
\hline $\mathrm{Q} 0836+113 .$. & 2.4660 & 50 & 6.1 & 5.4 \\
\hline $\mathrm{Q} 1136+122 \ldots$ & 1.7890 & & & \\
\hline Q1151+068 $\ldots$ & 1.7737 & 8.5 & 0.9 & 1.1 \\
\hline $\mathrm{Q} 1337+113 \ldots \ldots \ldots \ldots$ & 2.7957 & 8.3 & 0.4 & 1.1 \\
\hline $\mathrm{Q} 1347+112 \ldots \ldots \ldots \ldots$ & 2.4709 & 71 & 4.5 & 4.5 \\
\hline Q2348-011 $\ldots \ldots \ldots \ldots$ & 2.6161 & & & \\
\hline $\mathrm{Q} 2348-011 \ldots \ldots \ldots \ldots$ & 2.4272 & 71 & 4.2 & 11.0 \\
\hline
\end{tabular}

TABLE 11

Metal Line Column Densities from the Curve-of-Growth Analysis of $z_{\text {abs }}=2.4660 \mathrm{IN} \mathrm{Q0836+113}$

\begin{tabular}{cccccc}
\hline \hline Ion & $\begin{array}{c}\log N \\
\left(\mathrm{~cm}^{-2}\right)\end{array}$ & $\begin{array}{c}+\sigma_{\log N} \\
\left(\mathrm{~cm}^{-2}\right)\end{array}$ & $\begin{array}{c}-\sigma_{\log } N \\
\left(\mathrm{~cm}^{-2}\right)\end{array}$ & $\begin{array}{c}\log N_{\min } \\
\left(\mathrm{cm}^{-2}\right)\end{array}$ & $\begin{array}{c}\log N_{\max } \\
\left(\mathrm{cm}^{-2}\right)\end{array}$ \\
\hline $\mathrm{C}^{+} \ldots \ldots \ldots \ldots$ & 15.8 & 0.4 & 0.3 & 14.8 & 19.2 \\
$\mathrm{O}^{0} \ldots \ldots \ldots \ldots .1$ & 15.1 & 0.1 & 0.1 & 14.9 & 18.7 \\
$\mathrm{Si}^{+} \ldots \ldots \ldots \ldots$ & 14.5 & 0.1 & 0.1 & 14.2 & 17.6 \\
$\mathrm{Fe}^{+} \ldots \ldots \ldots$. & 14.3 & 0.1 & 0.1 & 14.4 & 17.4 \\
\hline
\end{tabular}

TABLE 12

Metal Line Column Densities from the Curve-OF-Growth Analysis of $z_{\text {abs }}=1.7737$ IN Q1151+068

\begin{tabular}{cccccc}
\hline \hline Ion & $\begin{array}{c}\log N \\
\left(\mathrm{~cm}^{-2}\right)\end{array}$ & $\begin{array}{c}+\sigma_{\log N} \\
\left(\mathrm{~cm}^{-2}\right)\end{array}$ & $\begin{array}{c}-\sigma_{\log N} \\
\left(\mathrm{~cm}^{-2}\right)\end{array}$ & $\begin{array}{c}\log N_{\min } \\
\left(\mathrm{cm}^{-2}\right)\end{array}$ & $\begin{array}{c}\log N_{\max } \\
\left(\mathrm{cm}^{-2}\right)\end{array}$ \\
\hline $\mathrm{C}^{+} \ldots \ldots \ldots \ldots$ & 15.7 & 0.9 & 0.7 & 14.1 & 17.8 \\
$\mathrm{O}^{0} \ldots \ldots \ldots \ldots$ & 18.2 & 0.2 & 0.4 & 14.8 & 18.4 \\
$\mathrm{Al}^{+} \ldots \ldots \ldots \ldots$ & 15.7 & 0.2 & 0.3 & 13.0 & 16.0 \\
$\mathrm{Mg}^{+} \ldots \ldots \ldots$. & 15.2 & 0.6 & 0.5 & 13.4 & 16.5 \\
$\mathrm{Si}^{+} \ldots \ldots \ldots \ldots$ & 15.5 & 0.7 & 0.7 & 13.5 & 17.0 \\
$\mathrm{Fe}^{+} \ldots \ldots \ldots \ldots$ & 15.4 & 0.5 & 0.3 & 14.4 & 16.8 \\
\hline
\end{tabular}

TABLE 13

Metal Line Column Densities from the Curve-OF-Growth Analysis of $z_{\text {abs }}=2.7957$ IN Q1337+113

\begin{tabular}{cccccc}
\hline \hline Ion & $\begin{array}{c}\log N \\
\left(\mathrm{~cm}^{-2}\right)\end{array}$ & $\begin{array}{c}+\sigma_{\log N} \\
\left(\mathrm{~cm}^{-2}\right)\end{array}$ & $\begin{array}{c}-\sigma_{\log N} \\
\left(\mathrm{~cm}^{-2}\right)\end{array}$ & $\begin{array}{c}\log N_{\min } \\
\left(\mathrm{cm}^{-2}\right)\end{array}$ & $\begin{array}{c}\log N_{\max } \\
\left(\mathrm{cm}^{-2}\right)\end{array}$ \\
\hline $\mathrm{C}^{+} \ldots \ldots \ldots \ldots$ & 17.3 & 0.4 & 0.6 & 14.3 & 18.1 \\
$\mathrm{O}^{0} \ldots \ldots \ldots \ldots$ & 17.0 & 0.5 & 0.6 & 14.6 & 18.0 \\
$\mathrm{Al}^{+} \ldots \ldots \ldots \ldots$ & 13.0 & 0.6 & 0.4 & 12.6 & 15.2 \\
$\mathrm{Si}^{+} \ldots \ldots \ldots \ldots$. & 14.7 & 0.5 & 0.3 & 13.9 & 15.5 \\
$\mathrm{Fe}^{+} \ldots \ldots \ldots \ldots$ & 14.7 & 0.2 & 0.2 & 14.2 & 17.5 \\
\hline
\end{tabular}

TABLE 14

Metal Line Column Densities from the Curve-of-Growth Analysis of $z_{\text {abs }}=2.4709 \mathrm{IN} \mathrm{Q} 1347+112$

\begin{tabular}{cccccc}
\hline \hline Ion & $\begin{array}{c}\log N \\
\left(\mathrm{~cm}^{-2}\right)\end{array}$ & $\begin{array}{c}+\sigma_{\log N} \\
\left(\mathrm{~cm}^{-2}\right)\end{array}$ & $\begin{array}{c}-\sigma_{\log N} \\
\left(\mathrm{~cm}^{-2}\right)\end{array}$ & $\begin{array}{c}\log N_{\min } \\
\left(\mathrm{cm}^{-2}\right)\end{array}$ & $\begin{array}{c}\log N_{\text {max }} \\
\left(\mathrm{cm}^{-2}\right)\end{array}$ \\
\hline $\mathrm{C}^{+} \ldots \ldots \ldots \ldots$ & 15.9 & 0.2 & 0.2 & 15.0 & 19.5 \\
$\mathrm{O}^{0} \ldots \ldots \ldots \ldots$. & 15.6 & 0.1 & 0.1 & 15.2 & 19.2 \\
$\mathrm{Al}^{+} \ldots \ldots \ldots \ldots$ & 13.7 & 0.2 & 0.2 & 13.4 & 16.9 \\
$\mathrm{Si}^{+} \ldots \ldots \ldots \ldots$ & 15.0 & 0.1 & 0.1 & 14.6 & 17.5 \\
$\mathrm{Fe}^{+} \ldots \ldots \ldots$. & 15.8 & 0.3 & 0.2 & 15.1 & 19.6 \\
\hline
\end{tabular}

TABLE 15

Metal Line Column Densities from the Curve-of-Growth ANalysis of $z_{\text {abs }}=2.4272$ IN Q2348-011

\begin{tabular}{cccccc}
\hline \hline Ion & $\begin{array}{c}\log N \\
\left(\mathrm{~cm}^{-2}\right)\end{array}$ & $\begin{array}{c}+\sigma_{\log N} \\
\left(\mathrm{~cm}^{-2}\right)\end{array}$ & $\begin{array}{c}-\sigma_{\log } N \\
\left(\mathrm{~cm}^{-2}\right)\end{array}$ & $\begin{array}{c}\log N_{\min } \\
\left(\mathrm{cm}^{-2}\right)\end{array}$ & $\begin{array}{c}\log N_{\max } \\
\left(\mathrm{cm}^{-2}\right)\end{array}$ \\
\hline $\mathrm{C}^{+} \ldots \ldots \ldots \ldots$ & 17.7 & 1.1 & 0.8 & 14.8 & 19.2 \\
$\mathrm{O}^{0} \ldots \ldots \ldots \ldots$. & 16.4 & 0.6 & 0.4 & 15.1 & 19.1 \\
$\mathrm{Si}^{+} \ldots \ldots \ldots \ldots$ & 14.8 & 0.2 & 0.1 & 14.1 & 16.8 \\
\hline
\end{tabular}

$\Delta v_{\mathrm{HI}} \simeq 1800 \mathrm{~km} \mathrm{~s}^{-1}$. A curve-of-growth analysis, illustrated in Figure 12 and based on five $\mathrm{Si}$ II lines, indicates that $\sigma \simeq 71$ $\mathrm{km} \mathrm{s}^{-1}$.

$$
\text { vi) The } Q 2348+011 z_{\text {abs }}=2.6161 \text { System }
$$

The strong Ly $\alpha$ absorption feature near $4400 \AA$ is moderately well fitted by a damping profile with $N(\mathrm{H} \mathrm{I}) \simeq 1.8 \times 10^{21}$ $\mathrm{cm}^{-2}$. The quality of the fit is degraded by the presence of some sharp, narrow absorption on the shortward side of the profile. This appears to be a system with strong low-ion absorption and weak high-ion absorption, although the possibility of $\mathrm{C}$ II being blended with other absorption lines is hard to evaluate. The values $v_{\text {diff }}<100 \mathrm{~km} \mathrm{~s}^{-1}$ and $\Delta v_{\text {metals }} \leq 200$ $\mathrm{km} \mathrm{s}^{-1}$, while $\Delta v_{\mathrm{HI}} \simeq 5200 \mathrm{~km} \mathrm{~s}^{-1}$, support the conclusion that the feature is damped. Three $\mathrm{Si}$ II lines appear to be definitely present, but both the Si II $\lambda 1193$ and $\mathrm{Si}$ II $\lambda 1260$ identification are confused, and blending with other absorption lines is likely. Because of this and the lack of red-wavelength data, no curve-of-growth analysis was performed. Significant excess flux may be present near the center of the profile; if this is real and the Ly $\alpha$ feature is indeed damped, this could be attributed to emission from the high-redshift disk galaxy causing the absorption.

$$
\text { vii) The } Q 2348-011 z_{\text {abs }}=2.4272 \text { System }
$$

This is a second strong Ly $\alpha$ absorption system in the spectrum of Q2348 - 011, displaced $\sim 16,000 \mathrm{~km} \mathrm{~s}^{-1}$ shortward of the higher redshift system. The absorption profile is moderately well fitted by a damping profile with $N(\mathrm{H} \mathrm{I}) \simeq 3 \times 10^{20}$ $\mathrm{cm}^{-2}$. Although the signal-to-noise ratio in this part of the spectrum is only $\sim 6$ per resolution element, the quality of the fit improved after a weak Ly $\beta$ emission feature was assumed to be present at $4120 \AA$ and fitted with a narrow Gaussian function. Low-ion absorption is clearly present, but $\mathrm{C}$ IV absorption appears weak, with only the C IV $\lambda 1548$ line possibly present. Damping is also indicated, since $v_{\text {diff }}<180 \mathrm{~km} \mathrm{~s}^{-1}$ and $\Delta v_{\text {metals }} \simeq 500 \mathrm{~km} \mathrm{~s}^{-1}$, while $\Delta v_{\mathrm{H} \mathrm{I}} \simeq 2100 \mathrm{~km} \mathrm{~s}^{-1}$. A curve-of-growth analysis, illustrated in Figure 13 and based on four Si II lines, indicates that $\sigma \simeq 71 \mathrm{~km} \mathrm{~s}^{-1}$. Note that the wavelength of the Si II $\lambda 1190$ line is somewhat discrepant, but it was included in the curve-of-growth analysis. However, the 


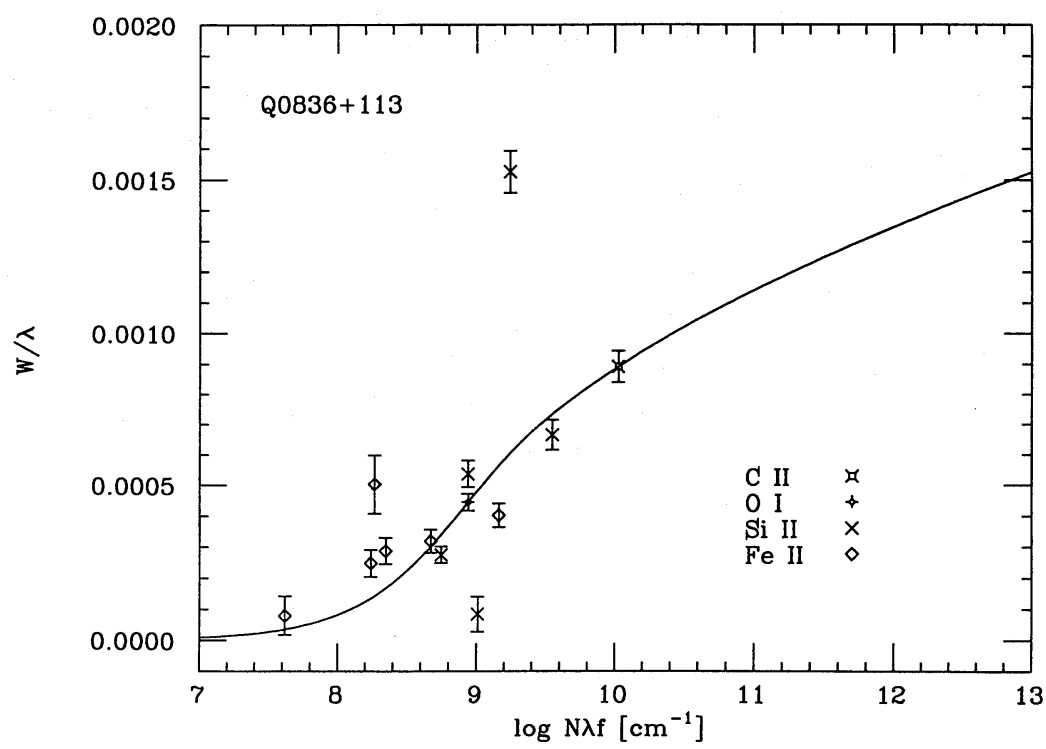

Fig. 8. - Curve of growth for the Q0836+113 $z_{\text {abs }}=2.4660$ system

Si II $\lambda 1304$ rest equivalent width was probably too high as a result of blending, as indicated by a slight blue asymmetry on the absorption profile, and it was not included in the curve-ofgrowth analysis.

\section{c) Components on Other Damped Lyman-Alpha Systems from the Lick Survey}

Besides the seven damped Ly $\alpha$ systems discussed above, strong evidence supports the identification of 11 additional damped $\mathrm{Ly} \alpha$ systems selected from the Lick low-resolution spectroscopic survey. In each case the identification is based on the fit of a Voigt damping profile to the Ly $\alpha$ feature and on the coincidence of low-ion metal lines at the Ly $\alpha$ redshift, i.e., on criteria 1 and 2 discussed in $\S$ III $a$. In some cases the identification is strengthened by the detection of higher order Lyman lines (i.e., criterion 3). The 11 additional systems are discussed extensively in Paper IV and in publications by other groups. Rather than repeat the evidence here, we summarize the essential properties of the 11 systems in Table 16. In $\S$ IVd abundance determinations which have been made for some of these systems are discussed.

\section{DISCUSSION}

The seven systems that have been studied are representative of the damped Ly $\alpha$ population of QSO absorbers. The information presented on them included (1) heliocentric vacuum redshifts accurate to a few times $10^{-4},(2)$ neutral hydrogen column densities typically accurate to better than a factor of $\sim 50 \%$, (3) equivalent widths for the corresponding metal lines and associated statistical errors (systematic errors were not considered), (4) the velocity extent of the metal line absorption, and (5) results from a standard curve-of-growth analysis of the

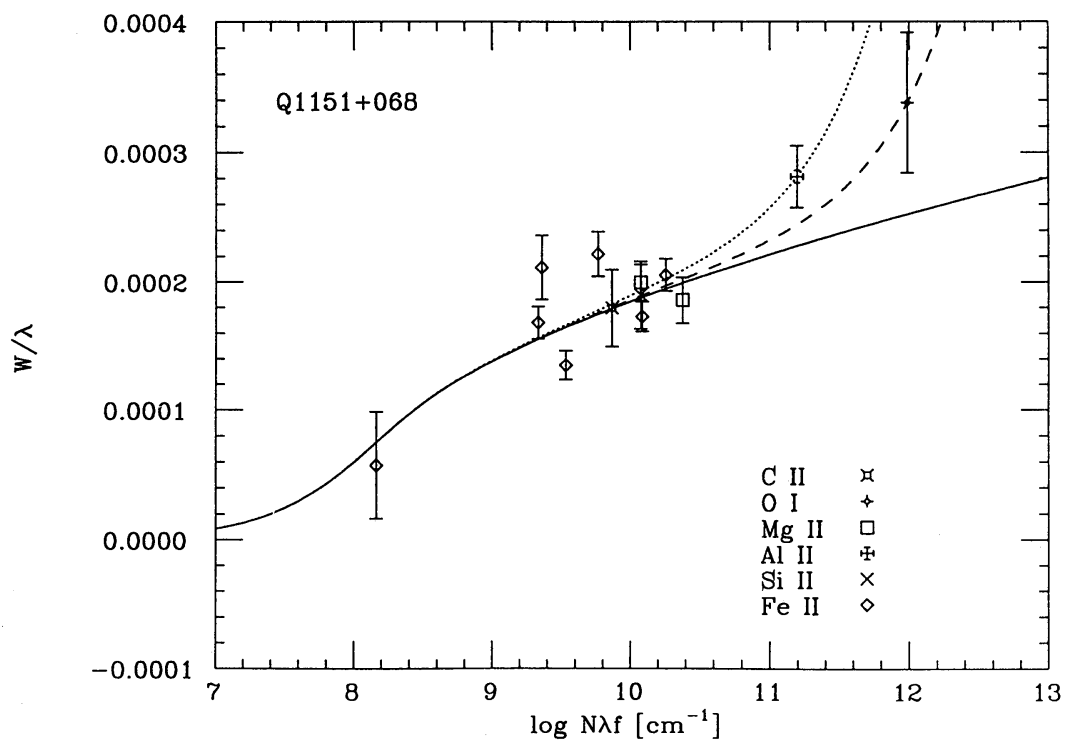

Fig. 9.-Curve of growth for the Q1151+068 $z_{\text {abs }}=1.7737$ system. The curve plotted with long dashes represents the curve of growth for $\mathrm{O} \mathrm{I}$, while the curve plotted with dots represents the curve of growth for Al II. 


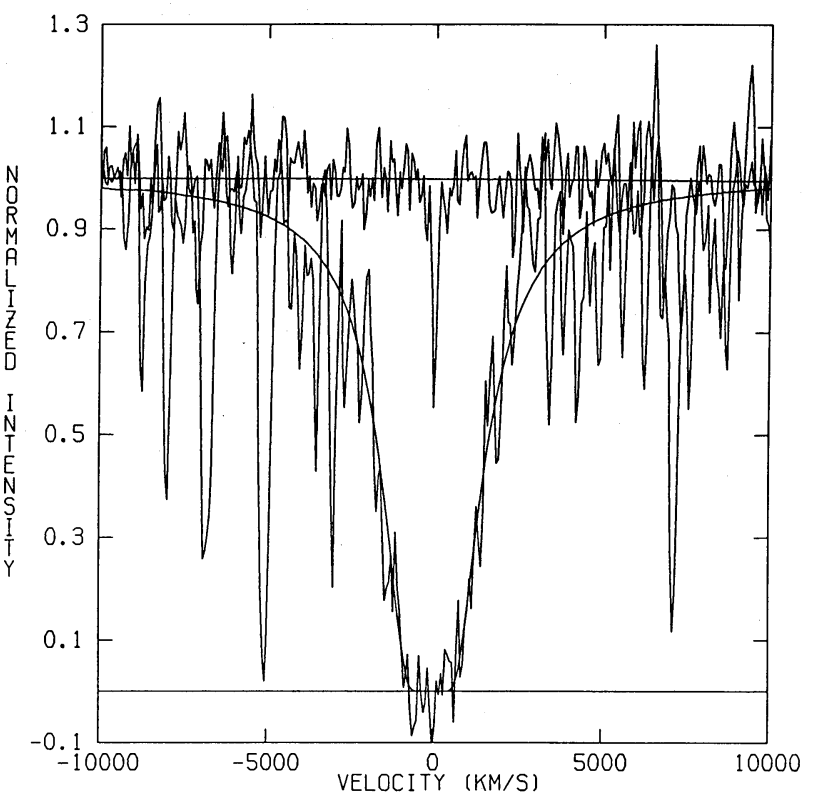

FIG. 10.-Velocity profile of the $C$ II line in the Q1337+113 $z_{\text {abs }}=2.7957$ system superposed on the inferred velocity profile of the $\mathrm{H} \mathrm{I}$ at the same redshift. low-ion metal lines in five of the seven systems. We showed that damping could be deduced from the quality of the fit of a Voigt damping profile to the Ly $\alpha$ data, the comparison of the metal line and inferred Ly $\alpha$ velocity profiles, and the consistency of the profiles of higher order Lyman lines with damped Ly $\alpha$ profiles. A summary of the observed properties of these systems is now given, and our interpretation of them is discussed. The differences between these systems and other types of QSO narrow metal line systems and abundance determinations are also discussed. Finally, a status report on the incidence of damped $\operatorname{Ly} \alpha$ absorption in the Lick survey is presented.

\section{a) Properties of the Metal Lines of Damped Lyman-Alpha Systems}

The seven systems studied lie in the redshift range $1.77<$ $z_{\text {abs }}<2.80$ and have $\mathrm{H}$ I column densities $N(\mathrm{H} \mathrm{I})=1 \times 10^{20}$ to $1.8 \times 10^{21} \mathrm{~cm}^{-2}$. The $C$ IV region was observed in six of the seven systems. In four cases no significant $C$ IV absorption with rest-frame equivalent width $W>0.3-0.6 \AA$ was detected, and in the remaining two cases the detected $\mathrm{C}$ IV absorption was comparable to or much weaker than the corresponding C II absorption. The dominance of low ions over high ions is illustrated in Figure 14, where $W(\mathrm{Mg}$ II $)$ versus $W(\mathrm{C}$ IV) is plotted

TABLE 16

Properties of Other Damped Lyman-Alpha Systems Identified in the Lick SURVey

\begin{tabular}{|c|c|c|c|}
\hline QSO & $z_{\text {abs }}$ & $\log N\left(\mathrm{H} \mathrm{I}_{\mathrm{I}}\right)$ & Reference \\
\hline $\mathrm{Q} 0100+103 \ldots$ & 2.309 & 21.40 & Black, Chafee, and Foltz 1987 \\
\hline $\mathrm{Q} 0149+336$ & 2.140 & 20.60 & Paper IV \\
\hline Q0458-020 & 2.039 & 21.70 & Wolfe $e t$ al. and Paper IV \\
\hline $\mathrm{Q} 1215+333 \ldots \ldots \ldots$ & 2.001 & 21.00 & Paper IV \\
\hline $\mathrm{Q} 1244+347 \ldots \ldots \ldots \ldots$ & 1.860 & 20.60 & Paper IV \\
\hline $\mathrm{Q} 1337+113 \ldots \ldots \ldots \ldots$ & 2.507 & 20.00 & Lanzetta, Wolfe, and Turnshek 1989 \\
\hline $\mathrm{Q} 2136+141 \ldots \ldots \ldots$ & 2.138 & 20.30 & Paper IV \\
\hline Q2206-199N ........ & 1.920 & 20.85 & Robertson, Shaver, and Carswell 1983 \\
\hline Q2206-199N ........ & 2.077 & 20.70 & Robertson, Shaver, and Carswell 1983 \\
\hline $\mathrm{Q} 2359-022 \ldots \ldots \ldots$ & 2.095 & 20.70 & Paper IV \\
\hline $\mathrm{Q} 2359-022$ & 2.154 & 20.30 & Paper IV \\
\hline
\end{tabular}

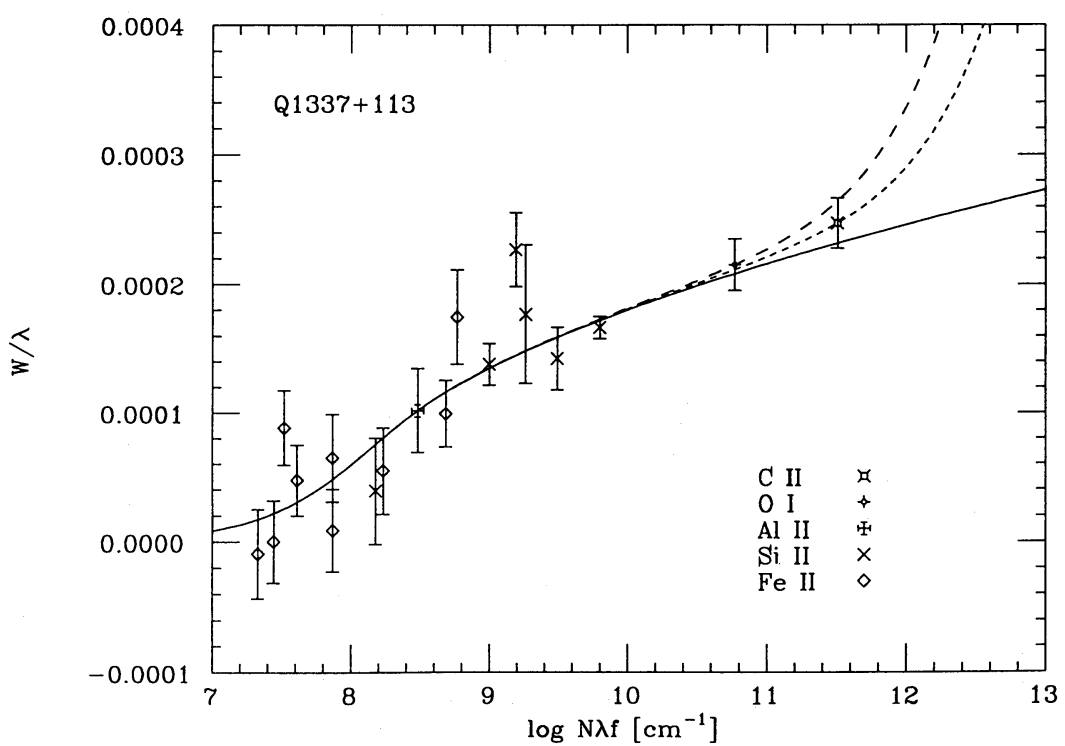

FIG. 11.-Curve of growth for the Q1337+113 $z_{\text {abs }}=2.7957$ system. The curve plotted with long dashes represents the curve of growth for O I, while the curve plotted with short dashes represents the curve of growth for $\mathrm{C}$ II. 


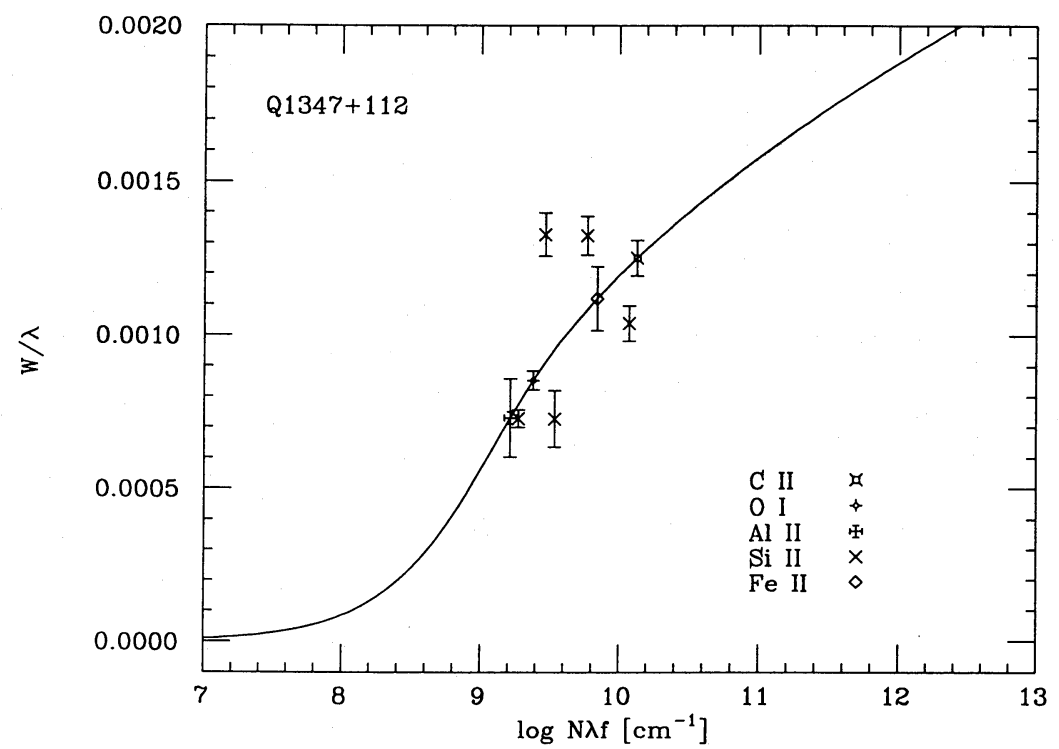

Fig. 12.-Curve of growth for the Q1347+112 $z_{\text {abs }}=2.4709$ system

for the six systems for which the $\mathrm{C}$ IV regions were observed. If the $\mathrm{Mg}$ II region was not observed, $W(\mathrm{Mg}$ II $)>W(\mathrm{C}$ II) is assumed and an inferred lower limit on $W(\mathbf{M g}$ II) is plotted in Figure 14 (the data of LTW indicate that this is valid).

The velocity extent of the metal line absorption, $\Delta v_{\text {metals }}$, was determined by directly measuring the wavelength range over which absorption was present. In two cases the absorption was clearly resolved, with $\Delta v_{\text {metals }} \simeq 400-500 \mathrm{~km} \mathrm{~s}^{-1}$. In two other cases the absorption was possibly resolved, with $\Delta v_{\text {metals }} \leq$ 150-200 $\mathrm{km} \mathrm{s}^{-1}$. In the remaining three cases the absorption was clearly unresolved, with $\Delta v_{\text {metals }}<120 \mathrm{~km} \mathrm{~s}^{-1}$. The effective velocity dispersion, $\sigma$, of the low-ion gas was investigated in five of the seven cases by performing a curve-of-growth analysis. The formal errors in $\sigma$, which were presented in Table 10 , are likely to be substantially smaller than the true errors; however, we believe that the true errors in $\sigma$ are small enough to distinguish a quiescent component from a turbulent component. In three of the five systems the effective velocity disper- sion of the low ions was found to lie in the range $\sigma \simeq 20-71 \mathrm{~km}$ $\mathrm{s}^{-1}$. Since two of these three systems have resolved absorption with $\Delta v_{\text {metals }} \simeq 400-500 \mathrm{~km} \mathrm{~s}^{-1}$ and since the other system probably has resolved absorption with $\Delta v_{\text {metals }} \simeq 150 \mathrm{~km} \mathrm{~s}^{-1}$, the result that $\Delta v_{\text {metals }}>\sigma$ indicates that in these three systems the observed metal lines arise from turbulent gas consisting of several components. The remaining two cases, the $z_{\text {abs }}=$ 1.7737 system toward Q1151+068 and the $z_{\text {abs }}=2.7957$ system toward Q1337+113, were found to have unresolved metal line absorption and a low effective velocity dispersion, $\sigma<10 \mathrm{~km} \mathrm{~s}^{-1}$, indicating that only quiescent components were detected. The best-fit column densities for the low ions in the systems were also inferred (Tables 11-15); however, because of the moderate resolution of the data, these column densities should be considered strictly lower limits. The constraints which they may impose on relative and absolute abundances are reviewed in $\S \mathrm{IV} c$, after additional discussion and interpretation are presented in $\S \mathrm{IV} b$.

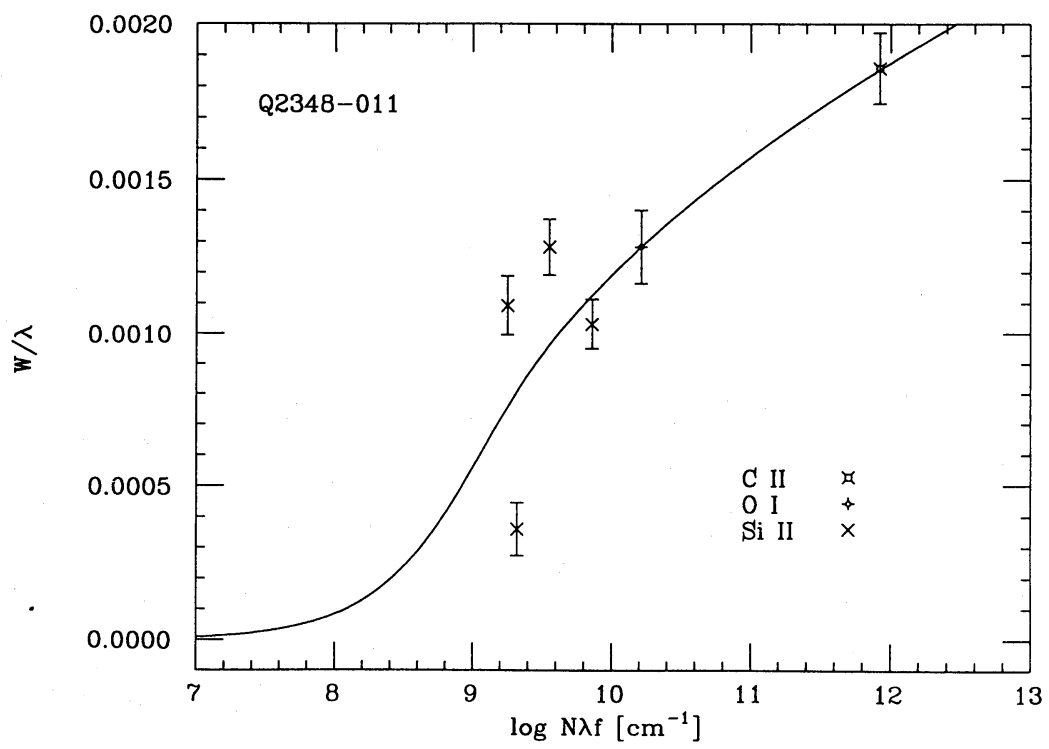

Fig. 13.-Curve of growth for the Q2348-011 $z_{\text {abs }}=2.4272$ system 


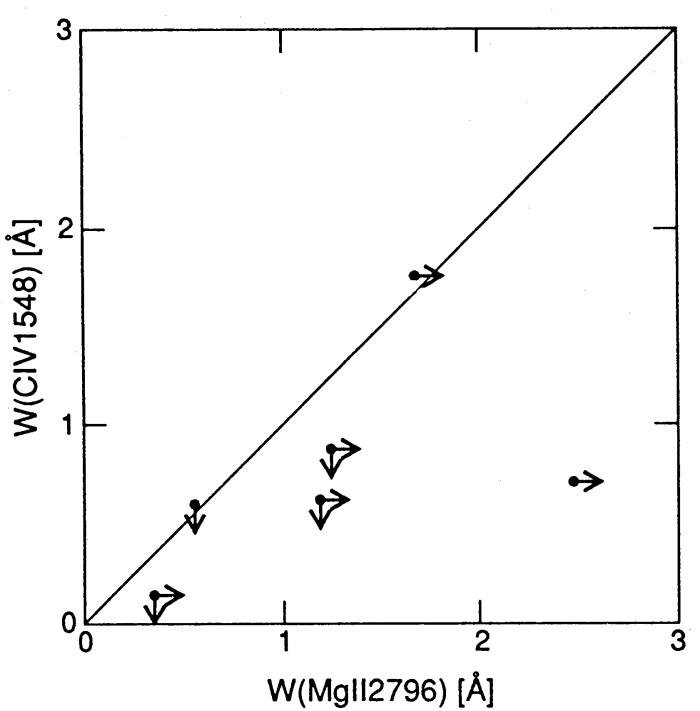

Fig. 14.-Plot of $W(\mathrm{Mg}$ II) vs. $W(\mathrm{C}$ Iv) for six damped Ly $\alpha$ systems in which the $\mathrm{C}$ IV wavelength region was observed. When the $\mathrm{Mg}$ II wavelength region was not accessible, $W(\mathrm{Mg}$ II $)>W(\mathrm{C}$ II) was assumed, so that a lower limit for $W(\mathrm{Mg}$ II) could be plotted. When $\mathrm{C}$ IV was not detected, the $3 \sigma$ upper limit on $W(\mathrm{C}$ IV $)$ was plotted.

\section{b) Comparisons between Damped Lyman-Alpha Systems and Other Narrow Metal Absorption Line Systems}

A comparison of the properties of the damped Ly $\alpha$ systems, selected on the basis of identifying damped Ly $\alpha$ in QSO absorption-line spectra, with the properties of other QSO absorption-line systems is worthwhile. Such comparisons, when coupled with theoretical work, will eventually help clarify how the various types of QSO absorbers are interrelated, and will ultimately allow us to determine which absorption systems are due to galactic halos and disks. A comparison of properties is particularly valuable since the metal line properties of the damped Ly $\alpha$ systems are unbiased, because essentially any absorber with a total column density in excess of $\sim 2 \times 10^{20} \mathrm{~cm}^{-2}$ must be selected by our survey technique. The characteristics which differentiate the damped Ly $\alpha$ systems from other narrow metal line systems are discussed below.

We discuss the damped systems in the context of three "types" of narrow metal line systems: (1) "optically thick systems," which are not correlated with a QSO's emission redshift; (2) "optically thin systems," which are not correlated with a QSO's emission redshift; and (3) "associated complex systems," which are clustered with $z_{\mathrm{abs}} \simeq z_{\mathrm{em}}$. The optically thick systems are those systems which are optically thick at the Lyman limit; they are usually identified on the basis of $\mathrm{Mg}$ II absorption at low to moderate redshifts or Lyman limit absorption at high redshift (cf. Bergeron and Stansinska 1986; Tytler et al. 1987; LTW; Wolfe 1987; Lanzetta 1988). The optically thin systems are optically thin at the Lyman limit; they generally lack low ions and are selected on the basis of C IV absorption at moderate to high redshifts (cf. Young, Sargent, and Boksenberg 1982). The associated complex systems are usually (but not always) optically thin at the Lyman limit; they normally display very high ions (e.g., $\mathrm{N} \mathrm{v}$ and $\mathrm{C}$ IV), and the velocity extent of their absorption is often large (e.g., $\Delta v_{\text {metals }}>500 \mathrm{~km} \mathrm{~s}^{-1}$ ). Associated complex systems can be identified on the basis of a survey for $\mathrm{C}$ IV absorption near the emission redshift, and they are preferentially found in radio-loud QSOs (cf. Anderson et al. 1987; Foltz et al. 1988). Aside from the fact that the damped Ly $\alpha$ systems exhibit large neutral hydrogen column densities, which is an obvious selection bias, we discuss below how the properties of the damped Ly $\alpha$ systems differ from the properties of other QSO absorption-line systems.

i) Selection Bias in Other QSO Absorption-Line Surveys

While surveys for damped Ly $\alpha$ will select a population of absorbers which are not biased with respect to their metal line properties, all other types of QSO absorption-line surveys will introduce biases which are a function of the metal abundances, the effective velocity dispersion and velocity extent of the metal lines, and the ionization. For example, spectroscopic surveys based on $\mathrm{C}$ IV absorption alone and designed to detect lines with rest-frame equivalent widths $W \geq 0.3 \AA$ identify complete samples of optically thin and associated complex systems only down to a specified high-ion equivalent width threshold. Systems with appreciably lower or higher ionization states, as well as systems with very low effective velocity dispersions, could be missed in such surveys. If selection of the damped Ly $\alpha$ systems were based on $\mathrm{C}$ IV absorption alone, about half of the systems would be missed, despite the fact that damped Ly $\alpha$ systems are large column density systems which may have some low ions with $W>1 \AA$. Damped Ly $\alpha$ systems appear to be a special subset of the optically thick metal line systems which can be identified on the basis of having Lyman limit or possibly $\mathrm{Mg}$ II absorption. However, using Lyman limit or $\mathrm{Mg}$ II absorption as a selection criterion is not a very efficient means of identifying damped Ly $\alpha$ systems. From existing surveys we indirectly deduce that only $\sim 20 \%$ of the optically thick systems, identified on the basis of having Lyman limit or $\mathrm{Mg}$ II absorption, exhibit damped Ly $\alpha$ absorption with $N(\mathrm{H} \mathrm{I}) \geq 2 \times 10^{20} \mathrm{~cm}^{-2}$. Finally, since the number of damped Ly $\alpha$ systems studied so far is small, finding all damped Ly $\alpha$ systems in an intermediate-resolution survey for $\mathrm{Mg}$ II is not guaranteed. Damped systems in which $W(\mathrm{Mg}$ II $)<0.3 \AA$ could conceivably exist as a result of some combination of abundance and effective velocity dispersion effects. In fact, the $z_{\text {abs }}=1.7890$ system toward Q1136 +112 may be just such a case.

ii) Equivalent Width

In damped Ly $\alpha$ systems the total equivalent width of the $\mathrm{Mg}$ II low-ion absorption is generally larger than the total equivalent width of the $C$ IV high-ion absorption (Fig. 14). Thus, the damped Ly $\alpha$ systems bear little resemblance to the optically thin and associated complex systems in terms of the equivalent widths of the low and high-ions (cf. Wolfe 1983; Danly, Blades, and Norman 1987). However, in absolute terms the strength of the low- and high-ion absorption in damped Ly $\alpha$ systems is similar to that observed in a sample of Mg II selected systems (compare Fig. 14 with Fig. 10 of LTW). On the basis of absorption equivalent widths of the low and high-ions, the damped Ly $\alpha$ systems appear indistinguishable from the optically thick population of metal line systems. The similarity between the damped Ly $\alpha$ and $\mathrm{Mg}$ II systems can be further established by the resemblance between the frequency distribution of $W(\mathrm{Mg}$ II) and velocity dispersion, $\sigma$ (Paper II).

iii) Kinematics of Low and High Ions and Tendency to Cluster

Some fraction of all four types of metal line systems exhibit evidence for multicomponent structure on relatively small velocity scales (e.g., $\Delta v_{\text {metals }}<500 \mathrm{~km} \mathrm{~s}^{-1}$ ). Whether or not multicomponent structure is observed often depends on the 
resolution. Generally, $\Delta v_{\text {metals }}$ seldom exceeds $\sim 500 \mathrm{~km} \mathrm{~s}^{-1}$ for the damped Ly $\alpha$ systems, the optically thick systems, or the optically thin systems. Improvements in resolution may eventually allow one to use $\Delta v_{\text {metals }}$ or the absorption profiles themselves to discriminate statistically between these three types of systems. Of course, based on the results of $\S$ III, in some instances very small values of the effective velocity dispersion, i.e., $\sigma<10 \mathrm{~km} \mathrm{~s}^{-1}$, are derived from simple curve-of-growth analysis applied to the metal lines of damped Ly $\alpha$ systems. The associated complex systems often have $\Delta v_{\text {metals }}$ exceeding $\sim 500 \mathrm{~km} \mathrm{~s}^{-1}$; this distinguishes the associated complex systems from the other three types.

In addition to searching for small-scale velocity structure in metal line systems, large-scale velocity structure (or "clustering") can also be studied. By definition, the associated complex systems are clearly clustered near the QSO emission redshift, while the other three types of metal line systems are not. The presence of large-scale velocity structure or clustering may also manifest itself by the presence of additional individual absorption systems within several thousand kilometers per second of the metal line system under study. A recent investigation (Sargent, Boksenberg, and Steidel 1988) of the two-point correlation function for metal line systems shows that no evidence for clustering on scales in excess of $\sim 600 \mathrm{~km} \mathrm{~s}^{-1}$ exists, but this study excludes optically thick systems with damped Ly $\alpha$ absorption for all practical purposes. On the other hand, some associated complex systems clearly show evidence for clustering of systems on scales in excess of $\sim 600 \mathrm{~km} \mathrm{~s}^{-1}$; whether this is due to outflow from the QSO or a rich cluster in which the QSO is embedded is unclear. Of the 18 damped Ly $\alpha$ systems identified in the Lick survey (see $\S$ III $b, c)$, data exist in 14 of the cases to make a preliminary search for both optically thick and optically thin metal line systems within several thousand kilometers per second of the damped redshift. Five of these 14 damped systems clearly show other systems within several thousand kilometers per second of the damped redshift: Q0100+130 has two other systems (SYBT; Black, Chaffee, and Foltz 1987), Q0458-020 has one other system (Paper IV), Q1151 + 068 has one other system (this study), Q1244+347 has three other systems (Paper IV), and Q2136 + 141 has one other system (Paper IV). In particular, if a redshift window within $6000 \mathrm{~km} \mathrm{~s}^{-1}$ of either side of the damped Ly $\alpha$ redshift is chosen, this covers a path length corresponding to $\Delta z \simeq 1.8$ at a mean redshift $z \simeq 2.2$. Surveys have shown that one would expect to find about two metal line systems within this redshift window, and yet eight metal line systems are found. This may suggest that metal line systems tend to cluster around the damped Ly $\alpha$ system redshifts. Given this estimate, the evidence for an enhanced number of absorbers in the vicinity of a damped $\mathrm{Ly} \alpha$ absorber is significant at the $4 \sigma$ level, but in any case it should be verified, since the data base is not homogeneous (i.e., the estimate that only two systems should have been found is uncertain) and small numbers are involved (i.e., the uncertainty in the number of detected systems, 8 , is large). If clustering is present, the cosmological scale corresponds to supercluster size, i.e., several tens of megaparsecs. Absorption-line observations of the Tololo pair $1037-2704$ and $1038-2712$ have also been interpreted in terms of superclustering on these scales (Sargent and Steidel 1987). In the case of the Tololo pair, the incidence and complexity of the absorption is much higher, and the fact that the $z_{\text {abs }}=2.1378$ system in Tol 1037-2704 may be a damped Ly $\alpha$ system with $N(\mathrm{H} \mathrm{I}) \simeq 10^{20} \mathrm{~cm}^{-2}$ is interesting.
Other results worthy of note include the fact that the damped Ly $\alpha$ absorber seen in the spectrum of Q1331+170 has an optically thick system displaced $\sim 760 \mathrm{~km} \mathrm{~s}^{-1}$ from the damped Ly $\alpha$ redshift (cf. LTW; Chaffee, Black, and Foltz 1988). In addition, the damped Ly $\alpha$ absorber seen in the spectrum of PKS $1157+014$ has two broad optically thin systems displaced from it, one $\sim 2500 \mathrm{~km} \mathrm{~s}^{-1}$ to the shortward side and the other $\sim 2900 \mathrm{~km} \mathrm{~s}^{-1}$ to the longward side (Briggs, Turnshek, and Wolfe 1984); both of these optically thin systems have a large velocity extent in the $\mathrm{C}$ IV metal lines with $\Delta v_{\text {metals }} \simeq 1300$ and $3000 \mathrm{~km} \mathrm{~s}^{-1}$, respectively. These optically thin systems in PKS 1157+014 may belong to the associated complex class of systems that frequently occur in radio-loud QSOs.

\section{iv) Incidence of $21 \mathrm{~cm}$ Absorption}

The only QSO metal line systems exhibiting $21 \mathrm{~cm}$ absorption are the damped Ly $\alpha$ systems (cf. Briggs 1988). On physical grounds, the optically thin metal line systems and associated complex systems have little chance of causing $21 \mathrm{~cm}$ absorption. Moreover, in a sample of 18 metal line systems selected on the basis of $\mathrm{Mg}$ II absorption (i.e., optically thick systems), no $21 \mathrm{~cm}$ absorption lines were found in cases where the Ly $\alpha$ absorption was not damped (Briggs and Wolfe 1983). Thus, 21 $\mathrm{cm}$ absorption is a distinguishing characteristic of the damped Ly $\alpha$ systems. In a few cases $21 \mathrm{~cm}$ absorption has been looked for in damped Ly $\alpha$ systems and has not been found; whether this is due to the physical size of the background radio source or the physical conditions in the absorber is unclear.

v) Interpretation

That the metal line properties of the damped Ly $\alpha$ systems are indistinguishable from the $\mathrm{Mg}$ II producing gas indicates that most damped Ly $\alpha$ systems include a metal-enriched component of turbulent gas which is optically thick at the Lyman limit. However, the latter is not the highly opaque component of $\mathrm{H}$ I which gives rise to both damped Ly $\alpha$ and $21 \mathrm{~cm}$ absorption. What distinguishes the damped Ly $\alpha$ systems from the majority of $\mathrm{Mg}$ II absorbers is the presence of this highly opaque component of $\mathrm{H}$ I. Studies in the $21 \mathrm{~cm}$ absorption line show this component to be quiescent in comparison with the $\mathbf{M g}$ II gas, with $\sigma\left(\mathrm{H}_{\mathrm{I}}\right)<10 \mathrm{~km} \mathrm{~s}^{-1}$ as compared with $\langle\sigma(\mathrm{Mg} \mathrm{II})\rangle \sim 50 \mathrm{~km} \mathrm{~s}^{-1}$ for the $\mathrm{Mg}$ II systems. Our curve-ofgrowth studies of the low-ion gas in the $z_{\mathrm{abs}}=2.7957$ absorber toward Q1337+113 and the $z_{\text {abs }}=1.7737$ absorber toward $\mathrm{Q} 1151+068$ show that $\sigma<10 \mathrm{~km} \mathrm{~s}^{-1}$, indicating that the quiescent component can be detected in the metal lines when the turbulent component is absent. In the two cases noted above, where metal lines of the quiescent component were detected, high-ion absorption was not detected. Therefore, in common with the turbulent component, the ionization state of the quiescent component is low. This is hardly surprising in view of the large Lyman limit optical depth, $\tau_{L L}>10^{3}$, of the quiescent component.

Because most QSO spectroscopy is of intermediate resolution, we are currently limited in the extent to which we can study the multicomponent structure of most damped Ly $\alpha$ systems. From intermediate-resolution optical spectroscopy alone, we can usually only infer that a damped Ly $\alpha$ system is consistent with having at least one absorption component that is characterized by (1) large neutral hydrogen column density, e.g., $N(\mathrm{H} \mathrm{I}) \geq 10^{20} \mathrm{~cm}^{-2}$, and (2) low ions which dominate over high ions. The problem of having limited spectral resolution at optical wavelengths can be overcome when the background 
QSO is a radio source by taking advantage of what can be learned from high-resolution $21 \mathrm{~cm}$ absorption-line studies of damped Ly $\alpha$ systems (cf. Paper II and Briggs 1988). At the same time, however, the velocity dispersions of the high column density neutral hydrogen clouds can be very small, i.e., $\sigma \leq 5 \mathrm{~km} \mathrm{~s}^{-1}$, and metal lines arising from a single cloud with a very small $\sigma$ will not be easy to detect in intermediateresolution spectra which have normal signal-to-noise ratios. Much can undoubtedly be learned through future highresolution studies of damped Ly $\alpha$ systems, especially if the turbulent gas component is not present.

At present, the $z_{\text {abs }}=2.040$ absorber toward PKS 0458-020 represents the only case for direct evidence of a quiescent disk with galactic dimensions (Wolfe et al. 1985; Briggs et al. 1989; Paper IV). However, several lines of evidence indicate that all damped Ly $\alpha$ systems arise in quiescent disks. First, aside from the system seen in the spectrum of PKS $0458-020$, the quiescent component has been detected optically in two cases studied in this paper $\left(z_{\text {abs }}=1.7737\right.$ in $\mathrm{Q} 1151+123$ and $z_{\mathrm{abs}}=2.7957$ in Q1337+113) and in two other cases (aside from PKS 0458-020) studied in Paper IV. Therefore, the quiescent component has been detected in five of the damped Ly $\alpha$ systems identified in the Lick survey. Second, $21 \mathrm{~cm}$ studies of damped Ly $\alpha$ systems not included in the Lick survey and of systems at redshifts too low for Ly $\alpha$ to be observed from the ground show the presence of five additional quiescent systems (Wolfe 1987; Briggs 1988); the $21 \mathrm{~cm}$ absorption systems toward AO $0235+164$ and 3C 196 have transverse dimension exceeding $\sim 10 h^{-1} \mathrm{kpc}$ (Foltz, Chaffee, and Wolfe 1988). A reasonable conclusion is that all damped Ly $\alpha$ systems arise in disklike structures. Paper II argues that the quiescent components found in the Lick survey are drawn from a unique population of disklike structures that are the high-redshift progenitors of the stellar disks in present-day galaxies. It then follows that the turbulent gas component sometimes associated with damped Ly $\alpha$ is caused by the halo which envelops the disk. This view is consistent with the gas recently found to be associated with the outskirts of a significant population of galaxies with $z \sim 0.5$ (Bergeron 1988).

\section{c) Further Comments on Interpretation and Abundance Determinations}

As we have emphasized throughout, because low column density gas with high effective velocity dispersion creates lines with larger equivalent widths than does high column density gas with low effective velocity dispersion, the column densities derived from the curve-of-growth studies and presented in Tables 11-15 should probably be interpreted as strictly lower limits to the actual values. In particular, if a damped Ly $\alpha$ system exhibits a turbulent component, the turbulent component will dominate the equivalent widths, despite the fact that the quiescent component contains most of the gas. Thus, in most instances, the column densities inferred from a curveof-growth analysis of QSO absorption-line systems correspond solely to a turbulent component. From the work of Jenkins (1986), one might argue that, when the turbulent component dominates the equivalent widths of the metal lines, the deduced column densities can be used to give a relative determination of metal abundances for the turbulent component only; no information on the absolute abundances for the turbulent component can be inferred, because of the presence of the strong damped Ly $\alpha$ line arising in the quiescent component. On the other hand, two damped Ly $\alpha$ systems with low effective veloc- ity dispersion have been found in this study, indicating that they contain only a quiescent component: the $z_{\text {abs }}=1.7737$ system toward Q1151+068 and the $z_{\text {abs }}=2.7957$ system toward Q1337+113. In this section these two systems are reconsidered and discussed. The Q1136+112 $z_{\text {abs }}=1.7890$ system and three of the systems summarized in $\S$ III $c$, for which previous authors derived abundances, are also discussed.

$$
\text { i) The } Q 1136+112 z_{\text {abs }}=1.7890 \text { System }
$$

Our spectrum of Q1136+112 showed no evidence for C II $\lambda 1334, \mathrm{O}_{\text {I }} \lambda 1302$, or Si II $\lambda 1304$ at $z_{\text {abs }}=1.7890$ down to a $3 \sigma$ rest equivalent width limit of $\sim 0.2 \AA$ or better. However, these lines were predicted to occur in the Ly $\alpha$ forest, and a value for the effective velocity dispersion of the system could not be derived. If the $3 \sigma$ detection upper limits given in Table 8 are adopted and if the lines are assumed to lie on the damping part of the curve of growth, the maximum column densities for each of these three ions are $\log N\left(\mathrm{C}^{+}\right)<17.7, \log N\left(\mathrm{O}^{\circ}\right)<17.5$, and $\log N\left(\mathrm{Si}^{+}\right)<17.5$. For the only detected line, Fe II $22382, \log$ $N\left(\mathrm{Fe}^{+}\right)=13.0$ is found when the line is assumed to lie on the linear part of the curve of growth, while $\log N\left(\mathrm{Fe}^{+}\right)=15.8$ is found when the line is assumed to lie on the damping part of the curve of growth. These upper limits correspond to upper limits on the metal-to-hydrogen abundance ratios, since the gas is in a low-ionization state because of its optical thickness and $\log N\left(\mathrm{H}^{0}\right)=20.0$ is known. With the exception of the poor limit placed on $\mathrm{Si}^{+}$, the limits are consistent with abundances which are 1-10 times solar values. Analysis of better observations of this system might yield more interesting results, if only a quiescent component is found to be present.

$$
\text { ii) The } Q 1151+068 z_{\text {abs }}=1.7737 \text { System }
$$

The curve-of-growth analysis based on the Fe II and Mg II metal lines indicates a very small value $\sigma \simeq 9 \mathrm{~km} \mathrm{~s}^{-1}$ for the effective velocity dispersion. Thus, only a quiescent component appears to be present in this system, giving rise to both the damped Ly $\alpha$ line and low-ion metal lines. If a neutral hydrogen column density of $N(\mathrm{H} \mathrm{I}) \simeq 1.8 \times 10^{21}$ is adopted (see Table 9, but also see $\S$ III $b[$ iv $]$ ), the derived best-fit column densities from the curve of growth (Table 12) indicate $\log \left[\mathrm{C}^{+} /\right.$ $\left.\mathrm{H}^{0}\right] \simeq-5.6, \log \left[\mathrm{O}^{0} / \mathrm{H}^{0}\right] \simeq-3.1, \log \left[\mathrm{Al}^{+} / \mathrm{H}^{0}\right] \simeq-5.6, \log$ $\left[\mathrm{Mg}^{+} / \mathrm{H}^{0}\right] \simeq-6.1, \log \left[\mathrm{Si}^{+} / \mathrm{H}^{0}\right] \simeq-5.8$, and $\log \left[\mathrm{Fe}^{+} /\right.$ $\left.\mathrm{H}^{0}\right] \simeq-5.9$. These values range from $5 \times 10^{-3}$ to 1 times solar values, assuming that all of the hydrogen and oxygen are neutral and that all of the carbon, aluminum, magnesium, silicon, and iron are singly ionized. In the interstellar medium of the Galaxy, some elements such as aluminum appear to be depleted relative to solar values by factors of up to 100 or more. The fact that aluminum in this system is inferred to have an abundance which is approximately the solar value may indicate that significant depletion has not occurred and that dust has not formed.

$$
\text { iii) The } Q 1337+113 z_{\text {abs }}=2.7957 \text { System }
$$

The curve-of-growth analysis of the low-ion Si II and Fe II metal lines indicates a very small value for the effective velocity dispersion, $\sigma \simeq 8 \mathrm{~km} \mathrm{~s}^{-1}$, Again, only a quiescent component appears to be present in this system, giving rise to both damped Ly $\alpha$ and the low-ion metal lines. Following the same procedure as above, the best-fit results reported in Table 13 indicate that the abundances of carbon, oxygen, aluminum, silicon, and iron lie in the range $4 \times 10^{-3}$ to 0.5 times solar values. See Lanzetta, Wolfe, and Turnshek (1989) for more details, including a discussion of upper limits on the $\mathrm{H}_{2}$ and dust content of the absorbing gas. 
iv) Other Damped Lyman-Alpha Systems in the Lick Survey with Reported Abundance Determinations

The $z_{\text {abs }}=2.309$ system toward $Q 0100+130 .-\mathrm{Q} 0100+130$ is the well-known QSO PHL 957. The damped Ly $\alpha$ absorption system in this object was discussed in some detail by Black, Chaffee and Foltz (1987). A line list was published by SYBT. Other published work includes Lowrance et al. (1972), Morton and Morton (1972), and Beaver et al. (1972). $N(\mathrm{H} \mathrm{I})=2.5 \times 10^{21} \mathrm{~cm}^{-2}$ is inferred from excellent fits to the $\operatorname{Ly} \alpha, \operatorname{Ly} \beta$, and $\operatorname{Ly} \gamma$ profiles. The system is characterized by relatively strong unresolved low-ion metal line absorption and weak high-ion metal line absorption. The curve-of-growth study by Black, Chaffee, and Foltz (1987) indicates that $\sigma \simeq 18$ $\mathrm{km} \mathrm{s}^{-1}$ for the low-ion gas. The extremely high signal-to-noise ratio spectrum of this bright object has allowed these same authors to explore upper limits on the strength of neutral carbon absorption, $\mathrm{H}_{2}$ absorption, and $\mathrm{CO}$ absorption. No absorption from these species is detected. The model which Black, Chaffee, and Foltz (1987) present suggests that the carbon-to-hydrogen abundance ratio is $\sim 10^{-2}$ times the solar value, that the $\mathrm{CO}$-to-hydrogen ratio is $<10^{-1}$ times the Galactic value, and that the $\mathrm{H}_{2}$ to hydrogen ratio is $<8 \times 10^{-6}$ times the Galactic value.

The $z_{\text {abs }}=1.920$ system toward Q2206-199N.-This system has been discussed by Robertson, Shaver, and Carswell (1983). They derive $N(\mathrm{H} \mathrm{I}) \simeq 7 \times 10^{20} \mathrm{~cm}^{-2}$ from the damped Ly $\alpha$ profile. The system is characterized by relatively strong lowand high-ion metal line absorption. They report that the derived value of the velocity dispersion from a curve-of-growth analysis is very uncertain, with $\sigma \simeq 80 \mathrm{~km} \mathrm{~s}^{-1}$ being the best estimate. Based on this determination, they suggest that abundances ranging from $10^{-1}$ to 1 times the solar value are plausible.

The $z_{\text {abs }}=2.077$ system toward $Q 2206-199 N$.- This system has also been discussed by Robertson, Shaver, and Carswell (1983). The system is characterized by relatively strong low-ion and weak high-ion metal line absorption. They derive $N(\mathrm{H} \mathrm{I}) \simeq 5 \times 10^{20} \mathrm{~cm}^{-2}$ from the damped Ly $\alpha$ profile and deduce $\sigma \geq 20 \mathrm{~km} \mathrm{~s}^{-1}$ from a curve-of-growth analysis. They suggest that the abundances in this system may be $<10^{-2}$ times the solar value.

Limitations on the abundance determinations.-In the cases of the Q0100+130 $z_{\text {abs }}=2.309$ system, the Q2206-199N $z_{\text {abs }}=$ 1.920 system, and the Q2206-199N $z_{\text {abs }}=2.077$ system, the previous observers derived effective velocity dispersions of $\sigma \simeq 18 \mathrm{~km} \mathrm{~s}^{-1}, \sigma \simeq 80 \mathrm{~km} \mathrm{~s}^{-1}$, and $\sigma \geq 20 \mathrm{~km} \mathrm{~s}^{-1}$, respectively, from the low-ion metal lines. Adopting our interpretation, all of these metal line systems must originate in a turbulent component, since the derived velocity dispersion is relatively large. Thus, we believe that the abundance determinations in these systems are misleading, since the bulk of the neutral hydrogen is likely to arise in a quiescent component with much smaller velocity dispersion. Any low-ion metal lines that would arise in the quiescent component are impossible to assess at intermediate resolution, owing to the masking caused by the presence of the turbulent component.

\section{v) Summary}

In the two cases where only a quiescent component was identified (i.e., the Q1151+068 results in Table 12 and the Q1337+113 results in Table 13), the curves of growth indicate that abundances lie in the range $4 \times 10^{-3}$ to 1 times solar values, with the actual determinations being strictly lower limits, provided that our identification and measurement of metal line equivalent widths (sometimes in the presence of Ly $\alpha$ forest absorption) are substantially correct. In these systems aluminum appears not to be significantly depleted, which would indicate that significant amounts of dust have not formed. The errors are difficult to evaluate, but realistically they are probably about a factor of 10 . However, we must stress that even in the two cases where only a quiescent component appears to be present, a single-component model for the absorption may not be the appropriate assumption. These damped Ly $\alpha$ systems could still have more than one component. For example, a component with $\alpha \simeq 10 \mathrm{~km} \mathrm{~s}^{-1}$ may give rise to the metal lines, and a second component with $\sigma$ significantly less than $10 \mathrm{~km} \mathrm{~s}^{-1}$ may give rise to the bulk of the large column of neutral hydrogen. If this occurs, evidence for abundances which are less than their true values will erroneously be deduced. Tighter constraints require considerably higher resolution observations. Future studies of abundances in damped $\mathrm{Ly} \alpha$ systems should concentrate on those systems which apparently show no sign of a turbulent component. Finally, as the work of Jenkins (1986) suggests, the relative metal abundances of the turbulent components seen in Q0836+113 (Table 11), Q1347+112 (Table 14), and Q2348-011 (Table 15) may be roughly correct.

\section{d) The Incidence of Damped Lyman-Alpha Absorption in the Lick Survey}

In Table 3 and Figure 4 of Paper I we reported on the status of intermediate-resolution spectroscopy of the 47 candidates for damped Ly $\alpha$ absorption in the 68 QSOs that were included in the Lick survey. In a similar manner, we present here a current report on the nature of those 47 absorption features. The 47 candidate absorption features appeared in 30 of the QSO spectra. Based on the $3 \AA$ resolution spectroscopy obtained at Lick Observatory (Smith, Cohen, and Bradley 1986), the $1.5 \AA$ resolution spectroscopy of PKS 2206-199N (Robertson, Shaver, and Carswell 1983), the $1 \AA$ resolution spectroscopy of PHL 957 (Black, Chaffee, and Foltz 1987), the 1-2.4 $\AA$ resolution spectroscopy reported on here, the $1-2.4 \AA$ resolution spectroscopy of Paper IV, and the $1 \AA$ spectroscopy at near-ultraviolet wavelengths of Q1337+113 (Lanzetta, Wolfe, and Turnshek 1989), we believe that at least 18 of the 47 strong absorption features reported in Paper I are damped Ly $\alpha$. These 18 damped Ly $\alpha$ lines occur in 14 QSO spectra. Of the remaining 29 absorption features, we believe that one feature is due to a single $\operatorname{Ly} \beta$ absorption line and 13 are not primarily due to a single damped Ly $\alpha$ absorption line. This leaves 15 strong absorption features whose nature is undetermined. A detailed summary of the status for each of the 47 features is reported in Table 17. In Figure 15 the rest-frame absorption equivalent width (as measured from the lowresolution data of Paper I) is presented for the 47 features, which indicates whether or not the features are damped. Since the features with an undetermined nature generally have the lowest equivalent widths and since inspection of the lowresolution spectroscopy shows them to be the poorest candidate damped Ly $\alpha$ lines, it may be unlikely that any of the remaining 15 strong absorption features are primarily due to a single damped Ly $\alpha$ absorption line with $N(\mathrm{H} \mathrm{I}) \geq 2 \times 10^{20}$ $\mathrm{cm}^{-2}$. As discussed in Papers I and II, this column density is the physically relevant lower limit adopted in the analysis presented in Paper II. Based on the analysis of individual systems (see $\S$ III), two of the 18 features which we believe are damped 
TABLE 17

Status of the Candidates for Damped Lyman-Alpha Absorption

\begin{tabular}{|c|c|c|c|c|}
\hline QSO & $\stackrel{\lambda}{(\AA)^{\mathrm{a}}}$ & $\begin{array}{c}E \\
(\AA)^{\mathrm{b}}\end{array}$ & $\begin{array}{l}W \\
(\AA)^{\mathrm{c}}\end{array}$ & Comments \\
\hline \multirow[t]{2}{*}{ 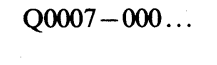 } & 3376 & 15.2 & 5.5 & Nature undetermined \\
\hline & 3662 & 18.0 & 6.0 & Nature undetermined \\
\hline Q0037-018...... & 3602 & 15.5 & 5.2 & Nature undetermined \\
\hline $\mathrm{Q} 0045-013 \ldots \ldots$ & 3491 & 14.5 & 5.1 & Nature undetermined \\
\hline $\mathrm{Q} 0049+007 \ldots \ldots$ & 3544 & 22.6 & 7.8 & Nature undetermined \\
\hline \multirow[t]{3}{*}{$\mathrm{Q} 0100+130 \ldots \ldots$} & 3396 & 15.8 & 5.7 & $\begin{array}{l}\text { Ly } \beta \text { associated with } \\
\text { damped Ly } \alpha^{\mathrm{d}}\end{array}$ \\
\hline & 4033 & 106.5 & 32.1 & Damped Ly $\alpha^{\mathrm{d}}$ \\
\hline & 4332 & 23.3 & 6.5 & Not damped $\operatorname{Ly} \alpha^{\mathrm{d}}$ \\
\hline $\mathrm{Q} 0149+336 \ldots \ldots$ & 3813 & 33.4 & 10.7 & Damped Ly $\alpha^{\mathrm{e}}$ \\
\hline $\mathrm{Q} 0457+024 \ldots \ldots$ & 3391 & 31.1 & 11.2 & Not damped $\operatorname{Ly} \alpha^{f}$ \\
\hline \multirow{2}{*}{$\mathrm{Q} 0458-020 \ldots \ldots$} & 3712 & 90.2 & 29.5 & Damped Ly $\alpha^{8, \hat{h}}$ \\
\hline & 3921 & 19.3 & 6.0 & $\begin{array}{l}\text { C IV and } L y \alpha \text { forest } \\
\text { absorption }^{g, h}\end{array}$ \\
\hline Q0552+398.. & 3283 & 15.9 & 5.9 & Nature undetermined \\
\hline $\mathrm{Q} 0836+113 \ldots \ldots$ & 4216 & 41.1 & 11.9 & Damped $L y \alpha^{i}$ \\
\hline 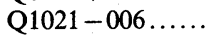 & 3527 & 16.8 & 5.8 & Nature undetermined \\
\hline $\mathrm{Q} 1123+264 \ldots \ldots$ & 3550 & 23.4 & 8.0 & Not damped $\operatorname{Ly} \alpha^{\mathrm{f}}$ \\
\hline Q1128+105 $\ldots \ldots$ & 4191 & 21.4 & 6.2 & Not damped $L y \alpha^{j}$ \\
\hline $\mathrm{Q} 1136+122 \ldots$ & 3395 & 29.5 & 10.6 & Damped Ly $\alpha^{\mathrm{k}}$ \\
\hline \multirow[t]{2}{*}{ Q1151+068 ..... } & 3374 & 112.9 & 40.7 & Damped Ly $\alpha^{k}$ \\
\hline & 3420 & 21.5 & 7.6 & Not damped $\operatorname{Ly} \alpha^{\mathrm{k}}$ \\
\hline $\mathrm{Q} 1215+333 \ldots \ldots$ & 3648 & 62.8 & 20.9 & Damped Ly $\alpha^{\mathrm{e}}$ \\
\hline $\mathrm{Q} 1244+347 \ldots \ldots$ & 3473 & 20.3 & 7.1 & Damped Ly $\alpha^{\mathrm{e}}$ \\
\hline \multirow{3}{*}{$\mathrm{Q} 1337+113 \ldots \ldots$} & 3821 & 23.6 & 7.5 & Not damped $L y \alpha^{1}$ \\
\hline & 4268 & 24.1 & 6.9 & Probably damped Ly $\alpha^{1}$ \\
\hline & 4614 & 60.2 & 15.9 & Damped Ly $\alpha^{\mathrm{i}}$ \\
\hline 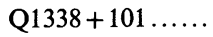 & 3450 & 17.4 & 6.1 & Nature undetermined \\
\hline \multirow{2}{*}{$\mathrm{Q} 1347+112 \ldots \ldots$} & 3716 & 30.2 & 9.9 & Nature undetermined \\
\hline & 4226 & 30.1 & 8.7 & Damped Ly $\alpha^{i}$ \\
\hline \multirow[t]{2}{*}{$\mathrm{Q} 1352+108 \ldots \ldots$} & 3928 & 22.8 & 7.1 & Nature undetermined \\
\hline & 4194 & 24.6 & 7.1 & Not damped $\operatorname{Ly} \alpha^{\mathrm{f}}$ \\
\hline \multirow[t]{3}{*}{$\mathrm{Q} 1402+044 \ldots \ldots$} & 4238 & 33.2 & 9.5 & Nature undetermined \\
\hline & 4484 & 30.9 & 8.4 & Nature undetermined \\
\hline & 4515 & 22.3 & 6.0 & Nature undetermined \\
\hline $\mathrm{Q} 1429+118 \ldots \ldots$ & 4412 & 18.9 & 5.2 & Not damped $\operatorname{Ly} \alpha^{\mathrm{f}}$ \\
\hline \multirow[t]{2}{*}{$\mathrm{Q} 1451+123 \ldots \ldots$} & 4228 & 37.6 & 10.8 & Nature undetermined \\
\hline & 5074 & 21.1 & 5.1 & Nature undetermined \\
\hline $\mathrm{Q} 1503+118 \ldots \ldots$ & 3807 & 18.6 & 5.9 & Not damped $\operatorname{Ly} \alpha^{\mathrm{j}}$ \\
\hline \multirow{2}{*}{$\mathrm{Q} 2136+141 \ldots \ldots$} & 3792 & 16.1 & 5.2 & Damped $\mathrm{Ly} \alpha^{\mathrm{g}, \mathrm{m}}$ \\
\hline & 3811 & 18.2 & 5.8 & Not damped $\operatorname{Ly} \alpha^{\mathrm{g}, \mathrm{m}}$ \\
\hline \multirow{2}{*}{ Q2206-199N ... } & 3559 & 39.1 & 13.4 & Damped Ly $\alpha^{\mathrm{n}}$ \\
\hline & 3742 & 26.7 & 8.7 & Damped Ly $\alpha^{\mathrm{n}}$ \\
\hline \multirow{2}{*}{$\mathrm{Q} 2256+017 \ldots \ldots$} & 3495 & 15.2 & 5.3 & Not damped $L y \alpha^{j}$ \\
\hline & 3553 & 24.8 & 8.5 & Not damped $\operatorname{Ly} \alpha^{j}$ \\
\hline \multirow[t]{2}{*}{$\mathrm{Q} 2348-011 \ldots \ldots$} & 4167 & 24.3 & 7.1 & Damped Ly $\alpha^{\mathrm{i}}$ \\
\hline & 4384 & 70.1 & 19.4 & Damped Ly $\alpha^{\mathrm{i}}$ \\
\hline \multirow{2}{*}{$\mathrm{Q} 2359-022 \ldots \ldots$} & 3764 & 43.8 & 14.1 & Damped Ly $\alpha^{\mathrm{e}}$ \\
\hline & 3830 & 23.0 & 7.3 & Damped Ly $\alpha^{\mathrm{e}}$ \\
\hline
\end{tabular}

NoTE.-The following systems, discussed by Smith, Cohen, and Bradley 1986, were not among the 47 strong candidate damped Ly $\alpha$ features presented in Paper I: (1) Q0049+014 $z_{\text {abs }}=1.830$ system, (2) Q0149+336 $z_{\text {abs }}=2.134$ system, (3) Q1406+123 $z_{\text {abs }}=1.835$ system, (4) Q1406+123 $z_{\text {abs }}=2.252$ system, (5) Q1503+118 $z$ abs $=2.598$ system, and (6) Q2136+141 $z_{\text {abs }}=1.823$ system. Hunstead 1988 has studied the spectrum of Q1406+123 and believes that the $z_{\text {abs }}=1.835$ system is not damped and that the $z_{\text {abs }}=2.252$ system is damped with $N(\mathrm{H} \mathrm{I}) \simeq 3 \times 10^{20} \mathrm{~cm}^{-2}$. In keeping with the rigorous criteria set forth in Paper I, we have not included any of these systems in the $N(\mathrm{H}$ 1) $\geq 2 \times 10^{20} \mathrm{~cm}^{-2}$ damped Ly $\alpha$ sample discussed in $\S$ IV $d$.

a Wavelength centroid of the line profile from the low-resolution data in Paper I.

${ }^{b}$ Observed equivalent width from the low-resolution data in Paper I.

c Rest-frame equivalent width, assuming a Ly $\alpha$ identification, from the lowresolution data in Paper I.

d Based on the $1 \AA$ resolution MMT observations of Black, Chaffee, and Foltz 1987.

e Based on the $1 \AA$ resolution MMT observations presented in Paper IV.

f The low-resolution observations presented in Paper I are sufficient to rule out a damping profile.

${ }^{8}$ Based on the $2 \AA$ resolution MMT observations presented in Paper IV. in fact have neutral hydrogen column densities less than this lower limit. Therefore, keeping in mind that 15 candidate systems have not been observed, we report that the Lick survey has thus far resulted in the discovery of 16 damped Ly $\alpha$ systems with column densities $N(\mathrm{H}$ I $) \geq 2 \times 10^{20}$ over a cosmological path length $\Delta z=56$, yielding $d N /$ $d z \geq 0.29 \pm 0.07$ at a mean redshift $z=2.24$. Note that the status of observations reported in Table 17 supersedes the results on the incidence of damped Ly $\alpha$ absorption reported in Paper I and in Smith, Cohen, and Bradley (1986). Some of the systems which Smith, Cohen, and Bradley (1986) suggest contain damped Ly $\alpha$ have not been confirmed (see Table 17). At the same time, Smith, Cohen, and Bradley (1986) did report the existence of a $z_{\text {abs }}=2.252$ system in Q1406+123 with $N(\mathrm{H} \mathrm{I})=3 \times 10^{20} \mathrm{~cm}^{-2}$ which Hunstead (1988) confirms. This system was not reported as a candidate damped Ly $\alpha$ line in Paper I. Thus, the value we report for $d N / d z$ may be regarded as a lower limit.

\section{SUMMARY}

A summary of the main conclusions from this work are as follows:

1. Intermediate-resolution spectroscopy has been used to conclude that at least 18 of the 47 strong Ly $\alpha$ absorption features $(>40 \%)$ identified in the Lick low-resolution spectroscopic survey are damped. Sixteen of these features can be fitted with damping profiles corresponding to $N(\mathrm{H} \mathrm{I}) \geq 2 \times 10^{20} \mathrm{~cm}^{-2}$, while the remaining two correspond to lower column density. These systems arise over a cosmological path length $\Delta z=56$ at a mean redshift $z=2.24$. The intermediate-resolution spectroscopy and analysis pertaining to seven of these sytems were presented, and results for the remaining 11 systems were summarized. The 16 systems noted here can be considered the minimum number of damped Ly $\alpha$ systems with $N\left(\mathrm{H} \mathrm{I}^{\mathrm{I}}\right) \geq 2 \times 10^{20} \mathrm{~cm}^{-2}$ in the Lick survey sample. This yields $d N / d z \geq 0.29 \pm 0.07$ for such systems.

2. The properties of the damped Ly $\alpha$ systems were compared with the properties of other QSO metal absorption-line systems, and the following results were found: (1) about half of the damped Ly $\alpha$ systems would have been missed if they were searched for on the basis of C IV absorption alone; (2) the damped Ly $\alpha$ systems may be viewed as a small subset of the "optically thick metal line systems" which are identified on the basis of Mg II or Lyman limit absorption; (3) a tendency for metal line systems to cluster around the damped Ly $\alpha$ system redshift on a scale within $\sim 6000 \mathrm{~km} \mathrm{~s}^{-1}$ was found; and (4) the damped Ly $\alpha$ systems are the only QSO absorption-line systems which can show $21 \mathrm{~cm}$ absorption.

3. Based largely on $21 \mathrm{~cm}$ absorption-line studies of a few of the damped Ly $\alpha$ systems as well as results from our curves of growth, we concluded that a typical system contains a quiescent component and often contains a turbulent component. The

\footnotetext{
${ }^{\text {h }}$ See the observations of Wolfe et al. 1985 as well as the $2 \AA$ resolution observations presented in Paper IV.

i Based on the $2 \AA$ resolution MMT observations presented here.

${ }^{\mathrm{j}}$ Damped Ly $\alpha$ is ruled out on the basis of unpublished $3 \AA$ resolution observations by Smith and Cohen.

k Based on the $1 \AA$ resolution MMT observations presented here.

${ }^{1}$ Based on the $1 \AA$ resolution MMT observations presented in Lanzetta, Wolfe, and Turnshek 1989.

m In Paper I, Q2136 + 141 has reported features at 3792 and $3811 \AA$; these are probably due to a single absorption feature.

${ }^{n}$ Based on the $1.5 \AA$ resolution AAT observations of Robertson, Shaver, and Carswell 1983.
} 


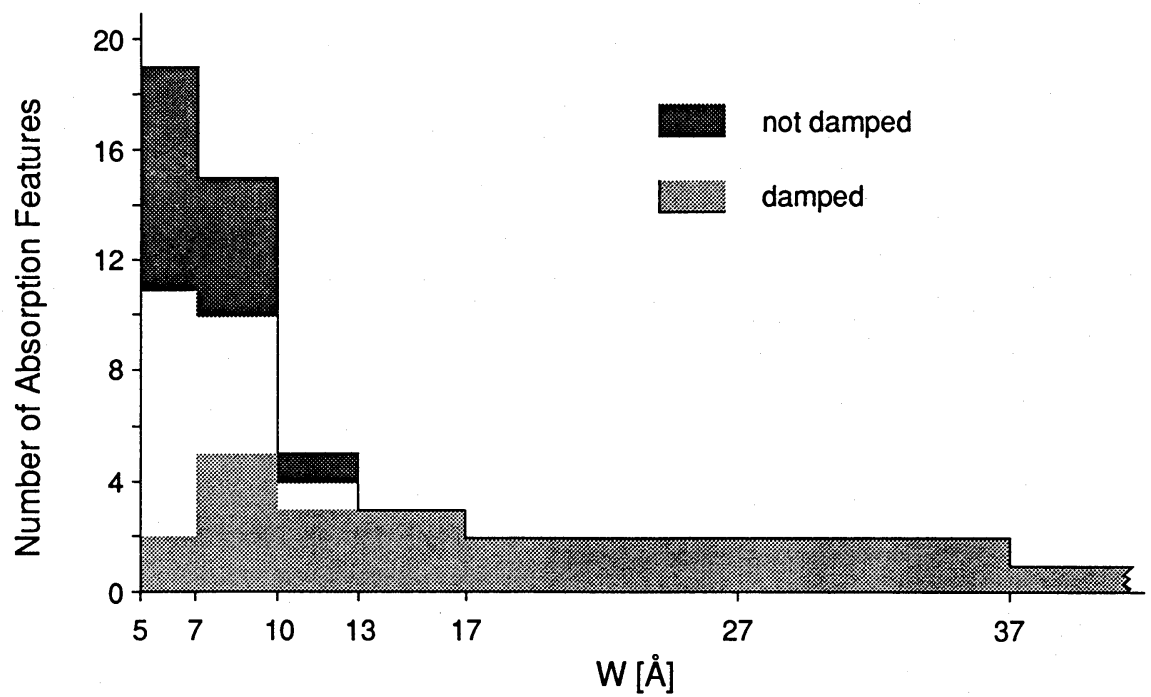

Fig. 15.-Illustration showing the nature of the 47 strong absorption features identified in the earlier Lick low-resolution spectroscopic survey. Note that the bins have unequal widths.

quiescent component contains most of the $\mathrm{H} \mathrm{I}$, which gives rise to damped $\mathrm{Ly} \alpha$ and $21 \mathrm{~cm}$ absorption, has an effective velocity dispersion $\sigma<10 \mathrm{~km} \mathrm{~s}^{-1}$, and generally contains only low ionization states of abundant elements. The turbulent component, if present, contains a small fraction of the total $\mathrm{H} \mathrm{I}$, has an effective velocity dispersion $\sigma>20 \mathrm{~km} \mathrm{~s}^{-1}$, and spans a velocity interval of $\Delta v_{\text {metals }} \geq 150 \mathrm{~km} \mathrm{~s}^{-1}$, and, while it may contain both low and high ions, the low-ion equivalent widths are usually larger. About half the time the high ions are not detected. The quiescent components are likely drawn from a unique population of disklike structures that are the highredshift progenitors of galactic disks. The turbulent components may reside in halos which envelop the disks.

4. Evidence was presented that abundances in two quiescent components of damped Ly $\alpha$ systems lie in the range $4 \times 10^{-3}$ to 1 times the solar value, with these determinations taken to be lower limits, provided that our metal line identifications and measurements are substantially correct (sometimes in the presence of $\mathrm{Ly} \alpha$ forest absorption). These results were derived from curve-of-growth analysis under the assumption that only a single quiescent component exists in each system; therefore, higher resolution observations should be made to confirm these results.

We are indebted to the former provost of the University of Pittsburgh, Dr. Roger Benjamin, for supplying crucial support which financed some of the observations made at the MMT. We also thank the competent staffs at the MMT Observatory and CTIO for helping us obtain these data, and Dr. Jill Bechtold for valuable discussions. D. A. T. and A. M. W. wish to thank Ray Weymann for the hospitality afforded them while on leave at Mount Wilson and Las Campanas Observatories, where some of this work was completed. H. E. S. and A. M. W. also thank Dr. Weymann for some assistance at the MMT early on in this project. This work was partially supported by NSF grant AST 8715070 to A. M. W. and F. H. B.; H. E. S., and R. D. C. were partially supported by NSF grant AST 8414658 .

\section{REFERENCES}

Anderson, S. A., Weymann, R. J., Foltz, C. B., and Chaffee, F. H., Jr. 1987, A.J., 94, 278.

Beaver, E. A., Burbidge, E. M., McIlwain, C. E., Epps, H. W., and Strittmatter, P. A. 1972, Ap. J., 178, 95

Bechtold, J. 1988, private communication

Bechtold, J., Green, R. F., and York, D. G. 1987, Ap. J., 312, 50.

Bergeron, J. 1988, in OSO Absorption Lines: Probing the Universe, ed. J. C. Blades, D. A. Turnshek, and C. A. Norman (Cambridge: Cambridge University Press), p. 127

Bergeron, J., and Stasinska, G. 1986, Astr. Ap., 169, 1.

Black, J. H., Chaffee, F. H., Jr., and Foltz, C. B. 1987, Ap. J., 317, 442.

Briggs, F. 1988, in OSO Absorption Lines: Probing the Universe, ed. J. C. Blades, D. A. Turnshek, and C. A. Norman (Cambridge: Cambridge University Press), p. 275

Briggs, F. H., Turnshek, D. A., and Wolfe, A. M. 1984, Ap. J., 287, 549.

Briggs, F. H., and Wolfe, A. M. 1983, Ap. J., 268, 76

Briggs, F. H., Wolfe, A. M., Liszt, H., Davis, M. M., and Turner, K. L. 1989, Ap. J., 341, 650 .

Chaffee, F. H., Jr., Black, J. H., and Foltz, C. B. 1988, Ap. J., 335, 584

Danly, L., Blades, J. C., and Norman, C. A. 1987, in QSO Absorption Lines: Probing the Universe, Poster Papers, ed. J. C. Blades, C. A. Norman, and D. A. Turnshek (Baltimore: STSCI) p. 88.

Foltz, C. B., Chaffee, F. H., Jr., and Weymann, R. J. 1986, A.J., 92, 247

Foltz, C. B., Chaffee, F. H., Jr., Weymann, R. J., and Anderson, S. A. 1988, in QSO Absorption Lines: Probing the Universe, ed. J. C. Blades, D. A. Turnshek, and C. A. Norman (Cambridge: Cambridge University Press), p. 53.
Foltz, C. B., Chaffee, F. H., Jr., and Wolfe, A. M. 1988, Ap. J., 335, 35.

Horne, K. 1986, Pub. A.S.P., 98, 609.

Hunstead, R. W. 1988, private communication.

Jenkins, E. 1986, Ap. J., 304, 739.

Lanzetta, K. M. 1988, Ap. J., 332, 96.

Lanzetta, K. M., Turnshek, D. A., and Wolfe, A. M. 1987, Ap. J., 322, 739 (LTW).

Lanzetta, K. M., Wolfe, A. M., and Turnshek, D. A. 1989, Ap.J., in press.

Lanzetta, K. M., et al. 1988, private communication.

Lowrance, J. L., Morton, D. C., Zucchino, P., Oke, J. B., and Schmidt, M. 1972 Ap.J., 171, 233 .

Morton, D. C., and Morton, W. A. 1972, Ap. J. 174, 237.

Morton, D. C., York, D. G., and Jenkins, E. 1988, Ap. J. Suppl., 68, 449.

Robertson, J.' G., Shaver, P. A., and Carswell, R. F. 1983, in Quasars and Gravitational Lenses, ed, J. P. Swings (Liège: University of Liège), p. 602

Sargent, W. L. W., Boksenberg, A. and Steidel, C. 1988, Ap. J. Suppl., 68, 539.

Sargent, W. L. W., and Steidel, C. 1987, Ap.J., 322, 142

Sargent, W. L. W., Young, P. J., Boksenberg, A., and Tytler, D. 1980, Ap. J. Suppl., 42, 41 (SYBT)

Smith, H. E., Cohen, R. D., and Bradley, S. E. 1986, Ap. J., 310, 583

Tytler, D. 1987, A.J., 321, 69.

Tytler, D., Boksenberg, A., Sargent, W. L. W., Young, P. and Kunth, D. 1987, Ap. J.S Suppl., 64, 667.

Wolfe, A. M. 1983, Ap. J. (Letters), 268, L1.

. 1987, in Proc. Arecibo Upgrading Workshop, ed. J. Taylor and M. M. Davis (Arecibo: NAIC), p. 81. 
Wolfe, A. M. 1988, in OSO Absorption Lines: Probing the Universe, ed. J. C. Blades, D. A. Turnshek, and C. A. Norman (Cambridge: Cambridge University Press), p. 297.

Wolfe, A. M., Briggs, F. H., Turnshek, D. A., Davis, M. M., Smith, H. E., and Cohen, R. D. 1985, Ap. J.(Letters), 294, L67.

In Wolfe, A. M., Turnshek, D. A., and Lanzetta, K. M. 1989, in preparation II. Wolfe, A. M.,

Frank H. Briggs, David A. Turnshek, and Arthur M. Wolfe: Department of Physics and Astronomy, University of Pittsburgh, Pittsburgh, PA 15260

Ross D. Cohen and Harding E. Smith: Center for Astrophysics and Space Sciences, C-011, University of California, San Diego, La Jolla, CA 92093

CRAIG B. FolTz: MMT Observatory, University of Arizona, Tucson, AZ 85721

KenNeTH M. LANZETTA: Institute of Astronomy, Cambridge University, Cambridge CB3 0HA, United Kingdom

BeLINDA J. Wilkes: Smithsonian Astrophysical Observatory, 60 Garden Street, Cambridge, MA 02138 